\title{
Long-baseline neutrino oscillation physics potential of the DUNE experiment
}

\author{
DUNE Collaboration
}

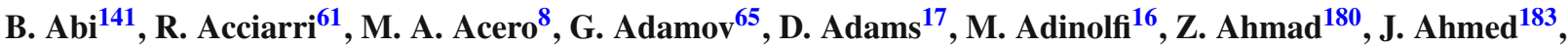
T. Alion ${ }^{169}$, S. Alonso Monsalve ${ }^{21}$, C. Alt ${ }^{53}$, J. Anderson ${ }^{4}$, C. Andreopoulos ${ }^{158,118}$, M. P. Andrews ${ }^{61}$, F. Andrianala ${ }^{2}$, S. Andringa ${ }^{113,114}$, A. Ankowski ${ }^{159}$, M. Antonova ${ }^{77}$, S. Antusch ${ }^{10}$, A. Aranda-Fernandez ${ }^{39}$, A. Ariga ${ }^{11}$, L. O. Arnold ${ }^{42}$, M. A. Arroyave ${ }^{52}$, J. Asaadi ${ }^{173}$, A. Aurisano ${ }^{37}$, V. Aushev ${ }^{112}$, D. Autiero ${ }^{89}$, F. Azfar ${ }^{141}$, H. Back ${ }^{142}$, J. J. Back ${ }^{183}$, C. Backhouse ${ }^{178}$, P. Baesso ${ }^{16}$, L. Bagby ${ }^{61}$, R. Bajou ${ }^{144}$, S. Balasubramanian ${ }^{187}$, P. Baldi ${ }^{26}$, B. Bambah ${ }^{75}$, F. Bara0 ${ }^{91,113,114}$, G. Barenboim ${ }^{77}$, G. J. Barker ${ }^{183}$, W. Barkhouse ${ }^{135}$, C. Barnes ${ }^{125}$, G. Barr ${ }^{141}$, J. Barranco Monarca ${ }^{70}$, N. Barros ${ }^{55,113,114}$, J. L. Barrow ${ }^{61,171}$, A. Bashyal ${ }^{140}$, V. Basque ${ }^{123}$, F. Bay ${ }^{134}$, J. L. Bazo Alba ${ }^{151}$, J. F. Beacom ${ }^{139}$, E. Bechetoille ${ }^{89}$, B. Behera ${ }^{41}$, L. Bellantoni ${ }^{61}$, G. Bellettini ${ }^{149}$, V. Bellini ${ }^{33,79}$, O. Beltramello ${ }^{21}$, D. Belver ${ }^{22}$, N. Benekos ${ }^{21}$, F. Bento Neves ${ }^{113,114}$, J. Berger ${ }^{150}$, S. Berkman ${ }^{61}$, P. Bernardini ${ }^{81,161}$, R. M. Berner ${ }^{11}$, H. Berns ${ }^{25}$, S. Bertolucci ${ }^{14,78}$, M. Betancourt ${ }^{61}$, Y. Bezawada ${ }^{25}$, M. Bhattacharjee ${ }^{95}$, B. Bhuyan ${ }^{95}$, S. Biagi ${ }^{87}$, J. Bian ${ }^{26}$, M. Biassoni ${ }^{82}$, K. Biery ${ }^{61}$, B. Bilki ${ }^{12,99}$, M. Bishai ${ }^{17}$, A. Bitadze ${ }^{123}$, A. Blake ${ }^{116}$, B. Blanco Siffert ${ }^{60}$, F. D. M. Blaszczyk ${ }^{61}$, G. C. Blazey ${ }^{136}$, E. Blucher ${ }^{35}$, J. Boissevain ${ }^{119}$, S. Bolognesi ${ }^{20}$, T. Bolton ${ }^{109}$, M. Bonesini ${ }^{82,127}$, M. Bongrand ${ }^{115}$, F. Bonini ${ }^{17}$, A. Booth ${ }^{169}$, C. Booth ${ }^{163}$, S. Bordoni ${ }^{21}$, A. Borkum ${ }^{169}$,

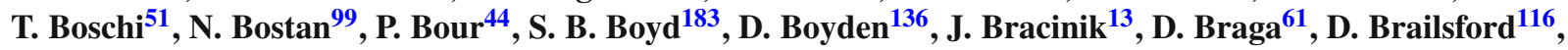
A. Brandt ${ }^{173}$, J. Bremer ${ }^{21}$, C. Brew ${ }^{158}$, E. Brianne ${ }^{123}$, S. J. Brice ${ }^{61}$, C. Brizzolari ${ }^{82,127}$, C. Bromberg ${ }^{126}$, G. Brooijmans ${ }^{42}$, J. Brooke ${ }^{16}$, A. Bross ${ }^{61}$, G. Brunetti ${ }^{85}$, N. Buchanan ${ }^{41}$, H. Budd ${ }^{155}$, D. Caiulo ${ }^{89}$, P. Calafiura ${ }^{117}$, J. Calcutt ${ }^{126}$, M. Calin ${ }^{18}$, S. Calvez ${ }^{41}$, E. Calvo ${ }^{22}$, L. Camilleri ${ }^{42}$, A. Caminata ${ }^{80}$, M. Campanelli ${ }^{178}$, D. Caratelli ${ }^{61}$,

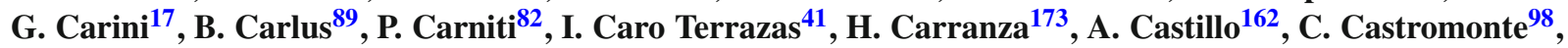
C. Cattadori $^{82}$, F. Cavalier ${ }^{115}$, F. Cavanna ${ }^{61}$, S. Centro ${ }^{143}$, G. Cerati ${ }^{61}$, A. Cervelli ${ }^{78}$, A. Cervera Villanueva ${ }^{77}$, M. Chalifour ${ }^{21}$, C. Chang ${ }^{28}$, E. Chardonnet ${ }^{144}$, A. Chatterjee ${ }^{150}$, S. Chattopadhyay ${ }^{180}$, J. Chaves ${ }^{146}$, H. Chen ${ }^{17}$, M. Chen ${ }^{26}$, Y. Chen ${ }^{11}$, D. Cherdack ${ }^{74}$, C. Chi ${ }^{42}$, S. Childress ${ }^{61}$, A. Chiriacescu ${ }^{18}$, K. Cho ${ }^{107}$, S. Choubey ${ }^{71}$, A. Christensen ${ }^{41}$, D. Christian ${ }^{61}$, G. Christodoulou ${ }^{21}$, E. Church ${ }^{142}$, P. Clarke ${ }^{54}$, T. E. Coan ${ }^{167}$, A. G. Cocco ${ }^{84}$, J. A. B. Coelho ${ }^{115}$, E. Conley ${ }^{50}$, J. M. Conrad ${ }^{124}$, M. Convery ${ }^{159}$, L. Corwin ${ }^{164}$, P. Cotte $^{20}$, L. Cremaldi ${ }^{131}$, L. Cremonesi ${ }^{178}$, J. I. Crespo-Anadón ${ }^{22}$, E. Cristaldo ${ }^{6}$, R. Cross ${ }^{116}$, C. Cuesta $^{22}$, Y. Cui ${ }^{28}$, D. Cussans ${ }^{16}$, M. Dabrowski ${ }^{17}$, H. da Motta $^{19}$, L. Da Silva Peres ${ }^{60}$, C. David ${ }^{61,189}$, Q. David ${ }^{89}$, G. S. Davies ${ }^{131}$, S. Davini ${ }^{80}$, J. Dawson ${ }^{144}$, K. De ${ }^{173}$, R. M. De Almeida ${ }^{63}$, P. Debbins ${ }^{99}$, I. De Bonis ${ }^{47}$, M. P. Decowski ${ }^{134,1}$, A. de Gouvêa ${ }^{137}$, P. C. De Holanda ${ }^{32}$, I. L. De Icaza Astiz ${ }^{169}$, A. Deisting ${ }^{156}$, P. De Jong ${ }^{134,1}$, A. Delbart ${ }^{20}$, D. Delepine ${ }^{70}$, M. Delgado ${ }^{3}$, A. Dell'Acqua ${ }^{21}$, P. De Lurgio ${ }^{4}$, J. R. T. de Mello Neto ${ }^{60}$, D. M. DeMuth ${ }^{179}$, S. Dennis ${ }^{31}$, C. Densham ${ }^{158}$, G. Deptuch ${ }^{61}$, A. De Roeck ${ }^{21}$, V. De Romeri ${ }^{77}$, J. J. De Vries ${ }^{31}$, R. Dharmapalan ${ }^{73}$, M. Dias ${ }^{177}$, F. Diaz ${ }^{151}$, J. S. Díaz ${ }^{97}$, S. Di Domizio ${ }^{64,80}$, L. Di Giulio ${ }^{21}$, P. Ding ${ }^{61}$, L. Di Noto ${ }^{64,80}$, C. Distefano ${ }^{87}$, R. Diurba ${ }^{130}$, M. Diwan ${ }^{17}$, Z. Djurcic ${ }^{4}$, N. Dokania ${ }^{168}$, M. J. Dolinski ${ }^{49}$, L. Domine ${ }^{159}$, D. Douglas ${ }^{126}$, F. Drielsma ${ }^{159}$, D. Duchesneau ${ }^{47}$, K. Duffy ${ }^{61}$, P. Dunne ${ }^{94}$, T. Durkin ${ }^{158}$, H. Duyang ${ }^{166}$, O. Dvornikov ${ }^{73}$, D. A. Dwyer ${ }^{117}$, A. S. Dyshkant ${ }^{136}$, M. Eads $^{136}$, D. Edmunds ${ }^{126}$, J. Eisch ${ }^{100}$, S. Emery ${ }^{20}$, A. Ereditato ${ }^{11}$, C. O. Escobar ${ }^{61}$, L. Escudero Sanchez ${ }^{31}$, J. J. Evans ${ }^{123}$, E. Ewart ${ }^{97}$, A. C. Ezeribe ${ }^{163}$, K. Fahey ${ }^{61}$, A. Falcone ${ }^{82,127}$, C. Farnese ${ }^{143}$, Y. Farzan ${ }^{\text {90 }}$, J. Felix ${ }^{70}$, E. Fernandez-Martinez ${ }^{122}$, P. Fernandez Menendez ${ }^{77}$, F. Ferraro ${ }^{64,80}$, L. Fields ${ }^{61}$, A. Filkins ${ }^{185}$, F. Filthaut ${ }^{134,154}$, R. S. Fitzpatrick ${ }^{125}$, W. Flanagan ${ }^{46}$, B. Fleming ${ }^{187}$, R. Flight ${ }^{155}$, J. Fowler ${ }^{50}$, W. Fox ${ }^{97}$, J. Franc ${ }^{44}$, K. Francis ${ }^{136}$,

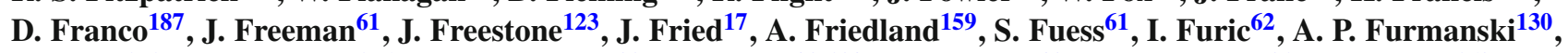
A. Gago ${ }^{151}$, H. Gallagher ${ }^{176}$, A. Gallego-Ros ${ }^{22}$, N. Gallice ${ }^{83,128}$, V. Galymov ${ }^{89}$, E. Gamberini ${ }^{21}$, T. Gamble ${ }^{163}$, R. Gandhi ${ }^{71}$, R. Gandrajula126, S. Gao ${ }^{17}$, D. Garcia-Gamez ${ }^{68}$, M. Á. García-Peris ${ }^{77}$, S. Gardiner ${ }^{61}$, D. Gastler ${ }^{15}$, G. Ge ${ }^{42}$, B. Gelli ${ }^{32}$, A. Gendotti ${ }^{53}$, S. Gent ${ }^{165}$, Z. Ghorbani-Moghaddam ${ }^{80}$, D. Gibin ${ }^{143}$, I. Gil-Botella ${ }^{22}$, C. Girerd ${ }^{89}$, A. K. Giri ${ }^{96}$, D. Gnani ${ }^{117}$, O. Gogota ${ }^{112}$, M. Gold ${ }^{132}$, S. Gollapinni ${ }^{119}$, K. Gollwitzer ${ }^{61}$, R. A. Gomes ${ }^{57}$, L. V. Gomez Bermeo ${ }^{162}$, L. S. Gomez Fajardo ${ }^{162}$, F. Gonnella ${ }^{13}$, J. A. Gonzalez-Cuevas ${ }^{6}$, M. C. Goodman ${ }^{4}$, O. Goodwin ${ }^{123}$, S. Goswami ${ }^{148}$, C. Gotti ${ }^{82}$, E. Goudzovski ${ }^{13}$, C. Grace ${ }^{117}$, M. Graham ${ }^{159}$, E. Gramellini ${ }^{187}$, 
R. Gran ${ }^{129}$, E. Granados ${ }^{70}$, A. Grant ${ }^{48}$, C. Grant ${ }^{15}$, D. Gratieri ${ }^{63}$, P. Green ${ }^{123}$, S. Green ${ }^{31}$, L. Greenler ${ }^{186}$, M. Greenwood ${ }^{140}$, J. Greer ${ }^{16}$, W. C. Griffith ${ }^{169}$, M. Groh ${ }^{97}$, J. Grudzinski ${ }^{4}$, K. Grzelak ${ }^{182}$, W. Gu ${ }^{17}$, V. Guarino ${ }^{4}$, R. Guenette ${ }^{72}$, A. Guglielmi ${ }^{85}$, B. Guo ${ }^{166}$, K. K. Guthikonda ${ }^{108}$, R. Gutierrez ${ }^{3}$, P. Guzowski ${ }^{123}$, M. M. Guzzo ${ }^{32}$,

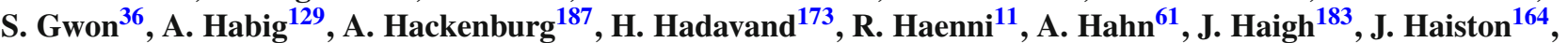
T. Hamernik ${ }^{61}$, P. Hamilton ${ }^{94}$, J. Han ${ }^{150}$, K. Harder ${ }^{158}$, D. A. Harris ${ }^{61,189}$, J. Hartnell ${ }^{169}$, T. Hasegawa ${ }^{106}$, R. Hatcher ${ }^{61}$, E. Hazen ${ }^{15}$, A. Heavey ${ }^{61}$, K. M. Heeger ${ }^{187}$, J. Heise ${ }^{160}$, K. Hennessy ${ }^{118}$, S. Henry ${ }^{155}$, M. A. Hernandez Morquecho ${ }^{70}$, K. Herner ${ }^{61}$, L. Hertel ${ }^{26}$, A. S. Hesam ${ }^{21}$, J. Hewes ${ }^{37}$, A. Higuera ${ }^{74}$, T. Hill ${ }^{92}$, S. J. Hillier ${ }^{13}$, A. Himmel ${ }^{61}$, J. Hoff ${ }^{61}$, C. Hohl ${ }^{10}$, A. Holin ${ }^{178}$, E. Hoppe ${ }^{142}$, G. A. Horton-Smith ${ }^{109}$, M. Hostert ${ }^{51}$, A. Hourlier ${ }^{124}$, B. Howard ${ }^{61}$, R. Howell ${ }^{155}$, J. Huang ${ }^{174}$, J. Huang ${ }^{25}$, J. Hugon ${ }^{120}$, G. Iles ${ }^{94}$, N. Ilic ${ }^{175}$, A. M. Iliescu ${ }^{78}$, R. Illingworth ${ }^{61}$, A. Ioannisian ${ }^{188}$, R. Itay ${ }^{159}$, A. Izmaylov ${ }^{77}$, E. James ${ }^{61}$, B. Jargowsky ${ }^{26}$, F. Jediny ${ }^{44}$, C. Jesùs-Valls ${ }^{76}$, X. Ji ${ }^{17}$, L. Jiang ${ }^{181}$, S. Jiménez ${ }^{22}$, A. Jipa ${ }^{18}$, A. Joglekar ${ }^{28}$, C. Johnson ${ }^{41}$, R. Johnson ${ }^{37}$, B. Jones ${ }^{173}$, S. Jones ${ }^{178}$, C. K. Jung ${ }^{168}$, T. Junk ${ }^{61}$, Y. Jwa ${ }^{42}$, M. Kabirnezhad ${ }^{141}$, A. Kaboth ${ }^{158}$, I. Kadenko ${ }^{112}$, F. Kamiya ${ }^{59}$, G. Karagiorgi ${ }^{42}$, A. Karcher ${ }^{117}$, M. Karolak ${ }^{20}$, Y. Karyotakis ${ }^{47}$, S. Kasai ${ }^{111}$, S. P. Kasetti ${ }^{120}$, L. Kashur ${ }^{41}$, N. Kazaryan ${ }^{188}$, E. Kearns ${ }^{15}$, P. Keener ${ }^{146}$, K. J. Kelly ${ }^{61}$, E. Kemp ${ }^{32}$, W. Ketchum ${ }^{61}$, S. H. Kettell ${ }^{17}$, M. Khabibullin ${ }^{88}$, A. Khotjantsev ${ }^{88}$, A. Khvedelidze ${ }^{65}$, D. Kim $^{21}$, B. King ${ }^{61}$, B. Kirby ${ }^{17}$, M. Kirby ${ }^{61}$, J. Klein ${ }^{146}$, K. Koehler ${ }^{186}$, L. W. Koerner ${ }^{74}$, S. Kohn ${ }^{24,117}$, P. P. Koller ${ }^{11}$, M. Kordosky ${ }^{185}$, T. Kosc ${ }^{89}$, U. Kose ${ }^{21}$, V. A. Kostelecký ${ }^{97}$, K. Kothekar ${ }^{16}$, F. Krennrich ${ }^{100}$, I. Kreslo ${ }^{11}$, Y. Kudenko ${ }^{88}$, V. A. Kudryavtsev ${ }^{163}$, S. Kulagin ${ }^{88}$, J. Kumar ${ }^{73}$, R. Kumar ${ }^{153}$, C. Kuruppu ${ }^{166}$, V. Kus ${ }^{44}$, T. Kutter ${ }^{120}$, A. Lambert ${ }^{117}$, K. Lande ${ }^{146}$, C. E. Lane ${ }^{49}$, K. Lang ${ }^{174}$, T. Langford ${ }^{187}$, P. Lasorak ${ }^{169}$, D. Last ${ }^{146}$, C. Lastoria $^{22}$, A. Laundrie ${ }^{186}$, A. Lawrence ${ }^{117}$, I. Lazanu ${ }^{18}$, R. LaZur ${ }^{41}$, T. Le ${ }^{176}$, J. Learned ${ }^{73}$, P. LeBrun ${ }^{89}$, G. Lehmann Miotto ${ }^{21}$, R. Lehnert ${ }^{97}$, M. A. Leigui de Oliveira ${ }^{59}$,

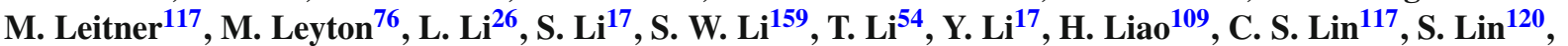
A. Lister ${ }^{186}$, B. R. Littlejohn ${ }^{93}$, J. Liu' ${ }^{26}$, S. Lockwitz ${ }^{61}$, T. Loew ${ }^{117}$, M. Lokajicek ${ }^{43}$, I. Lomidze ${ }^{65}$, K. Long ${ }^{94}$, K. Loo ${ }^{105}$, D. Lorca ${ }^{11}$, T. Lord ${ }^{183}$, J. M. LoSecco ${ }^{138}$, W. C. Louis ${ }^{119}$, K. B. Luk ${ }^{24,117}$, X. Luo ${ }^{29}$, N. Lurkin ${ }^{13}$, T. Lux $^{76}$, V. P. Luzio ${ }^{59}$, D. MacFarland ${ }^{159}$, A. A. Machado ${ }^{32}$, P. Machado61, C. T. Macias ${ }^{97}$, J. R. Macier ${ }^{61}$, A. Maddalena ${ }^{67}$, P. Madigan ${ }^{24,117}$, S. Magill ${ }^{4}$, K. Mahn ${ }^{126}$, A. Maio ${ }^{55,113,114}$, J. A. Maloney ${ }^{45}$, G. Mandrioli ${ }^{78}$, J. Maneira ${ }^{55,113,114}$, L. Manenti ${ }^{178}$, S. Manly ${ }^{155}$, A. Mann ${ }^{176}$, K. Manolopoulos ${ }^{158}$, M. Manrique Plata ${ }^{97}$, A. Marchionni ${ }^{61}$, W. Marciano ${ }^{17}$, D. Marfatia ${ }^{73}$, C. Mariani ${ }^{181}$, J. Maricic ${ }^{73}$, F. Marinho ${ }^{58}$, A. D. Marino ${ }^{40}$, M. Marshak ${ }^{130}$, C. Marshall ${ }^{117, a}$, J. Marshall ${ }^{183}$, J. Marteau ${ }^{89}$, J. Martin-Albo ${ }^{77}$, N. Martinez ${ }^{109}$, D. A. Martinez Caicedo ${ }^{164}$, S. Martynenko ${ }^{168}$, K. Mason $^{176}$, A. Mastbaum ${ }^{157}$, M. Masud ${ }^{77}$, S. Matsuno ${ }^{73}$, J. Matthews ${ }^{120}$, C. Mauger ${ }^{146}$, N. Mauri ${ }^{14,78}$, K. Mavrokoridis ${ }^{118}$, R. Mazza $^{82}$, A. Mazzacane ${ }^{61}$, E. Mazzucato ${ }^{20}$, E. McCluskey $^{61}$, N. McConkey ${ }^{123}$, K. S. McFarland ${ }^{155}$, C. McGrew ${ }^{168}$, A. McNab ${ }^{123}$, A. Mefodiev ${ }^{88}$, P. Mehta ${ }^{103}$, P. Melas ${ }^{7}$, M. Mellinato ${ }^{82,127}$, O. Mena ${ }^{77}$, S. Menary ${ }^{189}$, H. Mendez ${ }^{152}$, A. Menegolli ${ }^{86,145}$, G. Meng ${ }^{85}$, M. D. Messier ${ }^{97}$, W. Metcalf ${ }^{120}$, M. Mewes ${ }^{97}$, H. Meyer ${ }^{184}$, T. Miao ${ }^{61}$, G. Michna ${ }^{165}$, T. Miedema ${ }^{134,154}$, J. Migenda ${ }^{163}$, R. Milincic ${ }^{73}$, W. Miller ${ }^{130}$, J. Mills ${ }^{176}$, C. Milne ${ }^{92}$, O. Mineev ${ }^{88}$, O. G. Miranda ${ }^{38}$, S. Miryala ${ }^{17}$, C. S. Mishra ${ }^{61}$, S. R. Mishra ${ }^{166}$, A. Mislivec ${ }^{130}$, D. Mladenov ${ }^{21}$, I. Mocioiu' ${ }^{147}$, K. Moffat ${ }^{51}$, N. Moggi ${ }^{14,78}$, R. Mohanta ${ }^{75}$, T. A. Mohayai ${ }^{61}$, N. Mokhov ${ }^{61}$, J. Molina ${ }^{6}$, L. Molina Bueno ${ }^{53}$, A. Montanari ${ }^{78}$, C. Montanari ${ }^{86,145}$, D. Montanari ${ }^{61}$, L. M. Montano Zetina ${ }^{38}$, J. Moon ${ }^{124}$, M. Mooney ${ }^{41}$, A. Moor ${ }^{31}$, D. Moreno ${ }^{3}$, B. Morgan ${ }^{183}$, C. Morris $^{74}$, C. Mossey ${ }^{61}$, E. Motuk ${ }^{178}$, C. A. Moura ${ }^{59}$, J. Mousseau ${ }^{125}$, W. Mu' ${ }^{61}$, L. Mualem ${ }^{30}$, J. Mueller ${ }^{41}$, M. Muether ${ }^{184}$, S. Mufson ${ }^{97}$, F. Muheim ${ }^{54}$, A. Muir ${ }^{48}$, M. Mulhearn ${ }^{25}$, H. Muramatsu ${ }^{130}$, S. Murphy ${ }^{53}$, J. Musser ${ }^{97}$, J. Nachtman ${ }^{99}$, S. Nagu ${ }^{121}$, M. Nalbandyan ${ }^{188}$, R. Nandakumar ${ }^{158}$, D. Naples ${ }^{150}$, S. Narita ${ }^{101}$, D. Navas-Nicolás ${ }^{22}$, N. Nayak ${ }^{26}$, M. Nebot-Guinot ${ }^{54}$, L. Necib ${ }^{30}$, K. Negishi ${ }^{101}$, J. K. Nelson ${ }^{185}$, J. Nesbit ${ }^{186}$, M. Nessi ${ }^{21}$, D. Newbold ${ }^{158}$, M. Newcomer ${ }^{146}$, D. Newhart ${ }^{61}$, R. Nichol ${ }^{178}$, E. Niner ${ }^{61}$, K. Nishimura ${ }^{73}$, A. Norman ${ }^{61}$, A. Norrick ${ }^{61}$, R. Northrop ${ }^{35}$, P. Novella ${ }^{77}$, J. A. Nowak ${ }^{116}$, M. Oberling ${ }^{4}$, A. Olivares Del Campo ${ }^{51}$, A. Olivier ${ }^{155}$, Y. Onel ${ }^{99}$,

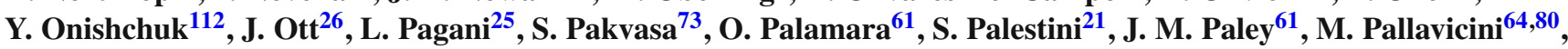
C. Palomares ${ }^{22}$, E. Pantic ${ }^{25}$, V. Paolone ${ }^{150}$, V. Papadimitriou ${ }^{61}$, R. Papaleo ${ }^{87}$, A. Papanestis ${ }^{158}$, S. Paramesvaran ${ }^{16}$, S. Parke ${ }^{61}$, Z. Parsa ${ }^{17}$, M. Parvu ${ }^{18}$, S. Pascoli ${ }^{51}$, L. Pasqualini ${ }^{14,78}$, J. Pasternak ${ }^{94}$, J. Pater ${ }^{123}$, C. Patrick ${ }^{178}$, L. Patrizii ${ }^{78}$, R. B. Patterson ${ }^{30}$, S. J. Patton ${ }^{117}$, T. Patzak ${ }^{144}$, A. Paudel ${ }^{109}$, B. Paulos ${ }^{186}$, L. Paulucci ${ }^{59}$, Z. Pavlovic ${ }^{61}$, G. Pawloski ${ }^{130}$, D. Payne ${ }^{118}$, V. Pec ${ }^{163}$, S. J. M. Peeters ${ }^{169}$, Y. Penichot ${ }^{20}$, E. Pennacchio ${ }^{89}$, A. Penzo ${ }^{99}$, O. L. G. Peres ${ }^{32}$, J. Perry ${ }^{54}$, D. Pershey ${ }^{50}$, G. Pessina ${ }^{82}$, G. Petrillo ${ }^{159}$, C. Petta ${ }^{33,79}$, R. Petti ${ }^{166}$, F. Piastra ${ }^{11}$, L. Pickering ${ }^{126}$, F. Pietropaolo ${ }^{21,85}$, J. Pillow ${ }^{183}$, J. Pinzino ${ }^{175}$, R. Plunkett ${ }^{61}$, R. Poling ${ }^{130}$, X. Pons ${ }^{21}$, N. Poonthottathil ${ }^{100}$, S. Pordes ${ }^{61}$, M. Potekhin ${ }^{17}$, R. Potenza ${ }^{33,79}$, B. V. K. S. Potukuchi ${ }^{102}$, J. Pozimski ${ }^{94}$, M. Pozzato ${ }^{14,78}$, S. Prakash ${ }^{32}$, T. Prakash ${ }^{117}$, S. Prince ${ }^{72}$, G. Prior ${ }^{113,114}$, D. Pugnere ${ }^{89}$, K. Qi ${ }^{168}$, X. Qian ${ }^{17}$, J. L. Raaf $^{61}$, R. Raboanary ${ }^{2}$, V. Radeka ${ }^{17}$, J. Rademacker ${ }^{16}$, B. Radics ${ }^{53}$, A. Rafique ${ }^{4}$, E. Raguzin ${ }^{17}$, M. Rai ${ }^{183}$, 
M. Rajaoalisoa ${ }^{37}$, I. Rakhno ${ }^{61}$, H. T. Rakotondramanana ${ }^{2}$, L. Rakotondravohitra ${ }^{2}$, Y. A. Ramachers ${ }^{183}$,

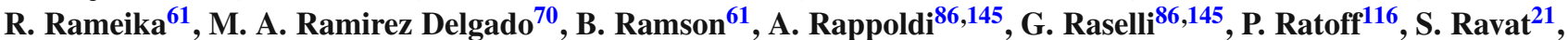
H. Razafinime ${ }^{2}$, J. S. Real ${ }^{69}$, B. Rebel ${ }^{186,61}$, D. Redondo ${ }^{22}$, M. Reggiani-Guzzo ${ }^{32}$, T. Rehak ${ }^{49}$, J. Reichenbacher ${ }^{164}$, S. D. Reitzner ${ }^{61}$, A. Renshaw ${ }^{74}$, S. Rescia ${ }^{17}$, F. Resnati ${ }^{21}$, A. Reynolds ${ }^{141}$, G. Riccobene ${ }^{87}$, L. C. J. Rice ${ }^{150}$, K. Rielage ${ }^{119}$, Y. Rigaut $^{53}$, D. Rivera ${ }^{146}$, L. Rochester ${ }^{159}$, M. Roda ${ }^{118}$, P. Rodrigues ${ }^{141}$, M. J. Rodriguez Alonso ${ }^{21}$, J. Rodriguez Rondon ${ }^{164}$, A. J. Roeth ${ }^{50}$, H. Rogers ${ }^{41}$, S. Rosauro-Alcaraz ${ }^{122}$, M. Rossella ${ }^{86,145}$, J. Rout ${ }^{103}$, S. Roy ${ }^{71}$, A. Rubbia ${ }^{53}$, C. Rubbia ${ }^{66}$, B. Russell ${ }^{117}$, J. Russell ${ }^{159}$, D. Ruterbories ${ }^{155}$, R. Saakyan ${ }^{178}$, S. Sacerdoti ${ }^{144}$, T. Safford ${ }^{126}$, N. Sahu ${ }^{96}$, P. Sala ${ }^{21,83}$, N. Samios ${ }^{17}$, M. C. Sanchez ${ }^{100}$, D. A. Sanders ${ }^{131}$, D. Sankey ${ }^{158}$, S. Santana ${ }^{152}$, M. Santos-Maldonado ${ }^{152}$, N. Saoulidou ${ }^{7}$, P. Sapienza ${ }^{87}$, C. Sarasty ${ }^{37}$, I. Sarcevic ${ }^{5}$, G. Savage $^{61}$, V. Savinov ${ }^{150}$, A. Scaramelli ${ }^{86}$, A. Scarff ${ }^{163}$, A. Scarpelli ${ }^{17}$, T. Schaffer ${ }^{129}$, H. Schellman ${ }^{61,140}$, P. Schlabach ${ }^{61}$, D. Schmitz ${ }^{35}$, K. Scholberg ${ }^{50}$, A. Schukraft ${ }^{61}$, E. Segreto ${ }^{32}$, J. Sensenig ${ }^{146}$, I. Seong ${ }^{26}$, A. Sergi ${ }^{13}$, F. Sergiampietri ${ }^{168}$, D. Sgalaberna ${ }^{53}$, M. H. Shaevitz ${ }^{42}$, S. Shafaq ${ }^{103}$, M. Shamma ${ }^{28}$, H. R. Sharma ${ }^{102}$, R. Sharma ${ }^{17}$, T. Shaw ${ }^{61}$, C. Shepherd-Themistocleous ${ }^{158}$, S. Shin ${ }^{104}$, D. Shooltz ${ }^{126}$, R. Shrock ${ }^{168}$, L. Simard ${ }^{115}$, N. Simos ${ }^{17}$, J. Sinclair ${ }^{11}$, G. Sinev ${ }^{50}$, J. Singh ${ }^{121}$, J. Singh ${ }^{121}$, V. Singh ${ }^{23,9}$, R. Sipos $^{21}$, F. W. Sippach ${ }^{42}$, G. Sirri ${ }^{78}$, A. Sitraka ${ }^{164}$, K. Siyeon ${ }^{36}$, D. Smargianaki ${ }^{168}$, A. Smith ${ }^{50}$, A. Smith ${ }^{31}$, E. Smith ${ }^{97}$, P. Smith ${ }^{97}$, J. Smolik ${ }^{44}$, M. Smy ${ }^{26}$, P. Snopok ${ }^{93}$, M. Soares Nunes ${ }^{32}$, H. Sobel ${ }^{26}$, M. Soderberg ${ }^{170}$, C. J. Solano Salinas ${ }^{98}$, S. Söldner-Rembold ${ }^{123}$, N. Solomey ${ }^{184}$, V. Solovov ${ }^{113,114}$, W. E. Sondheim ${ }^{119}$, M. Sorel ${ }^{77}$, J. Soto-Oton ${ }^{22}$, A. Sousa ${ }^{37}$, K. Soustruznik ${ }^{34}$, F. Spagliardi ${ }^{141}$, M. Spanu ${ }^{17}$, J. Spitz ${ }^{125}$, N. J. C. Spooner ${ }^{163}$, K. Spurgeon ${ }^{170}$, R. Staley ${ }^{13}$, M. Stancari ${ }^{61}$, L. Stanco ${ }^{85}$, H. M. Steiner ${ }^{117}$, J. Stewart ${ }^{17}$, B. Stillwell ${ }^{35}$, J. Stock ${ }^{164}$, F. Stocker ${ }^{21}$, T. Stokes ${ }^{120}$, M. Strait ${ }^{130}$, T. Strauss ${ }^{61}$,

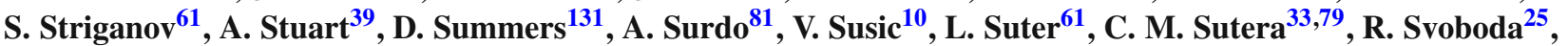
B. Szczerbinska ${ }^{172}$, A. M. Szelc ${ }^{123}$, R. Talaga ${ }^{4}$, H. A. Tanaka ${ }^{159}$, B. Tapia Oregui ${ }^{174}$, A. Tapper ${ }^{94}$, S. Tariq $^{61}$,

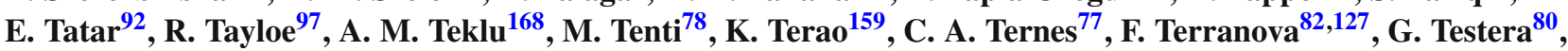
A. Thea ${ }^{158}$, J. L. Thompson ${ }^{163}$, C. Thorn ${ }^{17}$, S. C. Timm ${ }^{61}$, A. Tonazzo ${ }^{144}$, M. Torti ${ }^{82,127}$, M. Tortola ${ }^{77}$, F. Tortorici ${ }^{33,79}$, D. Totani ${ }^{61}$, M. Toups ${ }^{61}$, C. Touramanis ${ }^{118}$, J. Trevor ${ }^{30}$, W. H. Trzaska ${ }^{105}$, Y. T. Tsai ${ }^{159}$, Z. Tsamalaidze ${ }^{65}$, K. V. Tsang ${ }^{159}$, N. Tsverava ${ }^{65}$, S. Tufanli ${ }^{21}$, C. Tull ${ }^{117}$, E. Tyley ${ }^{163}$, M. Tzanov ${ }^{120}$, M. A. Uchida ${ }^{31}$, J. Urheim ${ }^{97}$, T. Usher ${ }^{159}$, M. R. Vagins ${ }^{110}$, P. Vahle ${ }^{185}$, G. A. Valdiviesso ${ }^{56}$, E. Valencia $^{185}$, Z. Vallari ${ }^{30}$, J. W. F. Valle ${ }^{77}$, S. Vallecorsa ${ }^{21}$, R. Van Berg ${ }^{146}$, R. G. Van de Water ${ }^{119}$, D. Vanegas Forero ${ }^{32}$, F. Varanini ${ }^{85}$,

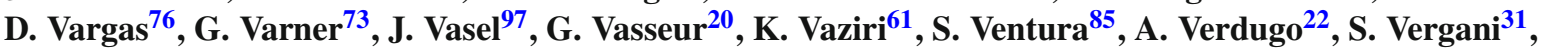
M. A. Vermeulen ${ }^{134}$, M. Verzocchi ${ }^{61}$, H. Vieira de Souza $^{32}$, C. Vignoli ${ }^{67}$, C. Vilela ${ }^{168}$, B. Viren ${ }^{17}$, T. Vrba ${ }^{44}$, T. Wachala ${ }^{133}$, A. V. Waldron ${ }^{94}$, M. Wallbank ${ }^{37}$, H. Wang ${ }^{27}$, J. Wang ${ }^{25}$, Y. Wang ${ }^{27}$, Y. Wang ${ }^{168}$, K. Warburton ${ }^{100}$, D. Warner ${ }^{41}$, M. Wascko ${ }^{94}$, D. Waters ${ }^{178}$, A. Watson ${ }^{13}$, P. Weatherly ${ }^{49}$, A. Weber ${ }^{141,158}$, M. Weber ${ }^{11}$, H. Wei ${ }^{17}$, A. Weinstein ${ }^{100}$, D. Wenman ${ }^{186}$, M. Wetstein ${ }^{100}$, M. R. While ${ }^{164}$, A. White ${ }^{173}$, L. H. Whitehead ${ }^{31}$, D. Whittington ${ }^{170}$, M. J. Wilking ${ }^{168}$, C. Wilkinson ${ }^{11, b}$, Z. Williams ${ }^{173}$, F. Wilson ${ }^{158}$, R. J. Wilson ${ }^{41}$, J. Wolcott ${ }^{176}$, T. Wongjirad ${ }^{176}$,

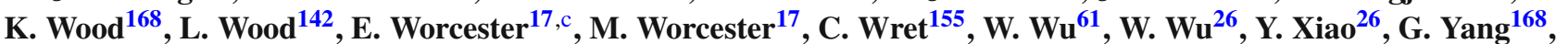
T. Yang ${ }^{61}$, N. Yershov ${ }^{88}$, K. Yonehara ${ }^{61}$, T. Young ${ }^{135}$, B. Yu ${ }^{17}$, J. Yu ${ }^{173}$, R. Zaki ${ }^{189}$, J. Zalesak ${ }^{43}$, L. Zambelli ${ }^{47}$, B. Zamorano ${ }^{68}$, A. Zani ${ }^{83}$, L. Zazueta ${ }^{185}$, G. P. Zeller ${ }^{61}$, J. Zennamo ${ }^{61}$, K. Zeug ${ }^{186}$, C. Zhang ${ }^{17}$, M. Zhao ${ }^{17}$, E. Zhivun ${ }^{17}$, G. Zhu ${ }^{139}$, E. D. Zimmerman ${ }^{40}$, M. Zito ${ }^{20}$, S. Zucchelli ${ }^{14,78}$, J. Zuklin ${ }^{43}$, V. Zutshi ${ }^{136}$, R. Zwaska ${ }^{61}$

\footnotetext{
${ }^{1}$ University of Amsterdam, 1098 XG Amsterdam, The Netherlands

2 University of Antananarivo, Antananarivo 101, Antananarivo, Madagascar

${ }^{3}$ Universidad Antonio Nariño, Bogotá, Colombia

${ }^{4}$ Argonne National Laboratory, Argonne, IL 60439, USA

${ }^{5}$ University of Arizona, Tucson, AZ 85721, USA

${ }^{6}$ Universidad Nacional de Asunción, San Lorenzo, Paraguay

${ }^{7}$ University of Athens, Zografou, GR 157 84, Greece

${ }^{8}$ Universidad del Atlántico, Atlántico, Colombia

${ }^{9}$ Banaras Hindu University, Varanasi 221 005, India

${ }^{10}$ University of Basel, 4056 Basel, Switzerland

${ }^{11}$ University of Bern, 3012 Bern, Switzerland

12 Beykent University, Istanbul, Turkey

${ }^{13}$ University of Birmingham, Birmingham B15 2TT, UK

${ }^{14}$ Università del Bologna, 40127 Bologna, Italy

15 Boston University, Boston, MA 02215, USA

${ }^{16}$ University of Bristol, Bristol BS8 1TL, UK

${ }^{17}$ Brookhaven National Laboratory, Upton, NY 11973, USA

${ }^{18}$ University of Bucharest, Bucharest, Romania

${ }^{19}$ Centro Brasileiro de Pesquisas Físicas, Rio de Janeiro, RJ 22290-180, Brazil
} 
${ }^{20}$ CEA/Saclay IRFU Institut de Recherche sur les Lois Fondamentales de l'Univers, 91191 Gif-sur-Yvette CEDEX, France

${ }^{21}$ CERN, The European Organization for Nuclear Research, 1211 Meyrin, Switzerland

${ }^{22}$ CIEMAT, Centro de Investigaciones Energéticas, Medioambientales y Tecnológicas, 28040 Madrid, Spain

${ }^{23}$ Central University of South Bihar, Gaya 824236, India

${ }^{24}$ University of California Berkeley, Berkeley, CA 94720, USA

${ }^{25}$ University of California Davis, Davis, CA 95616, USA

${ }^{26}$ University of California Irvine, Irvine, CA 92697, USA

${ }^{27}$ University of California Los Angeles, Los Angeles, CA 90095, USA

${ }^{28}$ University of California Riverside, Riverside, CA 92521, USA

${ }^{29}$ University of California Santa Barbara, Santa Barbara, California 93106, USA

${ }^{30}$ California Institute of Technology, Pasadena, CA 91125, USA

${ }^{31}$ University of Cambridge, Cambridge CB3 OHE, UK

32 Universidade Estadual de Campinas, Campinas, SP 13083-970, Brazil

${ }^{33}$ Università di Catania, 2 - 95131 Catania, Italy

${ }^{34}$ Institute of Particle and Nuclear Physics of the Faculty of Mathematics and Physics of the Charles University, 18000 Prague 8, Czech Republic

${ }^{35}$ University of Chicago, Chicago, IL 60637, USA

${ }^{36}$ Chung-Ang University, Seoul 06974, South Korea

37 University of Cincinnati, Cincinnati, OH 45221, USA

38 Centro de Investigación y de Estudios Avanzados del Instituto Politécnico Nacional (Cinvestav), Mexico City, Mexico

${ }^{39}$ Universidad de Colima, Colima, Mexico

40 University of Colorado Boulder, Boulder, CO 80309, USA

${ }^{41}$ Colorado State University, Fort Collins, CO 80523, USA

42 Columbia University, New York, NY 10027, USA

${ }^{43}$ Institute of Physics, Czech Academy of Sciences, 18200 Prague 8, Czech Republic

${ }^{44}$ Czech Technical University, 11519 Prague 1, Czech Republic

45 Dakota State University, Madison, SD 57042, USA

${ }^{46}$ University of Dallas, Irving, TX 75062-4736, USA

${ }^{47}$ Laboratoire d'Annecy-le-Vieux de Physique des Particules, CNRS/IN2P3 and Université Savoie Mont Blanc, 74941 Annecy-le-Vieux, France

48 Daresbury Laboratory, Cheshire WA4 4AD, UK

49 Drexel University, Philadelphia, PA 19104, USA

${ }^{50}$ Duke University, Durham, NC 27708, USA

${ }^{51}$ Durham University, Durham DH1 3LE, UK

52 Universidad EIA, Antioquia, Colombia

${ }^{53}$ ETH Zurich, Zurich, Switzerland

${ }^{54}$ University of Edinburgh, Edinburgh EH8 9YL, UK

55 Faculdade de Ciências da Universidade de Lisboa - FCUL, 1749-016 Lisboa, Portugal

56 Universidade Federal de Alfenas, Poços de Caldas, MG 37715-400, Brazil

${ }^{57}$ Universidade Federal de Goias, Goiania, GO 74690-900, Brazil

${ }^{58}$ Universidade Federal de São Carlos, Araras, SP 13604-900, Brazil

59 Universidade Federal do ABC, Santo André, SP 09210-580, Brazil

${ }^{60}$ Universidade Federal do Rio de Janeiro, Rio de Janeiro, RJ 21941-901, Brazil

${ }^{61}$ Fermi National Accelerator Laboratory, Batavia, IL 60510, USA

62 University of Florida, Gainesville, FL 32611-8440, USA

${ }^{63}$ Fluminense Federal University, 9 Icaraí, Niterói, RJ 24220-900, Brazil

${ }^{64}$ Università degli Studi di Genova, Genova, Italy

${ }^{65}$ Georgian Technical University, Tbilisi, Georgia

${ }^{66}$ Gran Sasso Science Institute, L'Aquila, Italy

${ }^{67}$ Laboratori Nazionali del Gran Sasso, L'Aquila, AQ, Italy

${ }^{68}$ University of Granada \& CAFPE, 18002 Granada, Spain

${ }^{69}$ University Grenoble Alpes, CNRS, Grenoble INP, LPSC-IN2P3, 38000 Grenoble, France

${ }^{70}$ Universidad de Guanajuato, Guanajuato C.P. 37000, Mexico

${ }^{71}$ Harish-Chandra Research Institute, Jhunsi, Allahabad 211 019, India

72 Harvard University, Cambridge, MA 02138, USA

${ }^{73}$ University of Hawaii, Honolulu, HI 96822, USA

${ }^{74}$ University of Houston, Houston, TX 77204, USA

${ }^{75}$ University of Hyderabad, Gachibowli, Hyderabad 500 046, India

${ }^{76}$ Institut de Fìsica d'Altes Energies, Barcelona, Spain

77 Instituto de Fisica Corpuscular, 46980, Paterna Valencia, Spain

${ }^{78}$ Istituto Nazionale di Fisica Nucleare Sezione di Bologna, 40127 Bologna, BO, Italy

${ }^{79}$ Istituto Nazionale di Fisica Nucleare Sezione di Catania, 95123 Catania, Italy

${ }^{80}$ Istituto Nazionale di Fisica Nucleare Sezione di Genova, 16146 Genova, GE, Italy

${ }^{81}$ Istituto Nazionale di Fisica Nucleare Sezione di Lecce, 73100 Lecce, Italy

82 Istituto Nazionale di Fisica Nucleare Sezione di Milano Bicocca, 3-20126 Milan, Italy

${ }^{83}$ Istituto Nazionale di Fisica Nucleare Sezione di Milano, 20133 Milan, Italy

${ }^{84}$ Istituto Nazionale di Fisica Nucleare Sezione di Napoli, 80126 Naples, Italy 
${ }^{85}$ Istituto Nazionale di Fisica Nucleare Sezione di Padova, 35131 Padua, Italy

${ }^{86}$ Istituto Nazionale di Fisica Nucleare Sezione di Pavia, 27100 Pavia, Italy

${ }^{87}$ Istituto Nazionale di Fisica Nucleare Laboratori Nazionali del Sud, 95123 Catania, Italy

${ }^{88}$ Institute for Nuclear Research of the Russian Academy of Sciences, Moscow 117312, Russia

${ }^{89}$ Institut de Physique des 2 Infinis de Lyon, 69622 Villeurbanne, France

${ }^{90}$ Institute for Research in Fundamental Sciences, Tehran, Iran

${ }^{91}$ Instituto Superior Técnico-IST, Universidade de Lisboa, Lisboa, Portugal

92 Idaho State University, Pocatello ID 83209, USA

${ }^{93}$ Illinois Institute of Technology, Chicago, IL 60616, USA

94 Imperial College of Science Technology and Medicine, London SW7 2BZ, UK

95 Indian Institute of Technology Guwahati, Guwahati 781 039, India

${ }^{96}$ Indian Institute of Technology Hyderabad, Hyderabad 502285, India

97 Indiana University, Bloomington, IN 47405, USA

${ }^{98}$ Universidad Nacional de Ingeniería, Lima 25, Peru

${ }^{99}$ University of Iowa, Iowa City, IA 52242, USA

${ }^{100}$ Iowa State University, Ames, IA 50011, USA

${ }^{101}$ Iwate University, Morioka, Iwate 020-8551, Japan

102 University of Jammu, Jammu 180006, India

103 Jawaharlal Nehru University, New Delhi 110067, India

104 Jeonbuk National University, Jeonrabuk-do 54896, South Korea

105 University of Jyvaskyla, 40014 Jyvaskyla, Finland

106 High Energy Accelerator Research Organization (KEK), Ibaraki 305-0801, Japan

107 Korea Institute of Science and Technology Information, Daejeon 34141, South Korea

${ }^{108}$ K L University, Vaddeswaram, Andhra Pradesh 522502, India

${ }^{109}$ Kansas State University, Manhattan, KS 66506, USA

${ }^{110}$ Kavli Institute for the Physics and Mathematics of the Universe, Kashiwa, Chiba 277-8583, Japan

111 National Institute of Technology, Kure College, Hiroshima 737-8506, Japan

112 Kyiv National University, 01601 Kyiv, Ukraine

113 Laboratório de Instrumentação e Física Experimental de Partículas, 1649-003 Lisboa, Portugal

114 Laboratório de Instrumentação e Física Experimental de Partículas, 3004-516 Coimbra, Portugal

115 Laboratoire de l'Accélérateur Linéaire, 91440 Orsay, France

116 Lancaster University, Lancaster LA1 4YB, UK

117 Lawrence Berkeley National Laboratory, Berkeley, CA 94720, USA

118 University of Liverpool, L69 7ZE Liverpool, UK

${ }^{119}$ Los Alamos National Laboratory, Los Alamos, NM 87545, USA

${ }^{120}$ Louisiana State University, Baton Rouge, LA 70803, USA

${ }^{121}$ University of Lucknow, Uttar Pradesh 226007, India

122 Madrid Autonoma University and IFT UAM/CSIC, 28049 Madrid, Spain

${ }^{123}$ University of Manchester, Manchester M13 9PL, UK

124 Massachusetts Institute of Technology, Cambridge, MA 02139, USA

125 University of Michigan, Ann Arbor, MI 48109, USA

${ }^{126}$ Michigan State University, East Lansing, MI 48824, USA

${ }^{127}$ Università del Milano-Bicocca, 20126 Milan, Italy

${ }^{128}$ Università degli Studi di Milano, 20133 Milan, Italy

129 University of Minnesota Duluth, Duluth, MN 55812, USA

${ }^{130}$ University of Minnesota Twin Cities, Minneapolis, MN 55455, USA

131 University of Mississippi, University, MS 38677, USA

132 University of New Mexico, Albuquerque, NM 87131, USA

133 H. Niewodniczański Institute of Nuclear Physics, Polish Academy of Sciences, Cracow, Poland

134 Nikhef National Institute of Subatomic Physics, 1098 XG Amsterdam, Netherlands

135 University of North Dakota, Grand Forks, ND 58202-8357, USA

${ }^{136}$ Northern Illinois University, DeKalb, Illinois 60115, USA

137 Northwestern University, Evanston, Il 60208, USA

${ }^{138}$ University of Notre Dame, Notre Dame, IN 46556, USA

139 Ohio State University, Columbus, OH 43210, USA

140 Oregon State University, Corvallis, OR 97331, USA

${ }^{141}$ University of Oxford, Oxford OX1 3RH, UK

142 Pacific Northwest National Laboratory, Richland, WA 99352, USA

143 Universtà degli Studi di Padova, 35131 Padua, Italy

144 Université de Paris, CNRS, Astroparticule et Cosmologie, 75006 Paris, France

145 Università degli Studi di Pavia, 27100 Pavia, PV, Italy

146 University of Pennsylvania, Philadelphia, PA 19104, USA

147 Pennsylvania State University, University Park, PA 16802, USA

148 Physical Research Laboratory, Ahmedabad 380 009, India

${ }^{149}$ Università di Pisa, 56127 Pisa, Italy

${ }^{150}$ University of Pittsburgh, Pittsburgh, PA 15260, USA 
${ }^{151}$ Pontificia Universidad Católica del Perú, Lima, Peru

152 University of Puerto Rico, Mayaguez 00681, Puerto Rico, USA

153 Punjab Agricultural University, Ludhiana 141004, India

154 Radboud University, NL-6525, AJ Nijmegen, Netherlands

155 University of Rochester, Rochester, NY 14627, USA

156 Royal Holloway College, London TW20 0EX, UK

157 Rutgers University, Piscataway, NJ 08854, USA

158 STFC Rutherford Appleton Laboratory, Didcot OX11 0QX, UK

${ }^{159}$ SLAC National Accelerator Laboratory, Menlo Park, CA 94025, USA

${ }^{160}$ Sanford Underground Research Facility, Lead, SD 57754, USA

${ }^{161}$ Università del Salento, 73100 Lecce, Italy

162 Universidad Sergio Arboleda, 11022 Bogotá, Colombia

163 University of Sheffield, Sheffield S3 7RH, UK

164 South Dakota School of Mines and Technology, Rapid City, SD 57701, USA

165 South Dakota State University, Brookings, SD 57007, USA

166 University of South Carolina, Columbia, SC 29208, USA

${ }^{167}$ Southern Methodist University, Dallas, TX 75275, USA

168 Stony Brook University, SUNY, Stony Brook, New York 11794, USA

${ }^{169}$ University of Sussex, Brighton BN1 9RH, UK

170 Syracuse University, Syracuse, NY 13244, USA

${ }^{171}$ University of Tennessee, Knoxville, TN 37996, USA

172 Texas A\&M University - Corpus Christi, Corpus Christi, TX 78412, USA

173 University of Texas at Arlington, Arlington, TX 76019, USA

${ }^{174}$ University of Texas at Austin, Austin, TX 78712, USA

175 University of Toronto, Toronto, Ontario M5S 1A1, Canada

176 Tufts University, Medford, MA 02155, USA

${ }^{177}$ Universidade Federal de São Paulo, 09913-030 São Paulo, Brazil

178 University College London, London WC1E 6BT, UK

${ }^{179}$ Valley City State University, Valley City, ND 58072, USA

${ }^{180}$ Variable Energy Cyclotron Centre, 700064 West Bengal, India

181 Virginia Tech, Blacksburg, VA 24060, USA

182 University of Warsaw, 00-927 Warsaw, Poland

183 University of Warwick, Coventry CV4 7AL, UK

184 Wichita State University, Wichita, KS 67260, USA

185 William and Mary, Williamsburg, VA 23187, USA

${ }^{186}$ University of Wisconsin Madison, Madison, WI 53706, USA

187 Yale University, New Haven, CT 06520, USA

188 Yerevan Institute for Theoretical Physics and Modeling, Yerevan 0036, Armenia

189 York University, Toronto M3J 1P3, Canada

Received: 2 June 2020 / Accepted: 10 September 2020 / Published online: 22 October 2020

(C) The Author(s) 2020

\begin{abstract}
The sensitivity of the Deep Underground Neutrino Experiment (DUNE) to neutrino oscillation is determined, based on a full simulation, reconstruction, and event selection of the far detector and a full simulation and parameterized analysis of the near detector. Detailed uncertainties due to the flux prediction, neutrino interaction model, and detector effects are included. DUNE will resolve the neutrino mass ordering to a precision of $5 \sigma$, for all $\delta_{\mathrm{CP}}$ values, after 2 years of running with the nominal detector design and beam configuration. It has the potential to observe charge-parity violation in the neutrino sector to a precision of $3 \sigma(5 \sigma)$ after an exposure of 5 (10) years,

\footnotetext{
a e-mail: marshall@1bl.gov

b e-mail: callum.wilkinson@lhep.unibe.ch (corresponding author)

c e-mail: etw@bnl.gov
}

for $50 \%$ of all $\delta_{\mathrm{CP}}$ values. It will also make precise measurements of other parameters governing long-baseline neutrino oscillation, and after an exposure of 15 years will achieve a similar sensitivity to $\sin ^{2} 2 \theta_{13}$ to current reactor experiments.

\section{Introduction}

The Deep Underground Neutrino Experiment (DUNE) is a next-generation, long-baseline neutrino oscillation experiment which will carry out a detailed study of neutrino mixing utilizing high-intensity $v_{\mu}$ and $\bar{v}_{\mu}$ beams measured over a long baseline. DUNE is designed to make significant contributions to the completion of the standard threeflavor picture by measuring all the parameters govern- 
ing $v_{1}-v_{3}$ and $v_{2}-v_{3}$ mixing in a single experiment. Its main scientific goals are the definitive determination of the neutrino mass ordering, the definitive observation of charge-parity symmetry violation (CPV) for more than 50\% of possible true values of the charge-parity violating phase, $\delta_{\mathrm{CP}}$, and precise measurement of oscillation parameters, particularly $\delta_{\mathrm{CP}}, \sin ^{2} 2 \theta_{13}$, and the octant of $\theta_{23}$. These measurements will help guide theory in understanding if there are new symmetries in the neutrino sector and whether there is a relationship between the generational structure of quarks and leptons [1]. Observation of CPV in neutrinos would be an important step in understanding the origin of the baryon asymmetry of the universe [2,3].

The DUNE experiment will observe neutrinos from a high-power neutrino beam peaked at $\sim 2.5 \mathrm{GeV}$ but with a broad range of neutrino energies, a near detector (ND) located at Fermi National Accelerator Laboratory, in Batavia, Illinois, USA, and a large liquid argon time-projection chamber (LArTPC) far detector (FD) located at the $4850 \mathrm{ft}$ level of Sanford Underground Research Facility (SURF), in Lead, South Dakota, USA, $1285 \mathrm{~km}$ from the neutrino production point. The neutrino beam provided by Long-Baseline Neutrino Facility (LBNF) [4] is produced using protons from Fermilab's Main Injector, which are guided onto a graphite target, and a traditional horn-focusing system to select and focus particles produced in the target [5]. The polarity of the focusing magnets can be reversed to produce a beam dominated by either muon neutrinos or muon antineutrinos. A highly capable ND will constrain many systematic uncertainties for the oscillation analysis. The 40-kt (fiducial) FD is composed of four $10 \mathrm{kt}$ (fiducial) LArTPC modules [6-8]. The deep underground location of the FD reduces cosmogenic and atmospheric sources of background, which also provides sensitivity to nucleon decay and low-energy neutrino detection, for example, the possible observation of neutrinos from a core-collapse supernova [5].

The entire complement of neutrino oscillation experiments to date has measured five of the neutrino mixing parameters [9-11]: the three mixing angles $\theta_{12}, \theta_{23}$, and $\theta_{13}$, and the two squared-mass differences $\Delta m_{21}^{2}$ and $\left|\Delta m_{31}^{2}\right|$, where $\Delta m_{i j}^{2}=m_{i}^{2}-m_{j}^{2}$ is the difference between the squares of the neutrino mass states in $\mathrm{eV}^{2}$. The neutrino mass ordering (i.e., the sign of $\Delta m_{31}^{2}$ ) is unknown, though recent results show a weak preference for the normal ordering [12-14]. The value of $\delta_{\mathrm{CP}}$ is not well known, though neutrino oscillation data are beginning to provide some information on its value $[12,15]$.

The oscillation probability of $v_{\mu} \rightarrow v_{e}$ through matter in the standard three-flavor model and a constant density approximation is, to first order [16]:

$$
\begin{aligned}
P\left(\bar{v}_{\mu} \rightarrow\left(\bar{v}_{e}\right) \simeq\right. & \sin ^{2} \theta_{23} \sin ^{2} 2 \theta_{13} \\
& \frac{\sin ^{2}\left(\Delta_{31}-a L\right)}{\left(\Delta_{31}-a L\right)^{2}} \Delta_{31}^{2} \\
& +\sin 2 \theta_{23} \sin 2 \theta_{13} \sin 2 \theta_{12} \\
& \times \frac{\sin \left(\Delta_{31}-a L\right)}{\left(\Delta_{31}-a L\right)} \Delta_{31} \\
& \times \frac{\sin (a L)}{(a L)} \Delta_{21} \cos \left(\Delta_{31} \pm \delta_{\mathrm{CP}}\right) \\
& +\cos ^{2} \theta_{23} \sin ^{2} 2 \theta_{12} \frac{\sin ^{2}(a L)}{(a L)^{2}} \Delta_{21}^{2},
\end{aligned}
$$

where

$a= \pm \frac{G_{\mathrm{F}} N_{e}}{\sqrt{2}} \approx \pm \frac{1}{3500 \mathrm{~km}}\left(\frac{\rho}{3.0 \mathrm{~g} / \mathrm{cm}^{3}}\right)$,

$G_{\mathrm{F}}$ is the Fermi constant, $N_{e}$ is the number density of electrons in the Earth's crust, $\Delta_{i j}=1.267 \Delta m_{i j}^{2} L / E_{v}, L$ is the baseline in $\mathrm{km}$, and $E_{v}$ is the neutrino energy in $\mathrm{GeV}$. Both $\delta_{\mathrm{CP}}$ and $a$ terms are positive for $v_{\mu} \rightarrow v_{e}$ and negative for $\bar{v}_{\mu} \rightarrow \bar{v}_{e}$ oscillations; i.e., a neutrino-antineutrino asymmetry is introduced both by CPV $\left(\delta_{\mathrm{CP}}\right)$ and the matter effect (a). The origin of the matter effect asymmetry is simply the presence of electrons and absence of positrons in the Earth $[17,18]$. The (anti-)electron neutrino appearance probability is shown in Fig. 1 at the DUNE baseline of $1285 \mathrm{~km}$ as a function of neutrino energy for several values of $\delta_{\mathrm{CP}}$.

DUNE has a number of features that give it unique physics reach, complementary to other existing and planned experiments [19-21]. Its broad-band beam makes it sensitive to the shape of the oscillation spectrum for a range of neutrino energies. DUNE's relatively high energy neutrino beam enhances the size of the matter effect and will allow DUNE to measure $\delta_{\mathrm{CP}}$ and the mass ordering simultaneously. The unique LArTPC detector technology will enhance the resolution on DUNE's measurement of the value of $\delta_{\mathrm{CP}}$, and along with the increased neutrino energy, gives DUNE a different set of systematic uncertainties to other experiments, making DUNE complementary with them.

This paper describes studies that quantify DUNE's expected sensitivity to long-baseline neutrino oscillation, using the accelerator neutrino beam. Note that atmospheric neutrino samples would provide additional sensitivity to some of the same physics, but are not included in this work. The flux simulation and associated uncertainties are described in Sect. 2. Section 3 describes the neutrino interaction model and systematic variations. The near and far detector simulation, reconstruction, and event selections are described in Sects. 4 and 5, respectively, with a nominal set of event rate predictions given in Sect. 6. Detector uncertainties are described in Sect. 7. The methods used to extract oscillation sensitivities are described in Sect. 8. The primary 

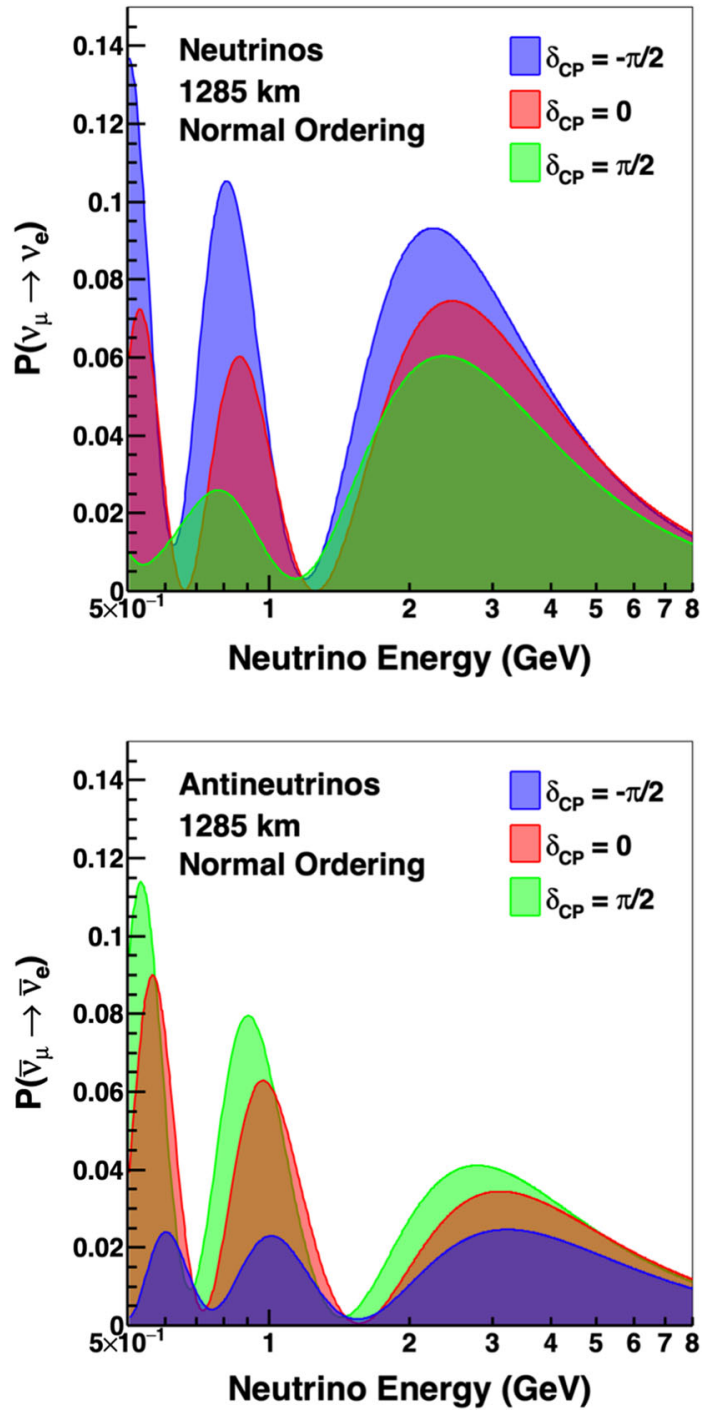

Fig. 1 The appearance probability at a baseline of $1285 \mathrm{~km}$, as a function of neutrino energy, for $\delta_{\mathrm{CP}}=-\pi / 2$ (blue), 0 (red), and $\pi / 2$ (green), for neutrinos (top) and antineutrinos (bottom), for normal ordering

sensitivity results are presented in Sect. 9. We present our conclusions in Sect. 10.

\section{Neutrino beam flux and uncertainties}

The expected neutrino flux is generated using G4LBNF [5, 22], a Geant4-based [23] simulation of the LBNF neutrino beam. The simulation uses a detailed description of the LBNF optimized beam design [5], which includes a target and horns designed to maximize sensitivity to CPV given the physical constraints on the beamline design.

Neutrino fluxes for neutrino-enhanced, forward horn current (FHC), and antineutrino-enhanced, reverse horn current (RHC), configurations of LBNF are shown in Fig. 2. Uncertainties on the neutrino fluxes arise primarily from uncertain-
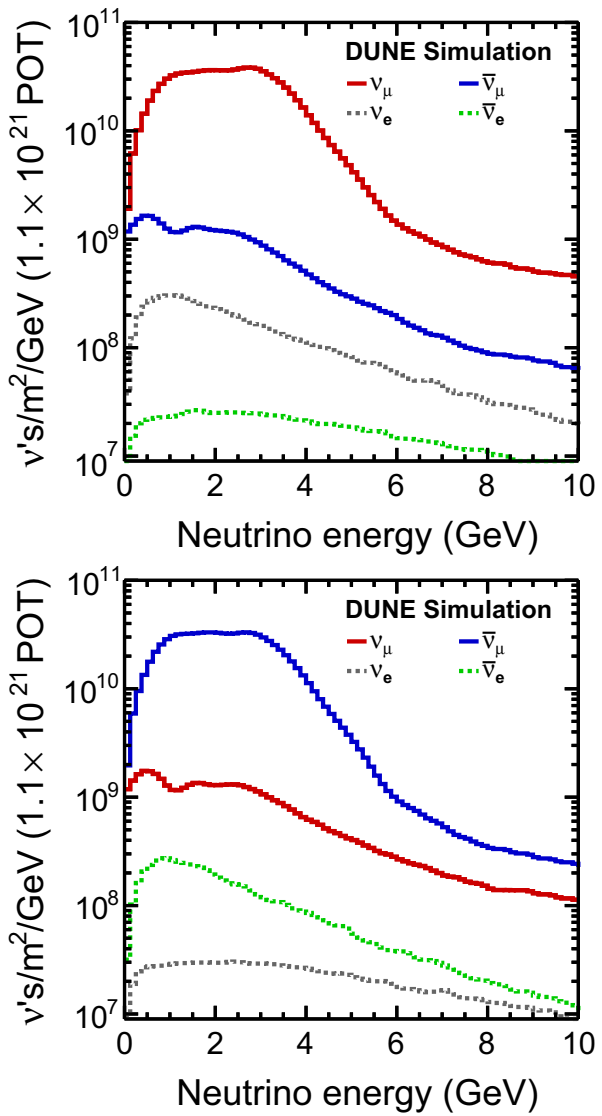

Fig. 2 Neutrino fluxes at the FD for neutrino-enhanced, FHC, beam running (top) and antineutrino, RHC, beam running (bottom)

ties in hadrons produced off the target and uncertainties in the design parameters of the beamline, such as horn currents and horn and target positioning (commonly called "focusing uncertainties") [5]. Given current measurements of hadron production and LBNF estimates of alignment tolerances, flux uncertainties are approximately $8 \%$ at the first oscillation maximum and $12 \%$ at the second. These uncertainties are highly correlated across energy bins and neutrino flavors. The unoscillated fluxes at the ND and FD are similar, but not identical. The relationship is well understood, and flux uncertainties mostly cancel for the ratio of fluxes between the two detectors. Uncertainties on the ratio are dominated by focusing uncertainties and are $\sim 1 \%$ or smaller except at the falling edge of the focusing peak $(\sim 4 \mathrm{GeV})$, where they rise to $2 \%$. The rise is due to the presence of many particles which are not strongly focused by the horns in this energy region, which are particularly sensitive to focusing and alignment uncertainties. The near-to-far flux ratio and uncertainties on this ratio are shown in Fig. 3.

Beam-focusing and hadron-production uncertainties on the flux prediction are evaluated by reproducing the full beamline simulation many times with variations of the input model according to those uncertainties. The resultant uncer- 

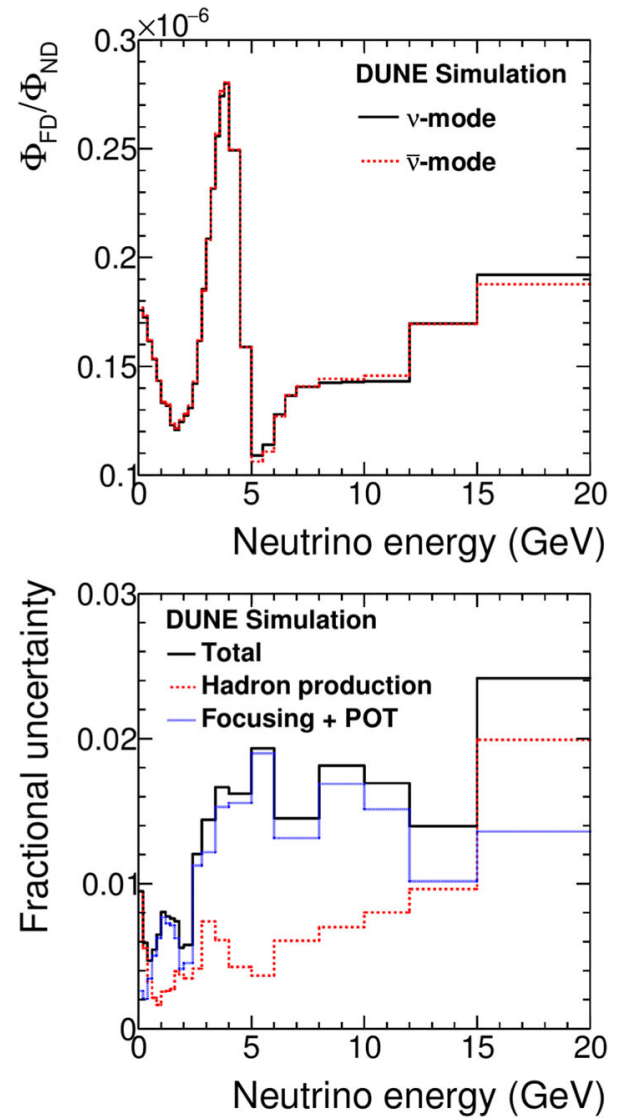

Fig. 3 Ratio of ND and FD fluxes show for the muon neutrino component of the FHC flux and the muon antineutrino component of the RHC flux (top) and uncertainties on the FHC muon neutrino ratio (bottom)

tainty on the neutrino flux prediction is described through a covariance matrix, where each bin corresponds to an energy range of a particular beam mode and neutrino species, separated by flux at the ND and FD. The output covariance matrix has $208 \times 208$ bins, despite having only $\sim 30$ input uncertainties. To reduce the number of parameters used in the fit, the covariance matrix is diagonalized, and each principal component is treated as an uncorrelated nuisance parameter. The 208 principal components are ordered by the magnitude of their corresponding eigenvalues, which is the variance along the principal component (eigenvector) direction, and only the first $\sim 30$ are large enough that they need to be included. This was validated by including more flux parameters and checking that there was no significant change to the sensitivity for a small number of test cases. By the 10th principal component, the eigenvalue is $1 \%$ of the largest eigenvalue. As may be expected, the largest uncertainties correspond to the largest principal components as shown in Fig. 4. The largest principal component (component 0 ) matches the hadron production uncertainty on nucleon-nucleus interactions in a phase space region not covered by data. Components 3 and 7 correspond to the data-constrained uncertainty on proton interactions in the target producing pions and kaons, respectively. Compo-

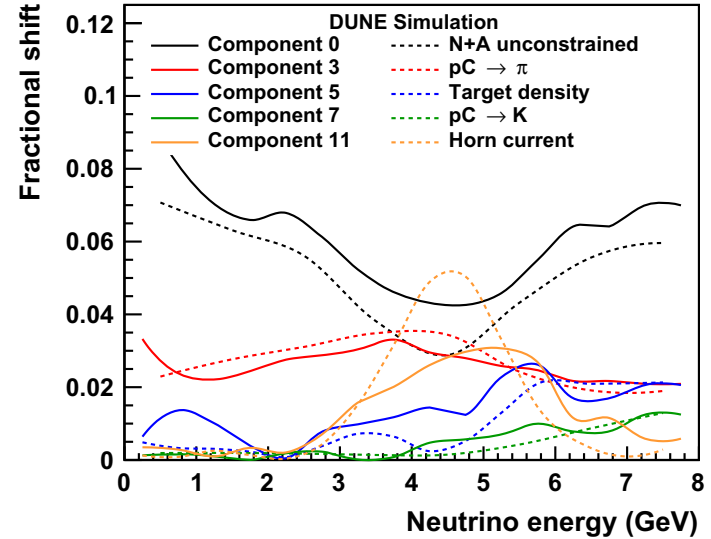

Fig. 4 Select flux principal components are compared to specific underlying uncertainties from the hadron production and beam focusing models. Note that while these are shown as positive shifts, the absolute sign is arbitrary

nents 5 and 11 correspond to two of the largest focusing uncertainties, the density of the target and the horn current, respectively. Other components not shown either do not fit a single uncertain parameter or may represent two or more degenerate systematics or ones that produce anti-correlations in neighboring energy bins.

Future hadron production measurements are expected to improve the quality of, and the resulting constraints on, these flux uncertainty estimates. Approximately $40 \%$ of the interactions that produce neutrinos in the LBNF beam simulation have no direct data constraints. Large uncertainties are assumed for these interactions. The largest unconstrained sources of uncertainty are proton quasielastic interactions and pion and kaon rescattering in beamline materials. The proposed EMPHATIC experiment [24] at Fermilab will be able to constrain quasielastic and low-energy interactions that dominate the lowest neutrino energy bins. The NA61 experiment at CERN has taken data that will constrain many higher energy interactions, and also plans to measure hadrons produced on a replica LBNF target, which would provide tight constraints on all interactions occurring in the target. A similar program at NA61 has reduced flux uncertainties for the T2K experiment from $\sim 10$ to $\sim 5 \%$ [25]. Another proposed experiment, the LBNF spectrometer [26], would measure hadrons after both production and focusing in the horns to further constrain the hadron production uncertainties, and could also be used to experimentally assess the impact of shifted alignment parameters on the focused hadrons (rather than relying solely on simulation).

\section{Neutrino interaction model and uncertainties}

A framework for considering the impact of neutrino interaction model uncertainties on the oscillation analysis has 
been developed. The default interaction model is implemented in v2.12.10 of the GENIE generator [27,28]. Variations in the cross sections are implemented in various ways: using GENIE reweighting parameters (sometimes referred to as "GENIE knobs"); with ad hoc weights of events that are designed to parameterize uncertainties or cross-section corrections currently not implemented within GENIE; or through discrete alternative model comparisons. The latter are achieved through alternative generators, alternative GENIE configurations, or custom weightings, which made extensive use of the NUISANCE package [29] in their development.

The interaction model components and uncertainties can be divided into seven groups: (1) initial state, (2) hard scattering and nuclear modifications to the quasielastic, or one-particle one-hole ( $1 p 1 h)$ process, (3) multinucleon, or two-particle two-hole ( $2 p 2 h)$, hard scattering processes, (4) hard scattering in pion production processes, (5) higher invariant mass $(W)$ and neutral current (NC) processes, (6) final-state interactions (FSI), (7) neutrino flavor dependent differences. Uncertainties are intended to reflect current theoretical freedom, deficiencies in implementation, and/or current experimental knowledge.

The default nuclear model in GENIE describing the initial state of nucleons in the nucleus is the Bodek-Ritchie global Fermi gas model [30]. There are significant deficiencies that are known in global Fermi gas models: these include a lack of consistent incorporation of the high-momentum tails in the nucleon momentum distribution that result from correlations among nucleons; the lack of correlation between location within the nucleus and momentum of the nucleon; and an incorrect relationship between momentum and energy of the off-shell, bound nucleon within the nucleus. They have also been shown to agree poorly with neutrino-nucleus scattering data [31]. GENIE modifies the nucleon momentum distribution empirically to account for short-range correlation effects, which populates the high-momentum tail above the Fermi cutoff, but the other deficiencies persist. Alternative initial state models, such as spectral functions [32,33], the mean field model of GiBUU [34], or continuum random phase approximation (CRPA) calculations [35] may provide better descriptions of the nuclear initial state [36], but are not considered further here.

The primary uncertainties considered in $1 p 1 h$ interactions $\left(v_{l}+n \rightarrow l^{-}+p, \bar{v}_{l}+p \rightarrow l^{+}+n\right)$ are the axial form factor of the nucleon and the nuclear screening-from the socalled random phase approximation (RPA) calculations-of low momentum transfer reactions. The Valencia group's [37,38] description of RPA comes from summation of $W^{ \pm}$self-energy terms. In practice, this modifies the $1 p 1 h$ (quasielastic) cross section in a non-trivial way, with associated uncertainties presented in Ref. [39], which were evaluated as a function of $Q^{2}$. Here we use T2K's 2017/8 param- eterization of the Valencia RPA effect [12]. The shape of the correction and error is parameterized with a third-order Bernstein polynomial up to $Q^{2}=1.2 \mathrm{GeV}^{2}$ where the form transitions to a decaying exponential. The BeRPA (Bernstein RPA) function has three parameters controlling the behavior at increasing $Q^{2}$ (A, B and D), a fourth parameter (E) that controls the high- $Q^{2}$ tail, and a fifth (U), which changes the position at which the behaviour changes from polynomial to exponential. The BeRPA parameterization modifies the central value of the model prediction, as decribed in Table 3 . BeRPA parameters $E$ and $U$ are not varied in the analysis described here, the parameters $\mathrm{A}$ and $\mathrm{B}$ have a prefit uncertainty of $20 \%$, and D has a prefit unertainty of $15 \%$. The axial form factor parameterization we use, a dipole, is known to be inadequate [40]. However, the convolution of BeRPA uncertainties with the limited axial form factor uncertainties do provide more freedom as a function of $Q^{2}$, and the two effects combined likely provide adequate freedom for the $Q^{2}$ shape in quasielastic events. BBBA05 vector form factors are used [41].

The $2 p 2 h$ contribution to the cross section comes from the Valencia model [37,38], the implementation in GENIE is described in Ref. [42]. However, MINERvA [43] and NOvA [44] have shown that this model underpredicts observed event rates on carbon. The extra strength from the "MINERvA tune" to $2 p 2 h$ is applied as a two-dimensional Gaussian in $\left(q_{0}, q_{3}\right)$ space, where $q_{0}$ is the energy transfer from the leptonic system, and $q_{3}$ is the magnitude of the three momentum transfer) to fit reconstructed MINERvA CC-inclusive data [43]. Reasonable predictions of MINERvA's data are found by attributing the missing strength to any of $2 p 2 h$ from $n p$ initial state pairs, $2 p 2 h$ from $n n$ initial state pairs, or $1 p 1 h$ (quasielastic) processes. The default tune uses an enhancement of the $n p$ and $n n$ initial strengths in the ratio predicted by the Valencia model, and alternative systematic variation tunes ("MnvTune" 1-3) attribute the missing strength to the individual interaction processes above. We add uncertainties for the energy dependence of this missing strength based on the MINERvA results [43], and assume a generic form for the energy dependence of the cross section using the "A" and "B" terms taken from Ref. [45]. These uncertainties are labeled $E_{2 p 2 h}$ and are separated for neutrinos and antineutrinos. We add uncertainties on scaling the $2 p 2 h$ prediction from carbon to argon on electron-scattering measurements of short-range correlated (SRC) pairs taken on multiple targets [46], separately for neutrinos $(\operatorname{ArC} 2 p 2 h v)$ and antineutrinos $(\operatorname{ArC} 2 p 2 h \bar{v})$.

GENIE uses a modified version of the Rein-Sehgal (R-S) model for pion production [47], including only the 16 resonances recommended by the Particle Data group [48], and excluding interferences between resonances. The cross section is cut off at invariant masses, $W \geq 1.7 \mathrm{GeV}(2 \mathrm{GeV}$ in the original R-S model). No in-medium modifications to the 
resonances are included, and by default they decay isotropically in their rest frame, although there is a parameter denoted here as " $\theta_{\pi}$ from $\Delta$-decay", for changing the angular distribution of pions produced through $\Delta$ resonance decays to match the experimentally observed distributions used in the original R-S paper [47]. Resonance decays to $\eta$ and $\gamma$ (plus a nucleon) are included from Ref. [48]. We use a tuning of the GENIE model to reanalyzed neutrino-deuterium bubble chamber data $[49,50]$ as our base model, as noted in Table 3 . We note that an improved Rein-Sehgal-like resonance model has been developed [51], and has been implemented in Monte Carlo generators, although is not used as the default model in the present work.

The deep inelastic scattering (DIS) model implemented in GENIE uses the Bodek-Yang parametrization [52], using GRV98 parton distribution functions [53]. Hadronization is described by the AKGY model [54], which uses the KNO scaling model [55] for invariant masses $W \leq 2.3 \mathrm{GeV}$ and PYTHIA6 [56] for invariant masses $W \geq 3 \mathrm{GeV}$, with a smooth transition between the two for intermediate invariant masses. A number of variable parameters affecting DIS processes are included in GENIE, as listed in Table 3, and described in Ref. [52]. In GENIE, the DIS model is extrapolated to all values of invariant mass, and replaces the nonresonant background to pion production in the R-S model.

The NOvA experiment [57] developed uncertainties beyond those provided by GENIE to describe their single pion to DIS transition region data. We follow their findings, and implement separate, uncorrelated uncertainties for all perturbations of 1,2 , and $\geq 3$ pion final states, $\mathrm{CC} / \mathrm{NC}$, neutrinos/antineutrinos, and interactions on protons/neutrons, with the exception of $\mathrm{CC}$ neutrino 1-pion production, where interactions on protons and neutrons are merged, following [50], which modifies the central value of the model prediction, as listed in Table 3 . This leads to 23 distinct uncertainty channels with a label to denote the process it affects: $\operatorname{NR}[v, \bar{v}]$ $[C C, N C][n, p][1 \pi, 2 \pi, 3 \pi]$. Each channel has an uncertainty of $50 \%$ for $W \leq 3 \mathrm{GeV}$, and an uncertainty which drops linearly above $W=3 \mathrm{GeV}$ until it reaches a flat value of $5 \%$ at $W=5 \mathrm{GeV}$, where external measurements better constrain this process.

GENIE includes a large number of final state uncertainties on its final state cascade model [58-60], which are summarized in Table 2. A recent comparison of the underlying interaction probabilities used by GENIE is compared with other available simulation packages in Ref. [61].

The cross sections include terms proportional to the lepton mass, which are significant contributors at low energies where quasielastic processes dominate. Some of the form factors in these terms have significant uncertainties in the nuclear environment. Ref. [62] ascribes the largest possible effect to the presence of poorly constrained secondclass current vector form factors in the nuclear environ- ment, and proposes a variation in the cross section ratio of $\sigma_{\mu} / \sigma_{e}$ of $\pm 0.01 / \operatorname{Max}\left(0.2 \mathrm{GeV}, E_{\nu}\right)$ for neutrinos and $\mp 0.018 / \operatorname{Max}\left(0.2 \mathrm{GeV}, E_{v}\right)$ for antineutrinos. Note the anticorrelation of the effect in neutrinos and antineutrinos. This parameter is labeled $v_{e} / \bar{v}_{e}$ norm.

An additional normalization uncertainty ( $\mathrm{NC}$ norm.) of $20 \%$ is applied to all NC events at the ND in this analysis to investigate whether the small contamination of $\mathrm{NC}$ events which passed the simple selection cuts had an effect on the analysis. Although a similar systematic could have been included (uncorrelated) at the FD, it was not in this analysis.

Finally, some electron-neutrino interactions occur at fourmomentum transfers where a corresponding muon-neutrino interaction is kinematically forbidden, therefore the nuclear response has not been constrained by muon-neutrino crosssection measurements. This region at lower neutrino energies has a significant overlap with the Bodek-Ritchie tail of the nucleon momentum distribution in the Fermi gas model [30]. There are significant uncertainties in this region, both from the form of the tail itself and from the lack of knowledge about the effect of RPA and $2 p 2 h$ in this region. Here, a $100 \%$ uncertainty is applied in the phase space present for $v_{e}$ but absent for $v_{\mu}$ (labeled $v_{e}$ phase space (PS)).

The complete set of interaction model uncertainties includes GENIE implemented uncertainties (Tables 1 and 2), and new uncertainties developed for this effort (Table 4) which represent uncertainties beyond those implemented in the GENIE generator.

Tunes which are applied to the default model, using the dials described, which represent known deficiencies in GENIE's description of neutrino data, are listed in Table 3.

The way model parameters are treated in the analysis is described by three categories:

- Category 1: expected to be constrained with on-axis data; uncertainties are implemented in the same way for ND and FD.

- Category 2: implemented in the same way for ND and FD, but on-axis ND data alone is not sufficient to constrain these parameters. They may be constrained by additional ND samples in future analyses, such as off-axis measurements.

- Category 3: implemented only in the FD. Examples are parameters which only affect $v_{e}$ and $\bar{v}_{e}$ rates which are small and difficult to precisely isolate from background at the ND.

All GENIE uncertainties (original or modified), given in Tables 1 and 2, are all treated as Category 1 . Table 4, which describes the uncertainties beyond those available within GENIE, includes a column identifying which of these categories describes the treatment of each additional uncertainty. 
Table 1 Neutrino interaction cross-section systematic parameters considered in GENIE. GENIE default central values and uncertainties are used for all parameters except the $\mathrm{CC}$ resonance axial mass. The central values are the GENIE nominals, and the $1 \sigma$ uncertainty is as given. Missing GENIE parameters were omitted where uncertainties developed for this analysis significantly overlap with the supplied GENIE freedom, the response calculation was too slow, or the variations were deemed unphysical

\begin{tabular}{ll}
\hline Description & $1 \sigma$ \\
\hline Quasielastic & \\
$M_{\mathrm{A}}^{\mathrm{QE}}$, Axial mass for CCQE & ${ }_{-0.15}^{+0.25} \mathrm{GeV}$ \\
$\mathrm{QE}$ FF, CCQE vector form factor shape & $\mathrm{N} / \mathrm{A}$ \\
$p_{\mathrm{F}}$ Fermi surface momentum for Pauli blocking & $\pm 30 \%$ \\
Low W & \\
$M_{\mathrm{A}}^{\mathrm{RES}}$, Axial mass for CC resonance & $\pm 0.05 \mathrm{GeV}$ \\
$M_{\mathrm{V}}^{\mathrm{RES}}$ Vector mass for CC resonance & $\pm 10 \%$ \\
$\Delta$-decay ang., $\theta_{\pi}$ from $\Delta$ decay (isotropic $\left.\rightarrow \mathrm{R}-\mathrm{S}\right)$ & $\mathrm{N} / \mathrm{A}$ \\
High W (BY model) & \\
$A_{\mathrm{HT}}$, higher-twist in scaling variable $\xi_{w}$ & $\pm 25 \%$ \\
$B_{\mathrm{HT}}$, higher-twist in scaling variable $\xi_{w}$ & $\pm 25 \%$ \\
$C_{\mathrm{V} 1 \mathrm{u}}$, valence GRV98 PDF correction & $\pm 30 \%$ \\
$C_{\mathrm{V} 2 \mathrm{u}}$, valence GRV98 PDF correction & $\pm 40 \%$ \\
Other neutral current & \\
$M_{\mathrm{A}}^{\mathrm{NCRES}}$, Axial mass for NC resonance & $\pm 10 \%$ \\
$M_{\mathrm{V}}^{\mathrm{NCRES}}$, Vector mass for NC resonance & $\pm 5 \%$ \\
\hline
\end{tabular}

Table 2 The intra-nuclear hadron transport systematic parameters implemented in GENIE with associated uncertainties considered in this work. Note that the 'mean free path' parameters are omitted for both $\mathrm{N}-\mathrm{N}$ and $\pi-\mathrm{N}$ interactions as they produced unphysical variations in observable analysis variables. Table adapted from Ref [28]

\begin{tabular}{ll}
\hline Description & $1 \sigma(\%)$ \\
\hline N. CEX, nucleon charge exchange probability & \pm 50 \\
N. EL, nucleon elastic reaction probability & \pm 30 \\
N. INEL, nucleon inelastic reaction probability & \pm 40 \\
N. ABS, nucleon absorption probability & \pm 20 \\
N. PROD, nucleon $\pi$-production probability & \pm 20 \\
$\pi$ CEX, $\pi$ charge exchange probability & \pm 50 \\
$\pi$ EL, $\pi$ elastic reaction probability & \pm 10 \\
$\pi$ INEL, $\pi$ inelastic reaction probability & \pm 40 \\
$\pi$ ABS, $\pi$ absorption probability & \pm 20 \\
$\pi$ PROD, $\pi \pi$-production probability & \pm 20 \\
\hline
\end{tabular}

\section{The near detector simulation and reconstruction}

The ND hall will be located at Fermi National Accelerator Laboratory (Fermilab), $574 \mathrm{~m}$ from where the protons hit the beam target, and $60 \mathrm{~m}$ underground. The baseline design for the DUNE ND system consists of a LArTPC with a downstream magnetized multi-purpose detector (MPD), and
Table 3 Neutrino interaction cross-section systematic parameters that receive a central-value tune and modify the nominal event rate predictions

\begin{tabular}{ll}
\hline Description & Value \\
\hline Quasielastic & \\
BeRPA & $A: 0.59$ \\
$A$ controls low $Q^{2}$ & $B: 1.05$ \\
$B$ controls low-mid $Q^{2}$ & $D: 1.13$ \\
$D$ controls mid $Q^{2}$ & $E: 0.88$ \\
$E$ controls high $Q^{2}$ fall-off & $U: 1.20$ \\
$U$ controls transition from polynomial to exponential & \\
$2 p 2 h$ & \\
$q_{0}, q_{3}$ dependent correction to $2 p 2 h$ events & \\
Low W single pion production & 0.94 \\
Axial mass for CC resonance in GENIE & 0.43 \\
Normalization of CC1 $\pi$ non-resonant interaction & \\
\hline
\end{tabular}

Table 4 List of extra interaction model uncertainties in addition to those provided by GENIE, and the category to which they belong in the analysis. Note that in this analysis, the NC norm. systematic is not applied at the FD, as described in the text

\begin{tabular}{lll}
\hline Uncertainty & Mode & Category \\
\hline BeRPA $[A, B, D]$ & $1 p 1 h / \mathrm{QE}$ & 1 \\
$\operatorname{ArC} 2 p 2 h[v, \bar{\nu}]$ & $2 p 2 h$ & 1 \\
$E_{2 p 2 h}[\mathrm{~A}, \mathrm{~B}][v, \bar{\nu}]$ & $2 p 2 h$ & 2 \\
$\mathrm{NR}[v, \bar{\nu}][\mathrm{CC}, \mathrm{NC}][\mathrm{n}, \mathrm{p}][1 \pi, 2 \pi, 3 \pi]$ & Non-res. pion & 1 \\
$v_{e} \mathrm{PS}$ & $v_{e}, \bar{v}_{e}$ inclusive & 3 \\
$v_{e} / \bar{v}_{e}$ norm & $v_{e}, \bar{v}_{e}$ inclusive & 3 \\
$\mathrm{NC}$ norm. & $\mathrm{NC}$ & $2 *$ \\
\hline
\end{tabular}

an on-axis beam monitor. Additionally, it is planned for the LArTPC and MPD to be movable perpendicular to the beam axis, to take measurements at a number of off-axis angles. The use of off-axis angles is complementary to the on-axis analysis described in this work through the DUNE-PRISM concept, originally developed in the context of the J-PARC neutrino beamline in Ref. [63]. We note that there are many possible ND samples which are not included in the current analysis, but which may either help improve the sensitivity in future, or will help control uncertainties to the level assumed here. These include: neutrino-electron scattering studies, which can independently constrain the flux normalization to $\sim 2 \%$ [64]; additional flux constraints from the low- $v$ method, which exploits the fact that the low energy transfer (low- $v$ ) cross section is roughly constant with neutrino energy [6570]; and using interactions on the gaseous argon (GAr) in the MPD. There is also the potential to include events where the muon does not pass through the MPD, e.g. using multiple Coulomb scattering to estimate the muon momentum [71]. 
The LArTPC is a modular detector based on the ArgonCube design [72], with fully-3D pixelated readout [73] and optical segmentation [74]. These features greatly reduce reconstruction ambiguities that hamper monolithic, projective-readout time projection chambers (TPCs), and enable the ND to function in the high-intensity environment of the DUNE ND site. Each module is itself a LArTPC with two anode planes and a shared central cathode. The active dimensions of each module are $1 \times 3 \times 1 \mathrm{~m}(x \times y \times z)$, where the $z$ direction is along the neutrino beam axis, and the $y$ direction points upward. Charge drifts in the $\pm x$ direction, with a maximum drift distance of $50 \mathrm{~cm}$ for ionization electrons. The full liquid argon (LAr) detector consists of an array of modules in a single cryostat. The minimum active size required for full containment of hadronic showers in the LBNF beam is $3 \times 4 \times 5 \mathrm{~m}$. High-angle muons can also be contained by extending the width to $7 \mathrm{~m}$. For this analysis, 35 modules are arranged in an array 5 modules deep in the $z$ direction and 7 modules across in $x$ so that the total active dimensions are $7 \times 3 \times 5 \mathrm{~m}$. The total active LAr volume is $105 \mathrm{~m}^{3}$, corresponding to a mass of 147 tons.

The MPD used in the analysis consists of a highpressure gaseous argon time-projection chamber (GArTPC) in a cylindrical pressure vessel at 10 bar, surrounded by a granular, high-performance electromagnetic calorimeter, which sits immediately downstream of the LAr cryostat. The pressure vessel is $5 \mathrm{~m}$ in diameter and $5 \mathrm{~m}$ long. The TPC is divided into two drift regions by a central cathode, and filled with a 90\%:10\% $\mathrm{Ar}^{\mathrm{C}} \mathrm{CH}_{4}$ gas mixture, such that $97 \%$ of neutrino interactions will occur on the Ar target. The GArTPC is described in detail in Ref. [5]. The electromagnetic calorimeter (ECAL) is composed of a series of absorber layers followed by arrays of scintillator and is described in Ref. [75]. The entire MPD sits inside a magnetic field, which allows the MPD to precisely measure the momentum and discriminate the sign of particles passing through it.

Neutrino interactions are simulated in the active volume of the LArTPC. The propagation of neutrino interaction products through the LArTPC and MPD detector volumes is simulated using a Geant4-based model [23]. Pattern recognition and reconstruction software has not yet been developed for the ND. Instead, we perform a parameterized reconstruction based on true energy deposits in active detector volumes as simulated by Geant 4 .

The analysis described here uses events originating in the LAr component, within a a fiducial volume (FV) that excludes $50 \mathrm{~cm}$ from the sides and upstream edge, and 150 $\mathrm{cm}$ from the downstream edge of the active region, for a total of $6 \times 2 \times 3 \mathrm{~m}^{2}$. Muons with kinetic energy greater than $\sim 1 \mathrm{GeV}$ typically exit the LAr. An energetic forward-going muon will pass through the ECAL and into the gaseous TPC, where its momentum and charge are reconstructed by curva- ture. For these events, it is possible to differentiate between $\mu^{+}$and $\mu^{-}$event by event. Muons that stop in the LAr or ECAL are reconstructed by range. Events with wide-angle muons that exit the LAr and do not match to the GArTPC are rejected, as the muon momentum is not reconstructed. The asymmetric transverse dimensions of the LAr volume make it possible to reconstruct wide-angle muons with some efficiency.

The charge of muons stopping in the LAr volume cannot be determined event by event. However, the wrong-sign flux is predominantly concentrated in the high-energy tail, where leptons are more likely to be forward and energetic. In FHC beam running, the wrong-sign background in the focusing peak is negligibly small, and $\mu^{-}$is assumed for all stopping muon tracks. In RHC beam running, the wrong-sign background is larger in the peak region. Furthermore, high-angle leptons are generally at higher inelasticity, which enhances the wrong-sign contamination in the contained muon subsample. To mitigate this, a Michel electron is required at the end of the track. The wrong-sign $\mu^{-}$captures on Ar with $75 \%$ probability, effectively suppressing the relative $\mu^{-}$component by a factor of four.

True muons and charged pions are evaluated as potential muon candidates. The track length is determined by following the true particle trajectory until it undergoes a hard scatter or ranges out. The particle is classified as a muon if its track length is at least $1 \mathrm{~m}$, and the mean energy deposit per centimeter of track length is less than $3 \mathrm{MeV}$. The mean energy cut rejects tracks with detectable hadronic interactions. The minimum length requirement imposes an effective threshold on true muons of about $200 \mathrm{MeV}$ kinetic energy, but greatly suppresses potential NC backgrounds with low-energy, non-interacting charged pions. Chargedcurrent events are required to have exactly one muon, and if the charge is reconstructed, it must be of the appropriate charge.

As in the FD reconstruction described in Sect. 5, hadronic energy in the ND is reconstructed by summing all charge deposits in the LAr active volume that are not associated with the muon. To reject events where the hadronic energy is poorly reconstructed due to particles exiting the detector, a veto region is defined as the outer $30 \mathrm{~cm}$ of the active volume on all sides. Events with more than $30 \mathrm{MeV}$ total energy deposit in the veto region are excluded from the analysis. This leads to an acceptance that decreases as a function of hadronic energy, as shown in the bottom panel of Fig. 5. Neutron energy is typically not observed, resulting in poor reconstruction of events with energetic neutrons, as well as in events where neutrons are produced in secondary interactions inside the detector. The reconstructed neutrino energy is the sum of the reconstructed hadronic energy and the reconstructed muon energy. 

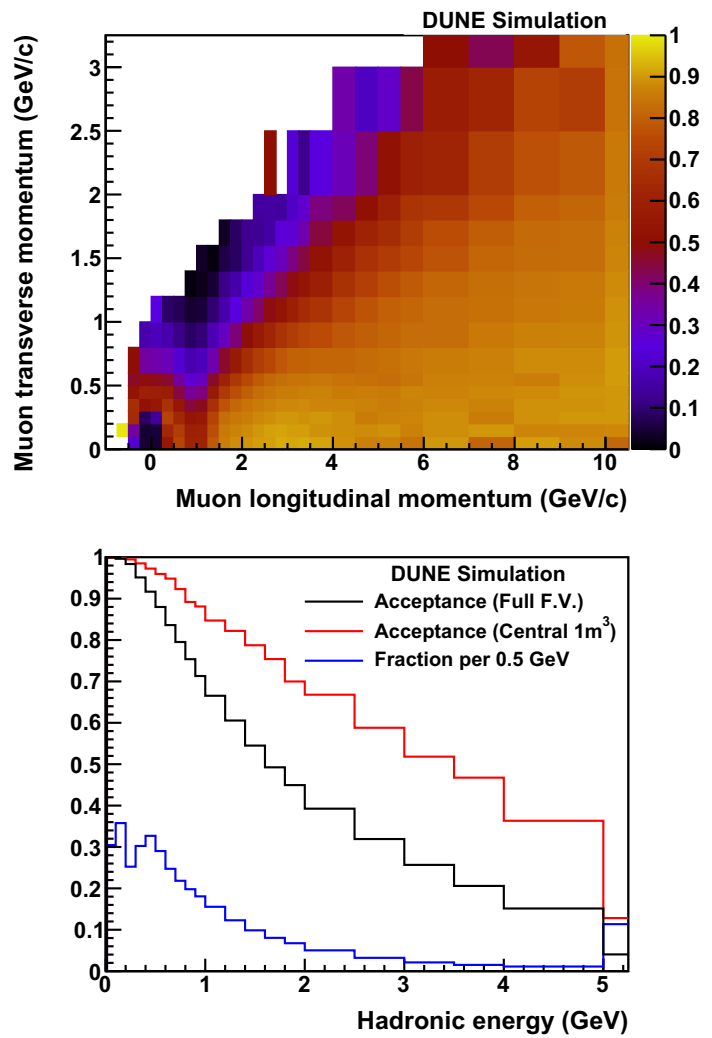

Fig. 5 Top: LAr+MPD acceptance for $v_{\mu} \mathrm{CC}$ events as a function of muon transverse and longitudinal momentum. Bottom: Acceptance as a function of hadronic energy; the black line is for the full Fiducial Volume $(\mathrm{FV})$ while the red line is for a $1 \times 1 \times 1 \mathrm{~m}^{3}$ volume in the center, where the acceptance is higher due to the better hadron containment. The blue curve shows the expected distribution of true hadronic energy in the DUNE ND flux normalized to unity; $56 \%$ of events have hadronic energy below $1 \mathrm{GeV}$ where the acceptance is high

The oscillation analysis presented here includes samples of $v_{\mu}$ and $\bar{v}_{\mu}$ charged-current interactions originating in the LAr portion of the ND, as shown in Fig. 6. These samples are binned in two dimensions as a function of reconstructed neutrino energy and inelasticity, $y_{\mathrm{rec}}=1-E_{\mu}^{\mathrm{rec}} / E_{\nu}^{\mathrm{rec}}$, where $E_{\mu}^{\mathrm{rec}}$ and $E_{\nu}^{\mathrm{rec}}$ are the reconstructed muon and neutrino energies, respectively. Backgrounds to $\bar{\nu}_{\mu}^{\prime} \mathrm{CC}$ arise from $\mathrm{NC} \pi^{ \pm}$ production where the pion leaves a long track and does not shower. Muons below about $400 \mathrm{MeV}$ kinetic energy have a significant background from charged pions, so these CC events are excluded from the selected sample. Wrong-sign contamination in the beam is an additional background, particularly at low reconstructed neutrino energies in RHC.

\section{The far detector simulation and reconstruction}

The 40-kt DUNE FD consists of four separate LArTPC detector modules, each with a FV of at least $10 \mathrm{kt}$, installed $\sim 1.5 \mathrm{~km}$ underground at the Sanford Underground Research
Facility (SURF) [76]. DUNE is committed to deploying both single-phase [77] and dual-phase [78] LArTPC technologies, and is investigating advanced detector designs for the fourth detector module. As such, the exact order of construction and number of modules of each design is unknown. In this work, the FD reconstruction performance is assessed assuming a single-phase design for all four modules, which does not fully exploit the benefits of different technologies with independent systematics in the sensitivity studies. A full simulation chain has been developed, from the generation of neutrino events in a Geant 4 model of the FD geometry, to efficiencies and reconstructed neutrino energy estimators of all samples used in the sensitivity analysis.

The total active LAr volume of each single-phase DUNE FD detector module is $13.9 \mathrm{~m}$ long, $12.0 \mathrm{~m}$ high and $13.3 \mathrm{~m}$ wide, with the $13.3 \mathrm{~m}$ width in the drift direction subdivided into four independent drift regions, with two shared cathodes. Full details of the single-phase detector module design can be found in Ref. [79]. The total active volume of each module is $\sim 13 \mathrm{kt}$, the FV of at least $10 \mathrm{kt}$ is defined by studies of neutrino energy resolution, using the neutrino energy estimators described below. At the anode, there are two wrapped-wire readout induction planes, which are offset by $\pm 35.7^{\circ}$ to the vertical, and a vertical collection plane.

Neutrino interactions of all flavors are simulated such that weights can be applied to produce samples for any set of oscillation parameters. The interaction model described in Sect. 3 was used to model the neutrino-argon interactions in the volume of the cryostat, and the final-state particles are propagated in the detector through Geant 4 . The electronics response to the ionization electrons and scintillation light is simulated to produce digitized signals in the wire planes and photon detectors (PDs) respectively.

Raw detector signals are processed using algorithms to remove the impact of the LArTPC electric field and electronics response from the measured signal, to identify hits, and to cluster hits that may be grouped together due to proximity in time and space. Clusters from different wire planes are matched to form high-level objects such as tracks and showers. These high level objects are used as inputs to the neutrino energy reconstruction algorithm.

The energy of the incoming neutrino in CC events is estimated by adding the lepton and hadronic energies reconstructed using the Pandora toolkit $[80,81]$. If the event is selected as $v_{\mu} \mathrm{CC}$, the neutrino energy is estimated as the sum of the energy of the longest reconstructed track and the hadronic energy. The energy of the longest reconstructed track is estimated from its range if the track is contained in the detector. If the longest track exits the detector, its energy is estimated from multiple Coulomb scattering. The hadronic energy is estimated from the charge of reconstructed hits that are not in the longest track, and corrections are applied to each hit charge for recombination and the electron lifetime. 


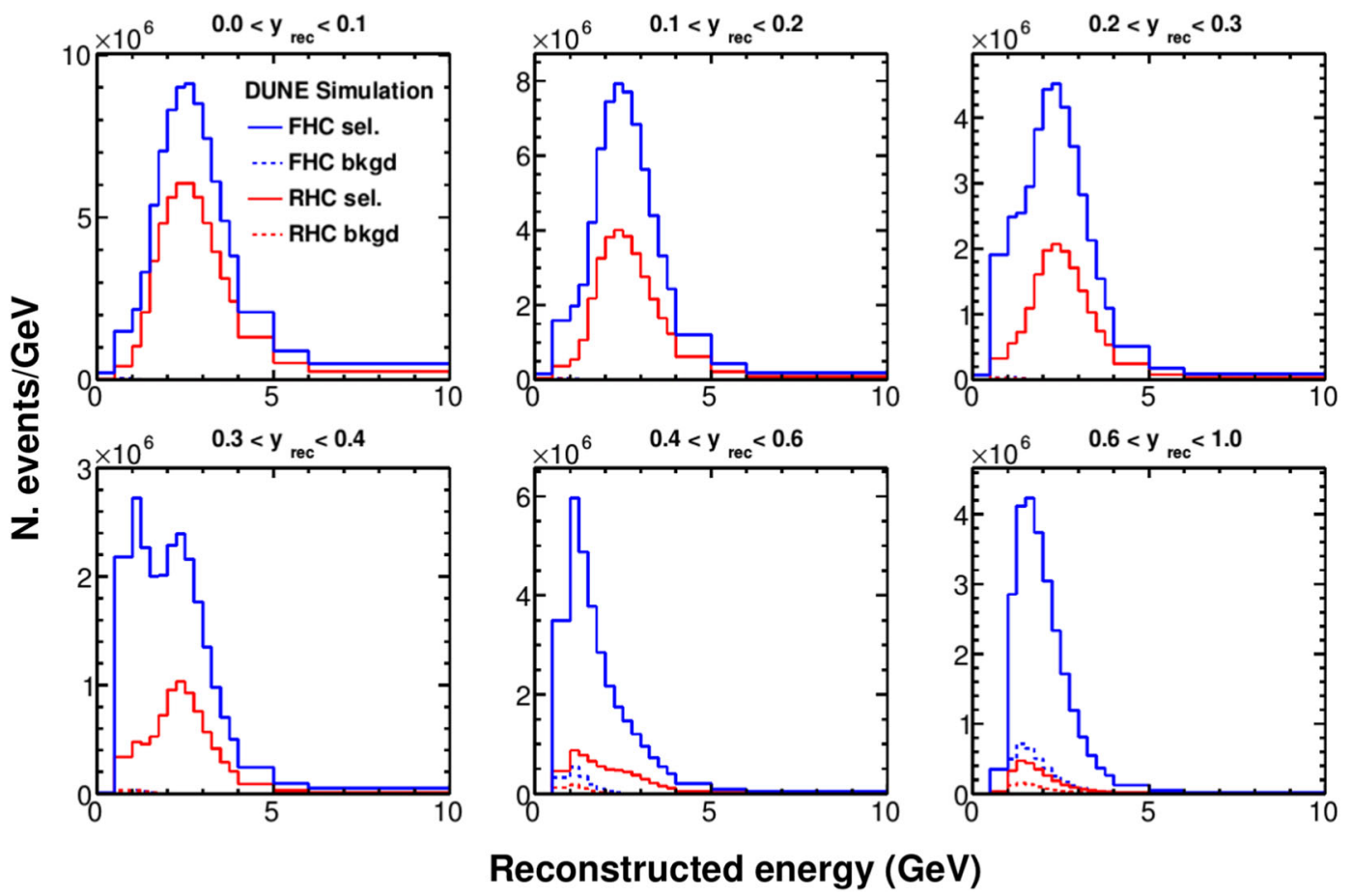

Fig. 6 ND samples in both FHC (blue) and RHC (red), shown in the reconstructed neutrino energy and reconstructed inelasticity binning used in the analysis, shown for a 7 year staged exposure, with an equal

An additional correction is made to the hadronic energy to account for missing energy due to neutral particles and finalstate interactions.

If the event is selected as $v_{e} \mathrm{CC}$, the energy of the neutrino is estimated as the sum of the energy of the reconstructed electromagnetic (EM) shower with the highest energy and the hadronic energy. The former is estimated from the charges of the reconstructed hits in the shower, and the latter from the hits not in the shower; the recombination and electron lifetime corrections are applied to the charge of each hit. The same hadronic shower energy calibration is used for both $v$ and $\bar{v}$ based on a sample of $v$ and $\bar{v}$ events.

In the energy range of $0.5-4 \mathrm{GeV}$ that is relevant for oscillation measurements, the observed neutrino energy resolution is $\sim 15-20 \%$, depending on lepton flavor and reconstruction method. The muon energy resolution is $4 \%$ for contained tracks and $18 \%$ for exiting tracks. The electron energy resolution is approximately $4 \% \oplus 9 \% / \sqrt{E}$, with some shower leakage that gives rise to a non-Gaussian tail that is anticorrelated with the hadronic energy measurement. The hadronic energy resolution is $34 \%$, which could be further improved by identifying individual hadrons, adding masses of charged pions, and applying particle-specific recombination corrections. It may also be possible to identify final-state neutrons by looking for neutron-nucleus scatters, and use event kinematics to split between FHC and RHC. Backgrounds are also shown (dashed lines), which are dominated by NC events, although there is some contribution from wrong-sign $v_{\mu}$ background events in $\mathrm{RHC}$

further inform the energy estimate. These improvements are under investigation and are not included in this analysis.

Event classification is carried out through image recognition techniques using a convolutional neural network, named convolutional visual network (CVN). Detailed descriptions of the CVN architecture can be found in Ref. [82]. The primary goal of the $\mathrm{CVN}$ is to efficiently and accurately produce event selections of the following interactions: $v_{\mu} \mathrm{CC}$ and $v_{e}$ $\mathrm{CC}$ in $\mathrm{FHC}$, and $\bar{v}_{\mu} \mathrm{CC}$ and $\bar{v}_{e} \mathrm{CC}$ in RHC.

In order to build the training input to the DUNE CVN three images of the neutrino interactions are produced, one for each of the three readout views of the LArTPC, using the reconstructed hits on individual wire planes. Each pixel contains information about the integrated charge in that region. An example of a simulated $2.2 \mathrm{GeV} v_{e} \mathrm{CC}$ interaction is shown in a single view in Fig. 7 demonstrating the fine-grained detail available from the LArTPC technology.

The CVN is trained using approximately three million simulated neutrino interactions. A statistically independent sample is used to generate the physics measurement sensitivities. The training sample is chosen to ensure similar numbers of training examples from the different neutrino flavors. Validation is performed to ensure that similar classification performance is obtained for the training and test samples to ensure that the CVN is not overtrained. 


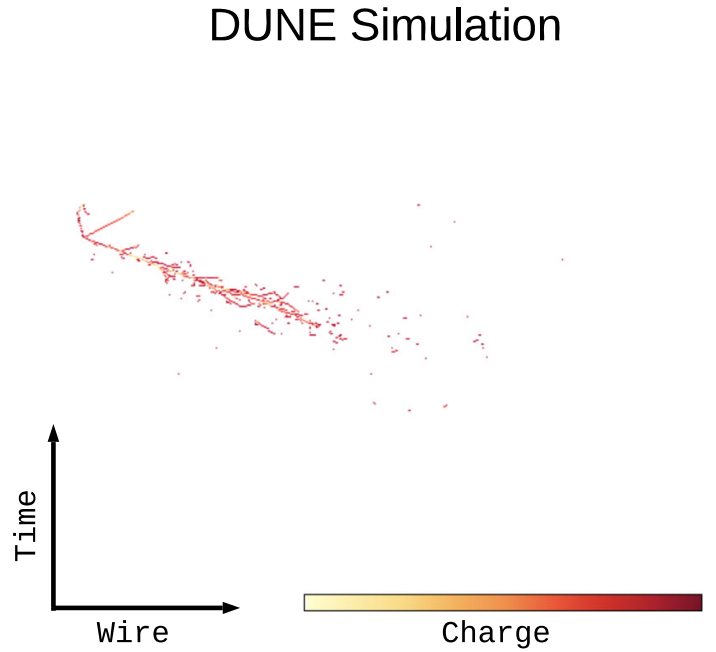

Fig. 7 A simulated $2.2 \mathrm{GeV} v_{e} \mathrm{CC}$ interaction shown in the collection view of the DUNE LArTPCs. The horizontal axis shows the wire number of the readout plane and the vertical axis shows time. The colorscale shows the charge of the energy deposits on the wires. The interaction looks similar in the other two views. Reproduced from Ref. [82]

For the analysis presented here, we use the CVN score for each interaction to belong to one of the following classes: $v_{\mu} \mathrm{CC}, v_{e} \mathrm{CC}, v_{\tau} \mathrm{CC}$ and NC. The $v_{e}$ CC score distribution, $P\left(v_{e} \mathrm{CC}\right)$, and the $v_{\mu} \mathrm{CC}$ score distribution, $P\left(v_{\mu} \mathrm{CC}\right)$, are shown in Fig. 8. Excellent separation between the signal and background interactions is seen in both cases. The event selection requirement for an interaction to be included in the $v_{e} \mathrm{CC}\left(v_{\mu} \mathrm{CC}\right)$ is $P\left(v_{e} \mathrm{CC}\right)>0.85\left(P\left(v_{\mu} \mathrm{CC}\right)>0.5\right)$, optimized to produce the best sensitivity to charge parity (CP) violation. Since all of the flavor classification scores must sum to unity, the interactions selected in the two event selections are completely independent. The same selection criteria are used for both FHC and RHC beam running.

Figure 9 shows the efficiency as a function of reconstructed energy (under the electron neutrino hypothesis) for the $v_{e}$ event selection, and the corresponding selection efficiency for the $v_{\mu}$ event selection. The $v_{e}$ and $v_{\mu}$ efficiencies in both FHC and RHC beam modes all exceed $90 \%$ in the neutrino flux peak.

The ability of the CVN to identify neutrino flavor is dependent on its ability to resolve and identify the charged lepton. Backgrounds originate from the mis-identification of charged pions for $v_{\mu}$ disappearance, and photons for $v_{e}$ appearance. The probability for these backgrounds to be introduced varies with the momentum and isolation of the energy depositions from the pions and photons. The efficiency was also observed to drop as a function of track/shower angle (with respect to the incoming neutrino beam direction) when energy depositions aligned with wire planes. The shapes of the efficiency functions in lepton momentum, lepton angle, and hadronic energy fraction (inelasticity) are all observed to be consistent
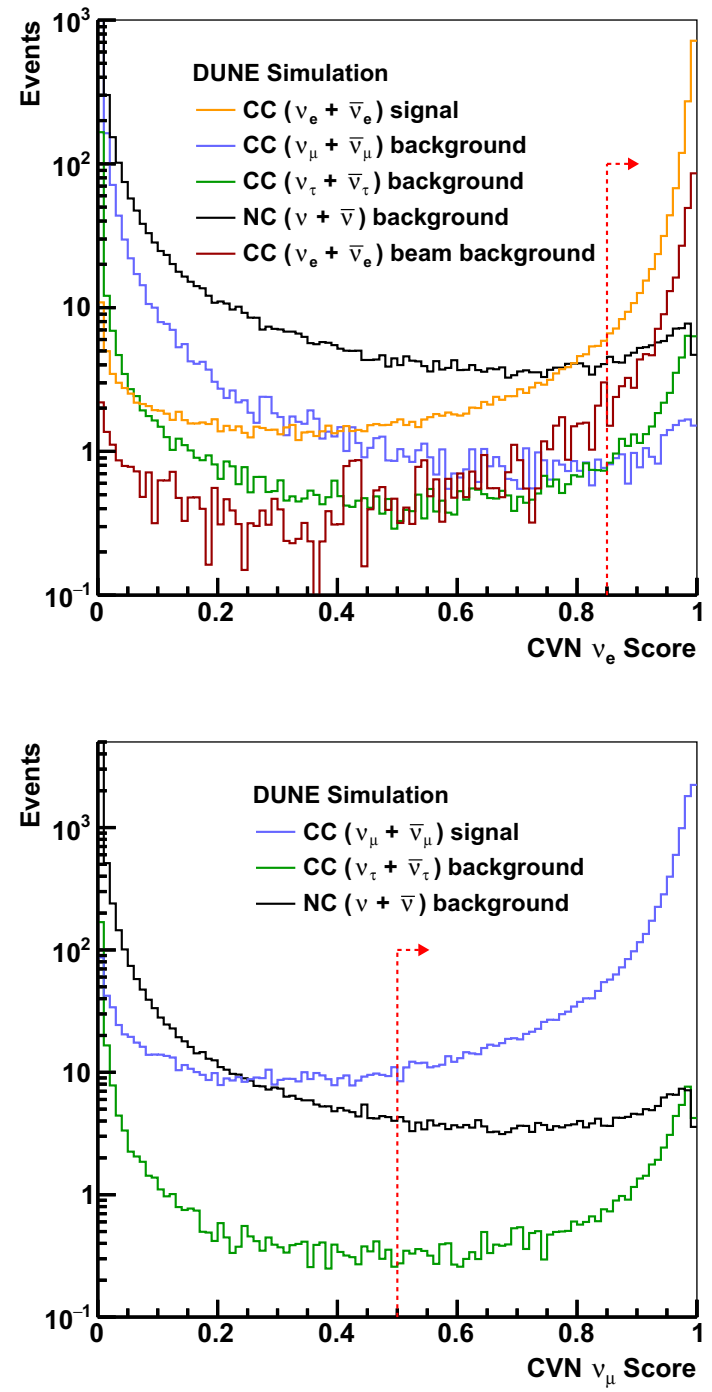

Fig. 8 The distribution of CVN $v_{e} \mathrm{CC}$ (top) and $v_{\mu} \mathrm{CC}$ scores (bottom) for FHC shown with a log scale. Reproduced from Ref. [82]

with results from previous studies, including hand scans of LArTPC simulations. The CVN is susceptible to bias if there are features in the data that are not present in the simulation, so before its use on data, it will be important to comprehensively demonstrate that the selection is not sensitive to the choice of reference models. A discussion of the bias studies performed so far, and those planned in future, can be found in Ref. [82].

\section{Expected far detector event rate and oscillation parameters}

In this work, FD event rates are calculated assuming the following nominal deployment plan, which is based on a technically limited schedule: 

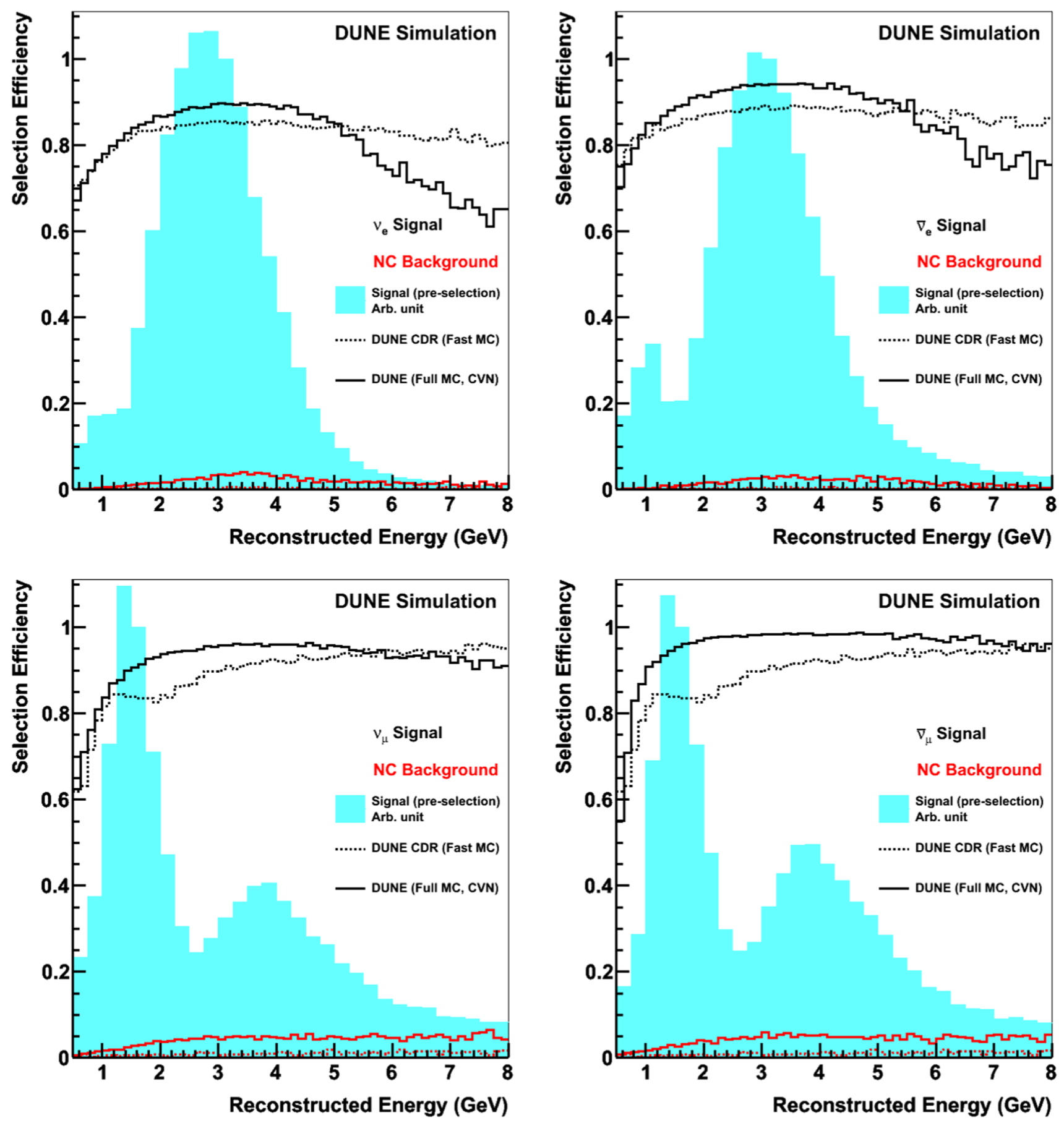

Fig. 9 Top: the $v_{e} \mathrm{CC}$ selection efficiency for FHC (left) and RHC (right) simulation with the criterion $P\left(v_{e} \mathrm{CC}\right)>0.85$. Bottom: the $v_{\mu}$ CC selection efficiency for FHC (left) and RHC (right) simulation with the criterion $P\left(v_{\mu} \mathrm{CC}\right)>0.5$. The results from DUNE's Conceptual Design Report (CDR) are shown for comparison [7]. The solid (dashed)

lines show results from the CVN (CDR) for signal $v_{e} \mathrm{CC}$ and $\bar{v}_{e} \mathrm{CC}$ events in black and $\mathrm{NC}$ background interaction in red. The blue region shows the oscillated flux (A.U.) to illustrate the most important regions of the energy distribution. Reproduced from Ref. [82]

- Start of beam run: two FD module volumes for total fiducial mass of $20 \mathrm{kt}, 1.2 \mathrm{MW}$ beam

- After one year: add one FD module volume for total fiducial mass of $30 \mathrm{kt}$

- After three years: add one FD module volume for total fiducial mass of $40 \mathrm{kt}$

- After 6 years: upgrade to $2.4 \mathrm{MW}$ beam 
Table 5 Conversion between number of years in the nominal staging plan, and kt-MW-years, the two quantities used to indicate exposure in this analysis

\begin{tabular}{ll}
\hline Years & kt-MW-years \\
\hline 7 & 336 \\
10 & 624 \\
15 & 1104 \\
\hline
\end{tabular}

Table 5 shows the conversion between number of years under the nominal staging plan, and kt-MW-years, which are used to indicate the exposure in this analysis. For all studies shown in this work, a 50\%/50\% ratio of FHC to RHC data was assumed, based on studies which showed a roughly equal mix of running produced a nearly optimal $\delta_{\mathrm{CP}}$ and mass ordering sensitivity. The exact details of the run plan are not included in the staging plan.

Event rates are calculated with the assumption of 1.1 $\times 10^{21}$ protons on target (POT) per year, which assumes a combined uptime and efficiency of the Fermilab accelerator accelerator complex and the LBNF beamline of 57\% [5].

Figures 10 and 11 show the expected rate of selected events for $v_{e}$ appearance and $v_{\mu}$ disappearance, respectively, including expected flux, cross section, and oscillation probabilities, as a function of reconstructed neutrino energy at a baseline of $1285 \mathrm{~km}$. The spectra are shown for a 3.5 year (staged) exposure each for FHC and RHC beam modes, for a total run time of seven years. The rates shown are scaled to obtain different exposures. Tables 6 and 7 give the integrated rate for the $v_{e}$ appearance and $v_{\mu}$ disappearance spectra, respectively. Note that the total rates are integrated over the range of reconstructed neutrino energies used in the analysis, 0.5$10 \mathrm{GeV}$. The nominal neutrino oscillation parameters used in Figs. 10 and 11 and the uncertainty on those parameters (used later in the analysis) are taken from the NuFIT $[9,83]$ global fit to neutrino data, and their values are given in Table 8. See also Refs. [10] and [11] for other recent global fits.

As can be seen in Fig. 10, the background to $v_{e}$ appearance is composed of: (1) CC interactions of $\nu_{e}$ and $\bar{v}_{e}$ intrinsic to the beam; (2) misidentified NC interactions; (3) misidentified $v_{\mu}$ and $\bar{v}_{\mu}$ CC interactions; and (4) $v_{\tau}$ and $\bar{v}_{\tau}$ CC interactions in which the $\tau$ 's decay leptonically into electrons/positrons. $\mathrm{NC}$ and $\nu_{\tau}$ backgrounds emanate from interactions of higherenergy neutrinos that feed down to lower reconstructed neutrino energies due to missing energy in unreconstructed finalstate neutrinos. The selected $\mathrm{NC}$ and $\mathrm{CC} v_{\mu}$ generally include an asymmetric decay of a relatively high energy $\pi^{0}$ coupled with a prompt photon conversion. As can be seen in Fig. 11, the backgrounds to the $v_{\mu}$ disappearance are due to wrong-sign $v_{\mu}$ interactions, which cannot easily be distinguished in the unmagnetized DUNE FD, and NC interactions, where a pion has been misidentified as the primary muon. As expected, the $v_{\mu}$ background in RHC is much larger than the $\bar{v}_{\mu}$ background in FHC.
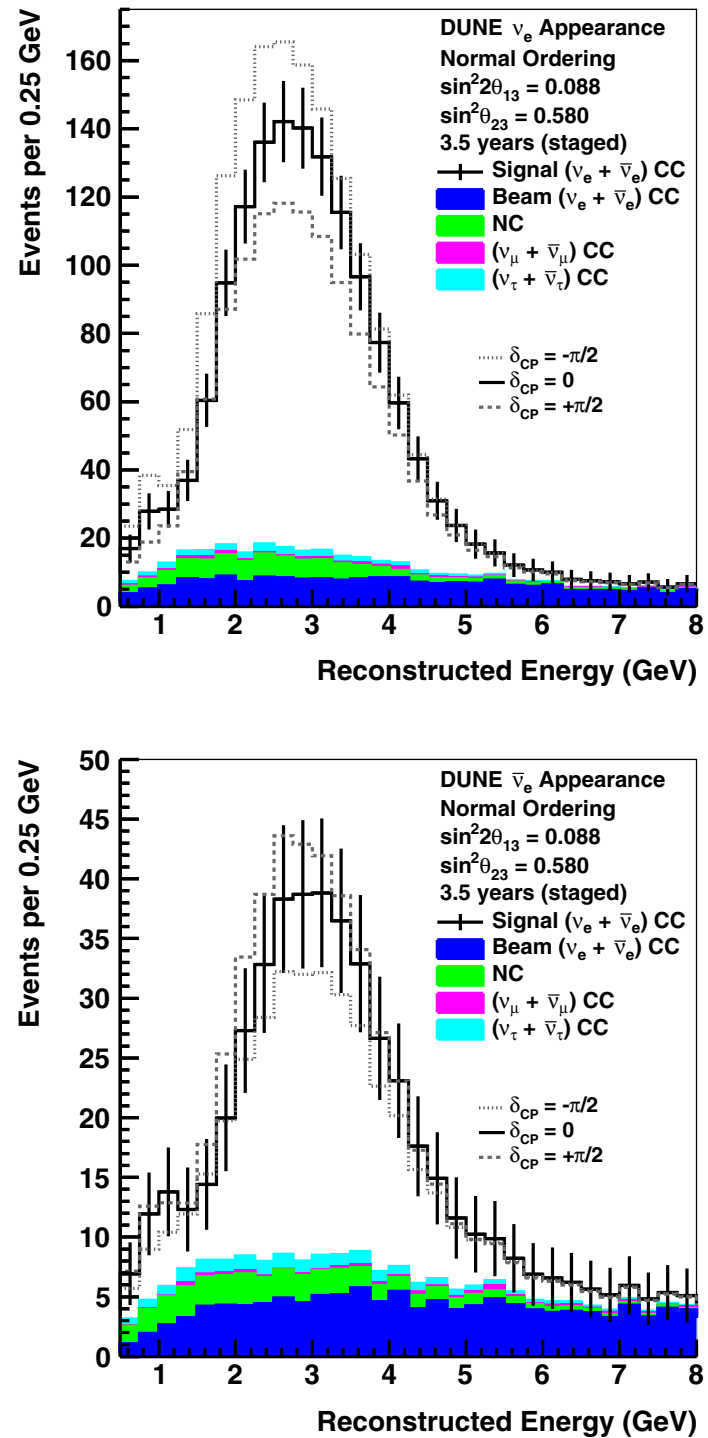

Fig. $10 v_{e}$ and $\bar{v}_{e}$ appearance spectra: reconstructed energy distribution of selected $v_{e}$ CC-like events assuming 3.5 years (staged) running in the neutrino-beam mode (top) and antineutrino-beam mode (bottom), for a total of seven years (staged) exposure. Statistical uncertainties are shown on the datapoints. The plots assume normal mass ordering and include curves for $\delta_{\mathrm{CP}}=-\pi / 2,0$, and $\pi / 2$

\section{Detector uncertainties}

Detector effects impact the event selection efficiency as well as the reconstruction of quantities used in the oscillation fit, such as neutrino energy. The main sources of detector systematic uncertainties are limitations of the expected calibration and modeling of particles in the detector.

The ND LArTPC uses similar technology to the FD, but important differences lead to uncertainties that do not fully correlate between the two detectors. First, the readout technology is different, as the ND LArTPC uses pixels as well as a different, modular photon detector. Therefore, the charge 

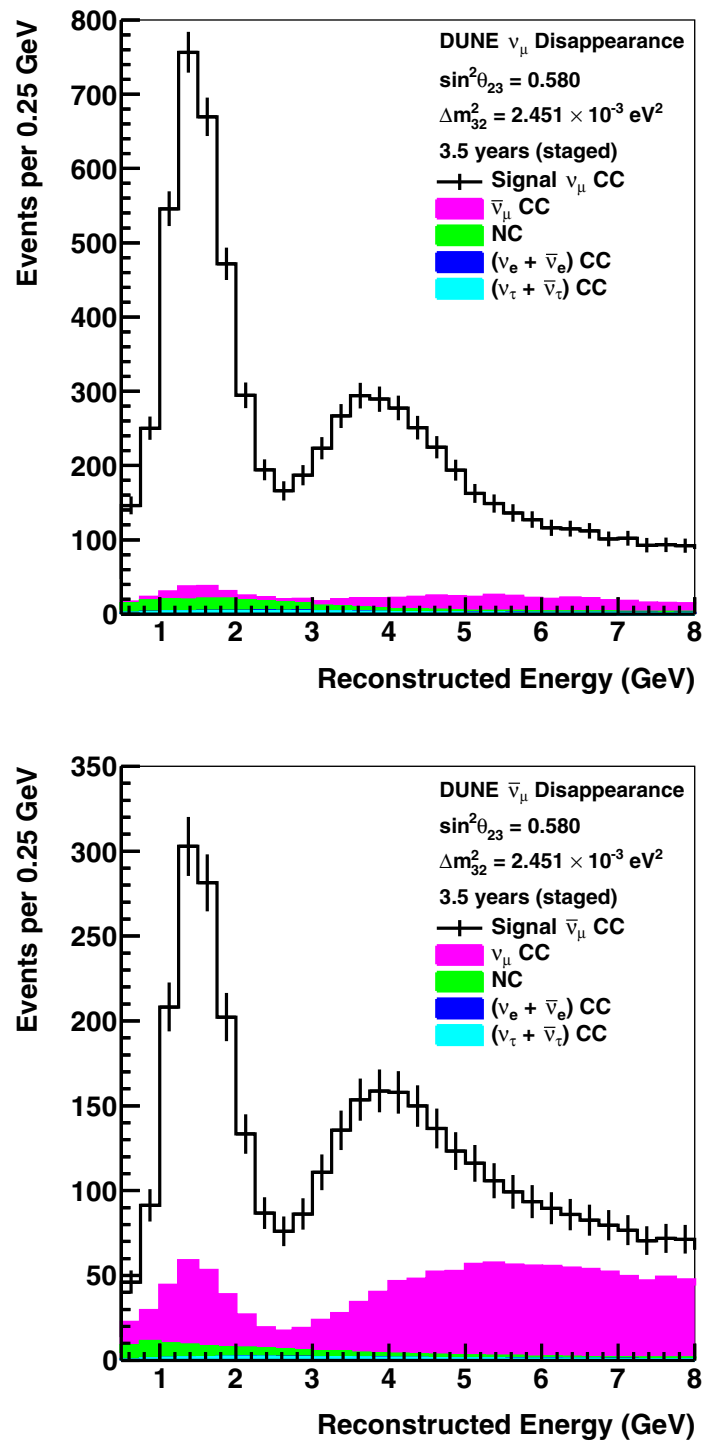

Fig. $11 v_{\mu}$ and $\bar{v}_{\mu}$ disappearance spectra: reconstructed energy distribution of selected $v_{\mu}$ CC-like events assuming 3.5 years (staged) running in the neutrino-beam mode (top) and antineutrino-beam mode (bottom), for a total of seven years (staged) exposure. Statistical uncertainties are shown on the datapoints. The plots assume normal mass ordering

response will be different between near and far detectors due to differences in electronics readout, noise, and local effects like alignment. Second, the high-intensity environment of the ND complicates associating detached energy deposits to events, a problem which is not present in the FD. Third, the calibration strategies will be different. For example, the ND has a high-statistics calibration sample of through-going, momentum-analyzed muons from neutrino interactions in the upstream rock, which is not available with high statistics for the FD. Finally, the reconstruction efficiency will be inherently different due to the relatively small size of the ND. Containment of charged hadrons will be significantly worse at the
Table $6 v_{e}$ and $\bar{v}_{e}$ appearance rates: integrated rate of selected $v_{e} \mathrm{CC}$ like events between 0.5 and $10.0 \mathrm{GeV}$ assuming a 3.5-year (staged) exposure in the neutrino-beam mode and antineutrino-beam mode. The rates are shown for both $\mathrm{NO}$ and $\mathrm{IO}$, and signal events are shown for both $\delta_{\mathrm{CP}}=0$ and $\delta_{\mathrm{CP}}=-\pi / 2$

\begin{tabular}{|c|c|c|c|c|}
\hline \multirow[t]{3}{*}{ Sample } & \multicolumn{4}{|c|}{ Expected Events } \\
\hline & \multicolumn{2}{|c|}{$\delta_{\mathrm{CP}}=0$} & \multicolumn{2}{|c|}{$\delta_{\mathrm{CP}}=-\frac{\pi}{2}$} \\
\hline & NO & $\mathrm{IO}$ & NO & IO \\
\hline \multicolumn{5}{|l|}{$v$ mode } \\
\hline Oscillated $v_{e}$ & 1155 & 526 & 1395 & 707 \\
\hline Oscillated $\bar{v}_{e}$ & 19 & 33 & 14 & 28 \\
\hline Total oscillated & 1174 & 559 & 1409 & 735 \\
\hline Beam $v_{e}+\bar{v}_{e}$ CC background & 228 & 235 & 228 & 235 \\
\hline NC background & 84 & 84 & 84 & 84 \\
\hline$v_{\tau}+\bar{v}_{\tau} \mathrm{CC}$ background & 36 & 36 & 35 & 36 \\
\hline$v_{\mu}+\bar{v}_{\mu} \mathrm{CC}$ background & 15 & 15 & 15 & 15 \\
\hline Total background & 363 & 370 & 362 & 370 \\
\hline \multicolumn{5}{|l|}{$\bar{v}$ mode } \\
\hline Oscillated $v_{e}$ & 81 & 39 & 95 & 53 \\
\hline Oscillated $\bar{v}_{e}$ & 236 & 492 & 164 & 396 \\
\hline Total oscillated & 317 & 531 & 259 & 449 \\
\hline Beam $v_{e}+\bar{v}_{e}$ CC background & 145 & 144 & 145 & 144 \\
\hline $\mathrm{NC}$ background & 40 & 40 & 40 & 40 \\
\hline$v_{\tau}+\bar{v}_{\tau} \mathrm{CC}$ background & 22 & 22 & 22 & 22 \\
\hline$v_{\mu}+\bar{v}_{\mu}$ CC background & 6 & 6 & 6 & 6 \\
\hline Total background & 216 & 215 & 216 & 215 \\
\hline
\end{tabular}

$\mathrm{ND}$, especially for events with energetic hadronic showers or with vertices near the edges of the FV.

An uncertainty on the overall energy scale is included in the analysis presented here, as well as particle response uncertainties that are separate and uncorrelated between four species: muons, charged hadrons, neutrons, and electromagnetic showers. In the ND, muons reconstructed by range in LAr and by curvature in the MPD are treated separately. The energy scale and particle response uncertainties are allowed to vary with energy; each term is described by three free parameters:

$E_{\text {rec }}^{\prime}=E_{\text {rec }} \times\left(p_{0}+p_{1} \sqrt{E_{\text {rec }}}+\frac{p_{2}}{\sqrt{E_{r e c}}}\right)$

where $E_{r e c}$ is the nominal reconstructed energy, $E_{r e c}^{\prime}$ is the shifted energy, and $p_{0}, p_{1}$, and $p_{2}$ are free fit parameters that are allowed to vary within a priori constraints. Note that the parameters produce a shift to the kinematic variables in an event, as opposed to simply assigning a weight to each simulated event. The energy scale and resolution parameters are conservatively treated as uncorrelated between the ND and FD. With a better understanding of the relationship between ND and FD calibration and reconstruction techniques, it may be possible to correlate some portion of the energy response. 
Table $7 \quad v_{\mu}$ and $\bar{v}_{\mu}$ disappearance rates: integrated rate of selected $v_{\mu}$ CC-like events between 0.5 and $10.0 \mathrm{GeV}$ assuming a 3.5-year (staged) exposure in the neutrino-beam mode and antineutrino-beam mode. The rates are shown for both $\mathrm{NO}$ and $\mathrm{IO}$, with $\delta_{\mathrm{CP}}=0$

\begin{tabular}{lll}
\hline Sample & $\begin{array}{l}\text { Expected Events } \\
\text { NO }\end{array}$ & IO \\
\hline$v$ mode & & \\
$v_{\mu}$ signal & 7235 & 7368 \\
$\bar{v}_{\mu}$ CC background & 542 & 542 \\
NC background & 213 & 213 \\
$v_{\tau}+\bar{v}_{\tau}$ CC background & 53 & 54 \\
$v_{e}+\bar{v}_{e}$ CC background & 9 & 5 \\
$\bar{v}$ mode & & \\
$\bar{v}_{\mu}$ signal & 2656 & 2633 \\
$v_{\mu}$ CC background & 1590 & 1600 \\
NC background & 109 & 109 \\
$v_{\tau}+\bar{v}_{\tau}$ CC background & 31 & 31 \\
$v_{e}+\bar{v}_{e}$ CC background & 2 & 2 \\
\hline
\end{tabular}

Table 8 Central value and relative uncertainty of neutrino oscillation parameters from a global fit $[9,83]$ to neutrino oscillation data. The matter density is taken from Ref. [84]. Because the probability distributions are somewhat non-Gaussian (particularly for $\theta_{23}$ ), the relative uncertainty is computed using $1 / 6$ of the $3 \sigma$ allowed range from the fit, rather than $1 / 2$ of the $1 \sigma$ range. For $\theta_{23}, \theta_{13}$, and $\Delta m_{31}^{2}$, the best-fit values and uncertainties depend on whether normal mass ordering (NO) or inverted mass ordering (IO) is assumed

\begin{tabular}{lll}
\hline Parameter & Central value & Relative uncertainty $(\%)$ \\
\hline$\theta_{12}$ & 0.5903 & 2.3 \\
$\theta_{23}(\mathrm{NO})$ & 0.866 & 4.1 \\
$\theta_{23}(\mathrm{IO})$ & 0.869 & 4.0 \\
$\theta_{13}(\mathrm{NO})$ & 0.150 & 1.5 \\
$\theta_{13}(\mathrm{IO})$ & 0.151 & 1.5 \\
$\Delta m_{21}^{2}$ & $7.39 \times 10^{-5} \mathrm{eV}^{2}$ & 2.8 \\
$\Delta m_{32}^{2}(\mathrm{NO})$ & $2.451 \times 10^{-3} \mathrm{eV}^{2}$ & 1.3 \\
$\Delta m_{32}^{2}(\mathrm{IO})$ & $-2.512 \times 10^{-3} \mathrm{eV}^{2}$ & 1.3 \\
$\rho$ & $2.848 \mathrm{~g} \mathrm{~cm}^{-3}$ & 2 \\
\hline
\end{tabular}

The full list of assumed energy scale uncertainties is given as Table 9. In addition to the uncertainties on the energy scale, uncertainties on energy resolutions are also included. These are treated as fully uncorrelated between the near and far detectors and are taken to be $2 \%$ for muons, charged hadrons, and EM showers and $40 \%$ for neutrons.

The scale of these assumed uncertainties is motivated by what has been achieved in recent experiments, including calorimetric based approaches (NOvA, MINERvA) and LArTPCs (LArIAT, MicroBooNE, ArgoNeuT). The DUNE performance is expected to significantly exceed the performance of these current surface-based experiments. NOvA [44] has achieved $<1 \%$ (5\%) uncertainties on the energy
Table 9 Uncertainties applied to the energy response of various particles. $p_{0}, p_{1}$, and $p_{2}$ correspond to the constant, square root, and inverse square root terms in the energy response parameterization given in Eq. (2). All are treated as uncorrelated between the ND and FD

\begin{tabular}{llll}
\hline Particle type & \multicolumn{3}{l}{ Allowed variation } \\
\cline { 2 - 4 } & $p_{0}(\%)$ & $p_{1}(\%)$ & $p_{2}(\%)$ \\
\hline All (except muons) & 2 & 1 & 2 \\
$\mu$ (range) & 2 & 2 & 2 \\
$\mu$ (curvature) & 1 & 1 & 1 \\
$\mathrm{p}, \pi^{ \pm}$ & 5 & 5 & 5 \\
$\mathrm{e}, \gamma, \pi^{0}$ & 2.5 & 2.5 & 2.5 \\
$\mathrm{n}$ & 20 & 30 & 30 \\
\hline
\end{tabular}

scale of muons (protons). Uncertainties associated to the pion and proton re-interactions in the detector medium are expected to be controlled from ProtoDUNE and LArIAT data, as well as the combined analysis of low density (gaseous) and high density (LAr) NDs. Uncertainties in the E field also contribute to the energy scale uncertainty [85], and calibration is needed (with cosmics at ND, laser system at FD) to constrain the overall energy scale. The recombination model will continue to be validated by the suite of LAr experiments and is not expected to be an issue for nominal field provided minimal $\mathrm{E}$ field distortions. Uncertainties in the electronics response are controlled with a dedicated charge injection system and validated with intrinsic sources, Michel electrons and ${ }^{39} \mathrm{Ar}$.

The response of the detector to neutrons is a source of active study and will couple strongly to detector technology. The validation of neutron interactions in LAr will continue to be characterized by dedicated measurements (e.g., CAPTAIN [86,87]) and the LAr program (e.g., ArgoNeuT [88]). However, the association of the identification of a neutron scatter or capture to the neutron's true energy has not been demonstrated, and significant reconstruction issues exist, so a large uncertainty (20\%) is assigned comparable to the observations made by MINERvA [89] assuming they are attributed entirely to the detector model. Selection of photon candidates from $\pi^{0}$ is also a significant reconstruction challenge, but a recent measurement from MicroBooNE indicates this is possible and the reconstructed $\pi^{0}$ invariant mass has an uncertainty of 5\%, although with some bias [90].

The $p_{1}$ and $p_{2}$ terms in Eq. (2) allow the energy response to vary as a function of energy. The energy dependence is conservatively assumed to be of the same order as the absolute scale uncertainties given by the $p_{0}$ terms.

In addition to impacting energy reconstruction, the $\mathrm{E}$ field model also affects the definition of the FD fiducial volume, which is sensitive to electron drift. An additional $1 \%$ uncertainty is assumed on the total fiducial mass, which is conservatively treated as uncorrelated between the $v_{\mu}$ and $v_{e}$ 
samples due to the potential distortion caused by large electromagnetic showers in the electron sample. These uncertainties affect only the overall normalization, and are called FV numu FD and FV nue FD in Fig. 12.

The ND and FD have different acceptance to CC events due to the very different detector sizes. The FD is sufficiently large that acceptance is not expected to vary significantly as a function of event kinematics. However, the ND selection requires that hadronic showers be well contained in LAr to ensure a good energy resolution, resulting in a loss of acceptance for events with energetic hadronic showers. The ND also has regions of muon phase space with lower acceptance due to tracks exiting the side of the TPC but failing to match to the MPD, which are currently not used in the sensitivity analysis.

Uncertainties are evaluated on the muon and hadron acceptance of the ND. The detector acceptance for muons and hadrons is shown in Fig. 5. Inefficiency at very low lepton energy is due to events being misreconstructed as neutral current. For high energy, forward muons, the inefficiency is only due to events near the edge of the FV where the muon happens to miss the MPD. At high transverse momentum, muons begin to exit the side of the LAr active volume, except when they happen to go along the $7 \mathrm{~m}$ axis. The acceptance is sensitive to the modeling of muons in the detector. An uncertainty is estimated based on the change in the acceptance as a function of muon kinematics.

Inefficiency at high hadronic energy is due to the veto on more than $30 \mathrm{MeV}$ deposited in the outer $30 \mathrm{~cm}$ of the LAr active volume. Rejected events are typically poorly reconstructed due to low containment, and the acceptance is expected to decrease at high hadronic energy. Similar to the muon reconstruction, this acceptance is sensitive to detector modeling, and an uncertainty is evaluated based on the change in the acceptance as a function of true hadronic energy.

\section{Sensitivity methods}

Previous DUNE sensitivity predictions have used the GLoBES framework [7,91,92]. In this work, fits are performed using the CAFAna [93] analysis framework, developed originally for the NOvA experiment. Systematics are implemented using one-dimensional response functions for each analysis bin, and oscillation weights are calculated exactly, in fine (50 $\mathrm{MeV}$ ) bins of true neutrino energy. For a given set of inputs, flux, oscillation parameters, cross sections, detector energy response matrices, and detector efficiency, an expected event rate can be produced. Minimization is performed using the MINUIT [94] package.

Oscillation sensitivities are obtained by simultaneously fitting the $v_{\mu} \rightarrow v_{\mu}, \bar{v}_{\mu} \rightarrow \bar{v}_{\mu}$ (Fig. 11), $v_{\mu} \rightarrow v_{e}$, and

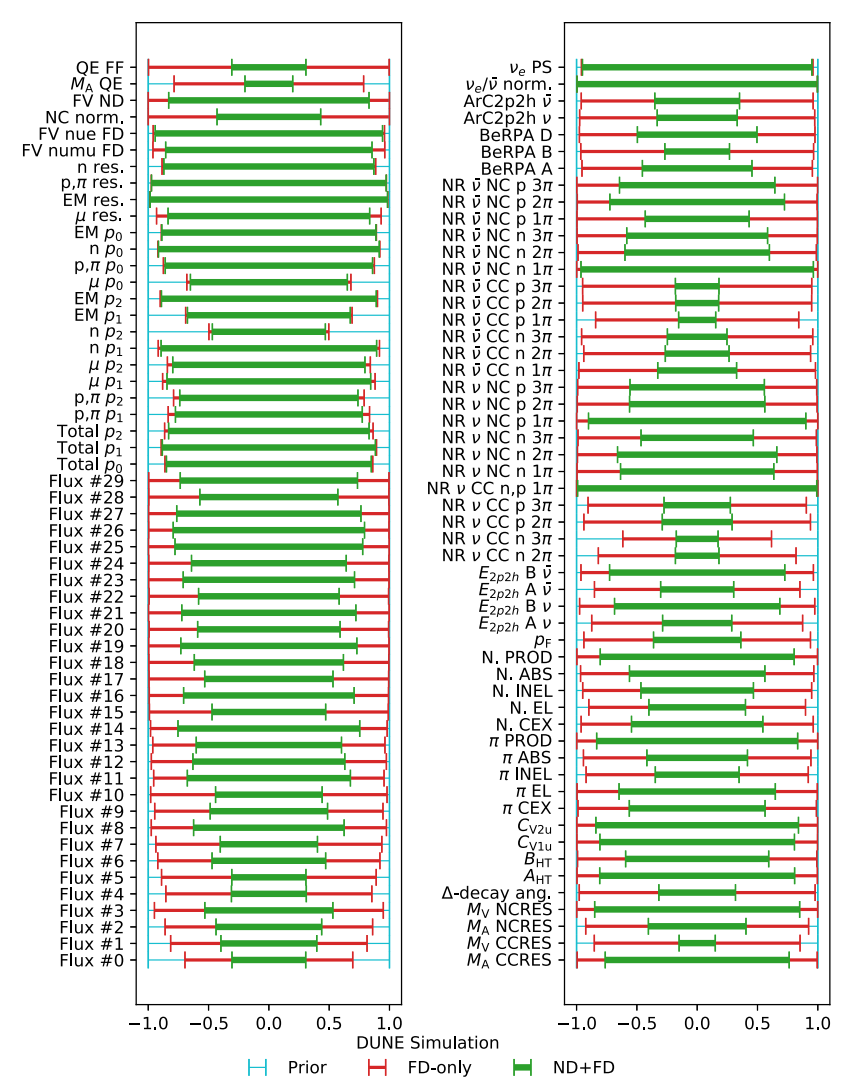

Fig. 12 The ratio of post-fit to pre-fit uncertainties for various systematic parameters for a 15 -year staged exposure. The red band shows the constraint from the FD only in 15 years, while the green shows the ND+FD constraints. Flux parameters are named "Flux \# $i$ " representing the $i$ th principal flux component, cross-section parameter names are given in Sect. 3, and detector systematics are described in Sect. 7, where the $p_{0}, p_{1}$ and $p_{2}$ parameters are described in Table 9

$\bar{v}_{\mu} \rightarrow \bar{v}_{e}$ (Fig. 10) FD spectra along with the $v_{\mu}$ FHC and $\bar{v}_{\mu}$ RHC samples from the ND (Fig. 6). In the studies, all oscillation parameters shown in Table 8 are allowed to vary. Gaussian penalty terms (taken from Table 8 ) are applied to $\theta_{12}$ and $\Delta m_{12}^{2}$ and the matter density, $\rho$, of the Earth along the DUNE baseline [84]. Unless otherwise stated, studies presented here include a Gaussian penalty term on $\theta_{13}$ (also taken from Table 8 ), which is precisely measured by experiments sensitive to reactor antineutrino disappearance [9597]. The remaining parameters, $\sin ^{2} \theta_{23}, \Delta m_{32}^{2}$, and $\delta_{\mathrm{CP}}$ are allowed to vary freely, with no penalty term. Note that the penalty terms are treated as uncorrelated with each other, or other parameters, which is a simplification. In particular, the reactor experiments that drive the constraint on $\theta_{13}$ in the NuFIT analysis are also sensitive to $\Delta m_{32}^{2}$, so the constraint on $\theta_{13}$ should be correlated with $\Delta m_{32}^{2}$. We do not expect this to have a significant impact on the fits, and this effect only matters for those results with the $\theta_{13}$ Gaussian penalty term included. 
Flux, cross section, and FD detector parameters are allowed to vary in the fit, but are constrained by a penalty term proportional to the pre-fit uncertainty. ND detector parameters are not allowed to vary in the fit, but their effect is included via a covariance matrix based on the shape difference between ND prediction and the "data" (which comes from the simulation in this sensitivity study). The covariance matrix is constructed with a throwing technique. For each "throw", all ND energy scale, resolution, and acceptance parameters are simultaneously thrown according to their respective uncertainties, and the modified prediction is produced by varying the relevant quantities away from the nominal prediction according to the thrown parameter values. The bin-to-bin covariance is determined by comparing the resulting spectra with the nominal prediction, in the same binning as is used in the oscillation sensitivity analysis. This choice protects against overconstraining that could occur given the limitations of the parameterized ND reconstruction described in Sect. 4 taken together with the high statistical power at the ND, but is also a simplification.

The compatibility of a particular oscillation hypothesis with both ND and FD data is evaluated using a negative loglikelihood ratio, which converges to a $\chi^{2}$ at high-statistics [48]:

$$
\begin{aligned}
\chi^{2}(\vartheta, \mathbf{x})= & -2 \log \mathcal{L}(\boldsymbol{\vartheta}, \mathbf{x}) \\
= & 2 \sum_{i}^{N_{\text {bins }}}\left[M_{i}(\boldsymbol{\vartheta}, \mathbf{x})-D_{i}+D_{i} \ln \left(\frac{D_{i}}{M_{i}(\vartheta, \mathbf{x})}\right)\right] \\
& +\sum_{j}^{N_{\text {systs }}}\left[\frac{\Delta x_{j}}{\sigma_{j}}\right]^{2} \\
& +\sum_{k}^{N_{\text {bins }}^{\text {ND }}} \sum_{l}^{\text {bD }}\left(M_{k}(\mathbf{x})-D_{k}\right) V_{k l}^{-1}\left(M_{l}(\mathbf{x})-D_{l}\right)
\end{aligned}
$$

where $\vartheta$ and $\mathbf{x}$ are the vector of oscillation parameter and nuisance parameter values respectively; $M_{i}(\boldsymbol{\vartheta}, \mathbf{x})$ and $D_{i}$ are the Monte Carlo (MC) expectation and fake data in the $i$ th reconstructed bin (summed over all selected samples), with the oscillation parameters neglected for the ND; $\Delta x_{j}$ and $\sigma_{j}$ are the difference between the nominal and current value, and the prior uncertainty on the $j$ th nuisance parameter with uncertainties evaluated and described in Sects. 2, 3 and 7; and $V_{k l}$ is the covariance matrix between ND bins described previously. In order to avoid falling into a false minimum, all fits are repeated for four different $\delta_{\mathrm{CP}}$ values $(-\pi,-\pi / 2$, $0, \pi / 2)$, both mass orderings, and in both octants, and the lowest $\chi^{2}$ value is taken as the minimum.

Two approaches are used for the sensitivity studies presented in this work. First, Asimov studies [98] are carried out in which the fake (Asimov) dataset is the same as the nominal MC. In these, the true value of all systematic uncer-
Table 10 Treatment of the oscillation parameters for the simulated data set studies. Note that for some studies $\theta_{13}$ has a Gaussian penalty term applied based on the NuFIT value, and for others it is thrown uniformly within a range determined from the NuFIT $3 \sigma$ allowed range

\begin{tabular}{lll}
\hline Parameter & Prior & Range \\
\hline $\sin ^{2} \theta_{23}$ & Uniform & {$[0.4 ; 0.6]$} \\
$\left|\Delta m_{32}^{2}\right|\left(\times 10^{-3} \mathrm{eV}^{2}\right)$ & Uniform & $|[2.3 ; 2.7]|$ \\
$\delta_{\mathrm{CP}}(\pi)$ & Uniform & {$[-1 ; 1]$} \\
$\theta_{13}$ & Gaussian & NuFIT \\
& Uniform & {$[0.13 ; 0.2]$} \\
\hline
\end{tabular}

tainties and oscillation parameters except those of interest (which are fixed at a test point) remain unchanged, and can vary in the fit, but are constrained by their pre-fit uncertainty. Second, studies are performed where many statistical and systematic throws are made according to their pre-fit Gaussian uncertainties, and fits of all parameters are carried out for each throw. A distribution of post-fit values is built up for the parameter of interest. In these, the expected resolution for oscillation parameters is determined from the spread in best-fit values obtained from an ensemble of throws that vary according to both the statistical and systematic uncertainties. For each throw, the true value of each nuisance parameter is chosen randomly from a distribution determined by the a priori uncertainty on the parameter. For some studies, oscillation parameters are also randomly chosen as described in Table 10. Poisson fluctuations are then applied to all analysis bins, based on the mean event count for each bin after the systematic adjustments have been applied. For each throw in the ensemble, the test statistic is minimized, and the bestfit value of all parameters is determined. The median throw and central $68 \%$ of throws derived from these ensembles are shown.

Sensitivity calculations for CPV, neutrino mass ordering, and octant are performed, in addition to studies of oscillation parameter resolution in one and two dimensions. In these cases, the experimental sensitivity is quantified using a likelihood ratio as the test statistic:

$\Delta \chi^{2}=\chi_{\mathrm{B}}^{2}-\chi_{\mathrm{A}}^{2}$,

where $\chi_{\mathrm{B}}^{2}$ and $\chi_{\mathrm{A}}^{2}$ are both obtained from Eq. (3), using a coherent systematic and statistical throw. The size of $\Delta \chi^{2}$ is a measure of how well the data can exclude model $\mathrm{B}$ in favor of model A, given the uncertainty in the model. For example, the sensitivity for excluding the IO in favor of the NO would be given as $\chi_{\mathrm{IO}}^{2}-\chi_{\mathrm{NO}}^{2}$. Note that the $\Delta \chi^{2}$ for the mass ordering may be negative, depending on how the test is set up. The sensitivity for discovering CPV is the preference for the $\mathrm{CP}$ violating hypothesis over the $\mathrm{CP}$ conserving hypothesis, $\chi_{0, \pi}^{2}-\chi_{\mathrm{CPV}}^{2}$. 
Post-fit uncertainties on systematic parameters are shown for Asimov fits at the NuFIT best-fit point to both the ND+FD samples, and the FD-only samples in Fig. 12, as a fraction of the pre-fit systematic uncertainties described in Sects. 2, 3 , and 7. The FD alone can only weakly constrain the flux and cross-section parameters, which are much more strongly constrained when the ND is included. The ND is, however, unable to strongly constrain the FD detector systematics as they are treated as uncorrelated, and due to the treatment of ND detector systematics in a covariance matrix in Eq. (3). Adding the ND does slightly increase the constraint on detector parameters as it breaks degeneracies with other parameters. Several important cross-section uncertainties are also not constrained by the ND. In particular, an uncertainty on the ratio of $v_{\mu}$ to $v_{e}$ cross sections is totally unconstrained, which is not surprising given the lack of ND $v_{e}$ samples in the current analysis. The most significant flux terms are constrained at the level of $20 \%$ of their a priori values. Less significant principal components have little impact on the observed distributions at either detector, and receive weaker constraints.

Figure 13 shows the pre- and post-fit systematic uncertainties on the FD FHC samples for Asimov fits at the NuFIT best-fit point including both ND and FD samples with a 15 year exposure. It shows how the parameter constraints seen in Fig. 12 translate to a constraint on the event rate. Similar results are seen for the RHC samples. The large reduction in the systematic uncertainties is largely due to the ND constraint on the systematic uncertainties apparent from Fig. 12.

\section{Sensitivities}

In this section, various sensitivity results are presented. For the sake of simplicity, unless otherwise stated, only true normal ordering is shown. Possible variations of sensitivity are presented in two ways. Results produced using Asimovs are shown as lines, and differences between two Asimov scenarios are shown with a colored band. Note that the band in the Asimov case is purely to guide the eye, and does not denote a confidence interval. For results produced using many throws of oscillation parameters, systematic and statistical uncertainties, $\sim 300,000$ throws were used to calculate the sensitivity for each scenario. The median sensitivity is shown with a solid line, and a transparent filled area indicates the region containing the central $68 \%$ of throws, which can be interpreted as the $1 \sigma$ uncertainty on the sensitivity.

Figure 14 shows the significance with which $\mathrm{CPV}\left(\delta_{\mathrm{CP}} \neq\right.$ $[0, \pm \pi])$ can be observed in both NO and IO as a function of the true value of $\delta_{\mathrm{CP}}$ for exposures corresponding to seven and ten years of data, using the staging scenario described in Sect. 6, and using the toy throwing method described in Sect. 8 to investigate their effect on the sensitivity. This sensi-
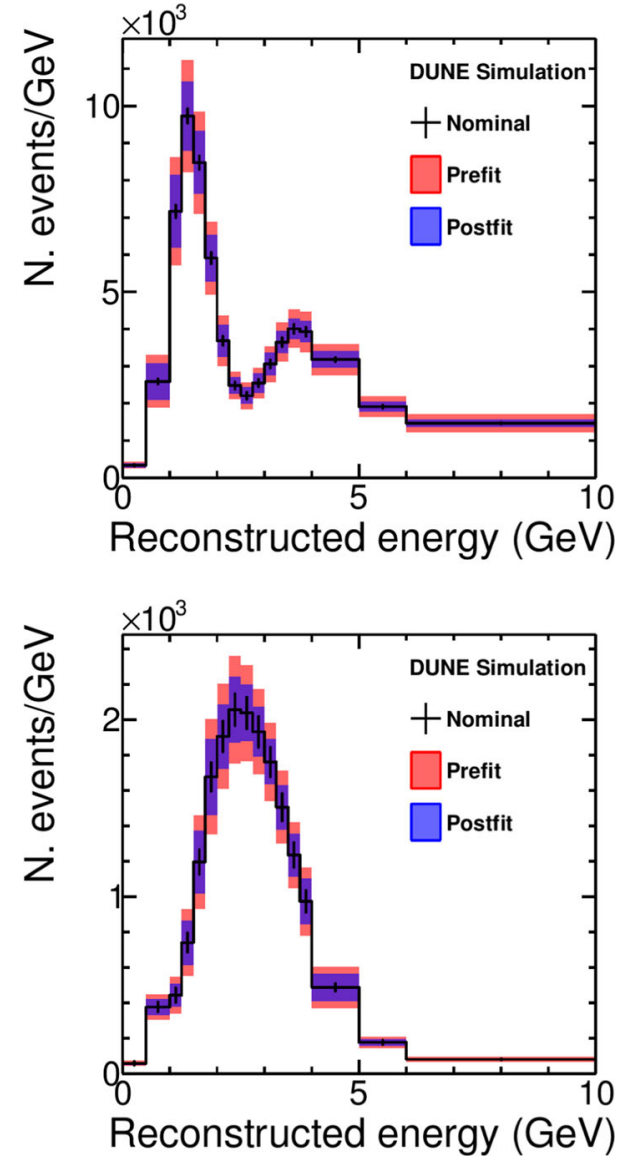

Fig. $13 v_{\mu}$ (top) and $v_{e}$ (bottom) FD FHC spectra for a 15 year staged exposure with oscillation parameters set to the NuFIT best-fit point, shown as a function of reconstructed neutrino energy. The statistical uncertainty on the total rate is shown on the data points, and the preand post-fit systematic uncertainties are shown as shaded bands. The post-fit uncertainty includes the effect of the ND samples in the fit, and corresponds to the parameter constraints shown in Fig. 12

tivity has a characteristic double peak structure because the significance of a CPV measurement necessarily decreases around CP-conserving values of $\delta_{\mathrm{CP}}$. The median CPV sensitivity reaches $5 \sigma$ for a small range of values after an exposure of seven years in NO, and a broad range of values after a ten year exposure. In IO, DUNE has slightly stronger sensitivity to $\mathrm{CPV}$, and reaches $5 \sigma$ for a broad range of values after a seven year exposure. Note that with statistical and systematic throws, the median sensitivity never reaches exactly zero.

Figure 15 shows the DUNE Asimov sensitivity to CPV in NO when the true values of $\theta_{23}, \theta_{13}$, and $\Delta m_{32}^{2}$ vary within the $3 \sigma$ range allowed by NuFIT. The largest effect is the variation in sensitivity with the true value of $\theta_{23}$, where degeneracy with $\delta_{\mathrm{CP}}$ and matter effects are significant. Values of $\theta_{23}$ in the lower octant lead to the best sensitivity to CPV. The true values of $\theta_{13}$ and $\Delta m_{32}^{2}$ are highly constrained by global data and, within these constraints, do not have a dramatic impact on the sensitivity. Note that in the Asimov cases shown in 

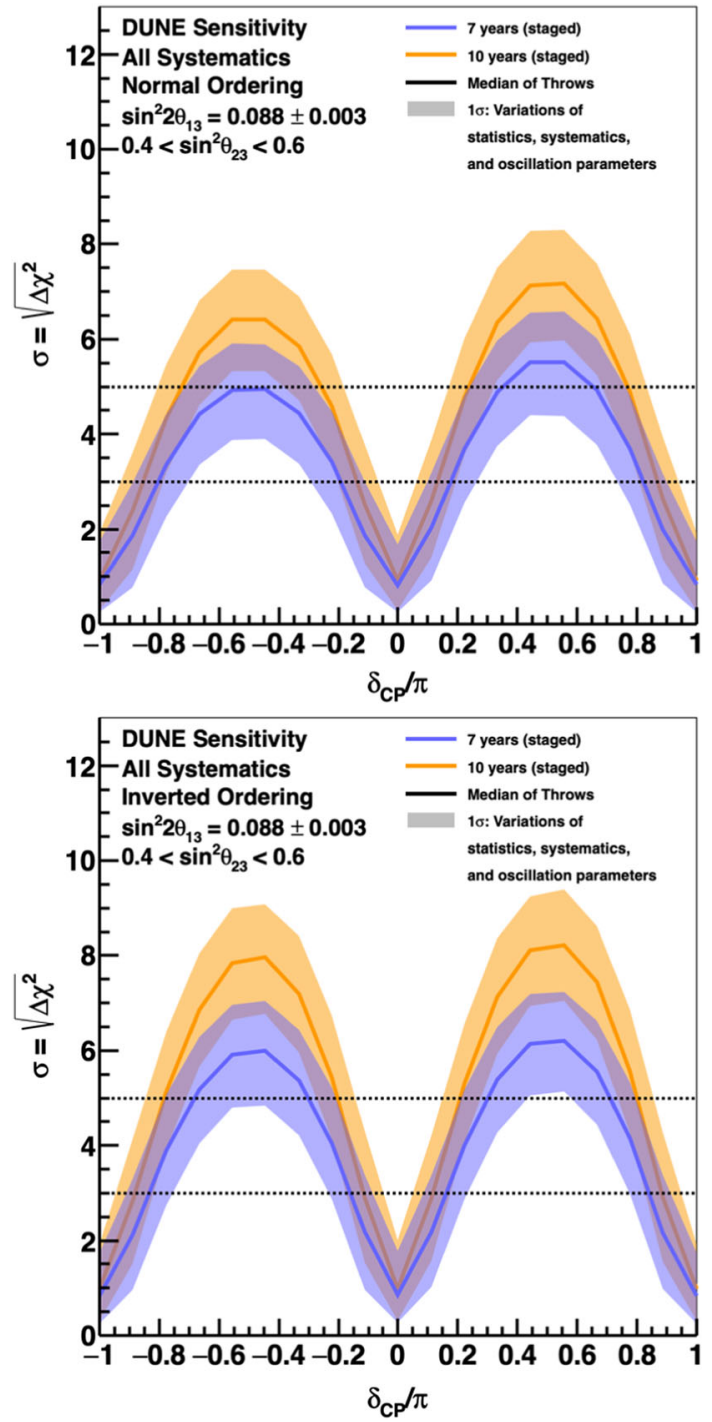

Fig. 14 Significance of the DUNE determination of CP-violation $\left(\delta_{\mathrm{CP}} \neq[0, \pm \pi]\right)$ as a function of the true value of $\delta_{\mathrm{CP}}$, for seven (blue) and ten (orange) years of exposure, in both normal (top) and inverted (bottom) ordering. The width of the transparent bands cover $68 \%$ of fits in which random throws are used to simulate statistical variations and select true values of the oscillation and systematic uncertainty parameters, constrained by pre-fit uncertainties. The solid lines show the median sensitivity

Fig. 15, the median sensitivity reaches 0 at $\mathrm{CP}$-conserving values of $\delta_{\mathrm{CP}}$ (unlike the case with the throws as in Fig. 14), but in regions far from $\mathrm{CP}$-conserving values, the Asimov sensitivity and the median sensitivity from the throws agree well.

Figure 16 shows the result of Asimov studies investigating the significance with which CPV can be determined in NO for $75 \%$ and $50 \%$ of $\delta_{\mathrm{CP}}$ values, and when $\delta_{\mathrm{CP}}=-\pi / 2$, as a function of exposure in kt-MW-years, which can be converted to years using the staging scenario described in Sect. 6. The width of the bands show the impact of applying an exter-
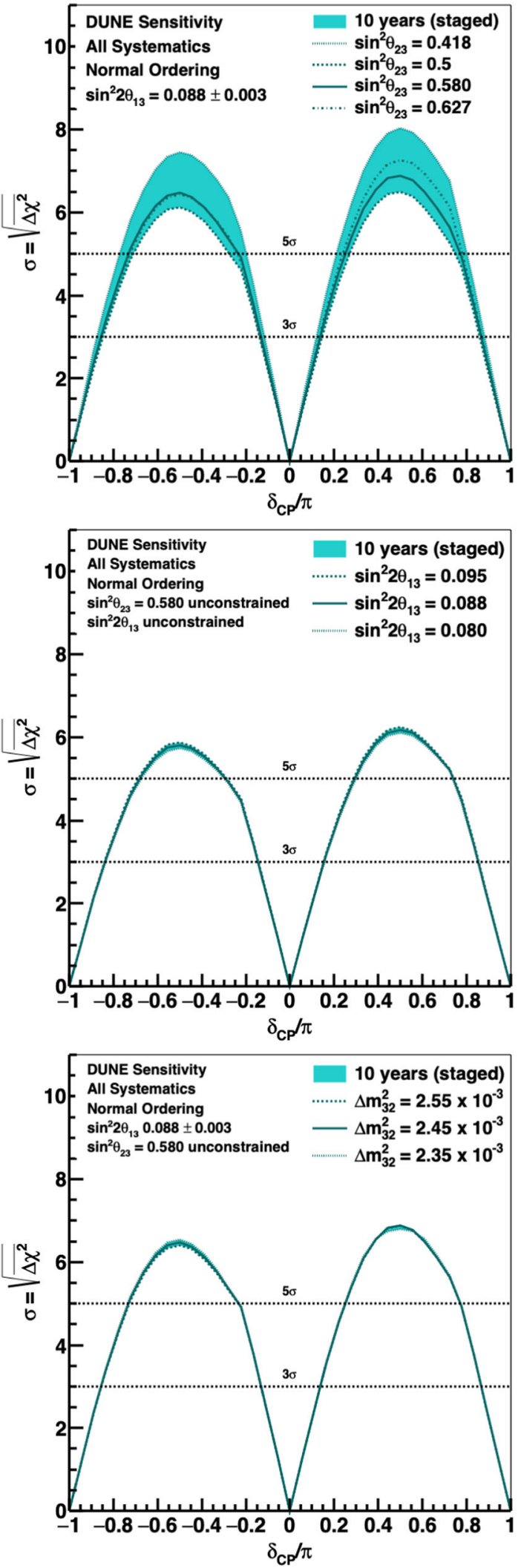

Fig. 15 Asimov sensitivity to $\mathrm{CP}$ violation, as a function of the true value of $\delta_{\mathrm{CP}}$, for ten years of exposure. Curves are shown for variations in the true values of $\theta_{23}$ (top), $\theta_{13}$ (middle) and $\Delta m_{32}^{2}$ (bottom), which correspond to their $3 \sigma$ NuFIT range of values, as well as the NuFIT central value, and maximal mixing 

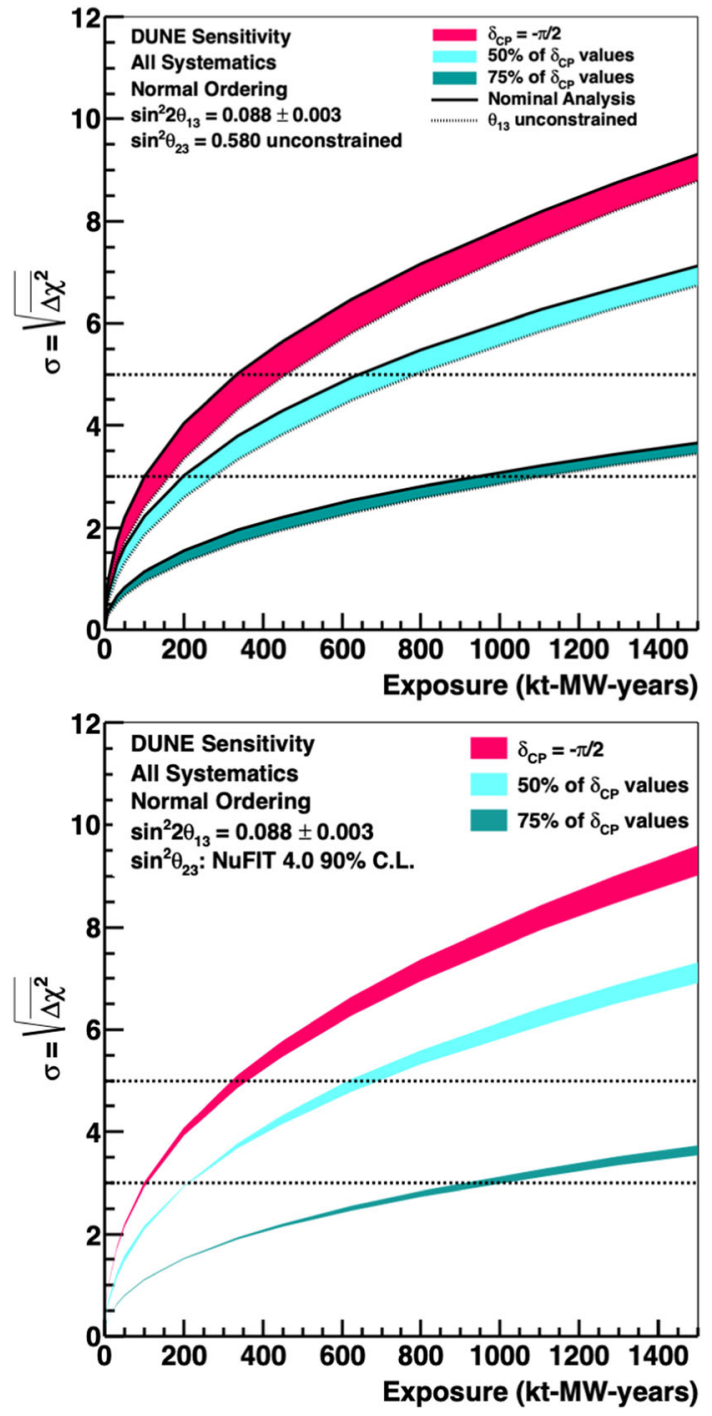

Fig. 16 Significance of the DUNE determination of CP-violation $\left(\delta_{\mathrm{CP}} \neq[0, \pi]\right)$ for the case when $\delta_{\mathrm{CP}}=-\pi / 2$, and for $50 \%$ and $75 \%$ of possible true $\delta_{\mathrm{CP}}$ values, as a function of exposure in kt-MW-years. Top: The width of the band shows the impact of applying an external constraint on $\theta_{13}$. Bottom: The width of the band shows the impact of varying the true value of $\sin ^{2} \theta_{23}$ within the NuFIT $90 \%$ C.L. region

nal constraint on $\theta_{13}$. CP violation can be observed with $5 \sigma$ significance after about seven years (336 kt-MW-years) if $\delta_{\mathrm{CP}}=-\pi / 2$ and after about ten years (624 kt-MW-years) for $50 \%$ of $\delta_{\mathrm{CP}}$ values. CP violation can be observed with $3 \sigma$ significance for $75 \%$ of $\delta_{\mathrm{CP}}$ values after about 13 years of running. In the bottom plot of Fig. 16, the width of the bands shows the impact of applying an external constraint on $\theta_{13}$, while in the bottom plot, the width of the bands is the result of varying the true value of $\sin ^{2} \theta_{23}$ within the NuFIT $90 \%$ C.L. allowed region.

Figure 17 shows the significance with which the neutrino mass ordering can be determined in both NO and IO as a function of the true value of $\delta_{\mathrm{CP}}$, for both seven and ten
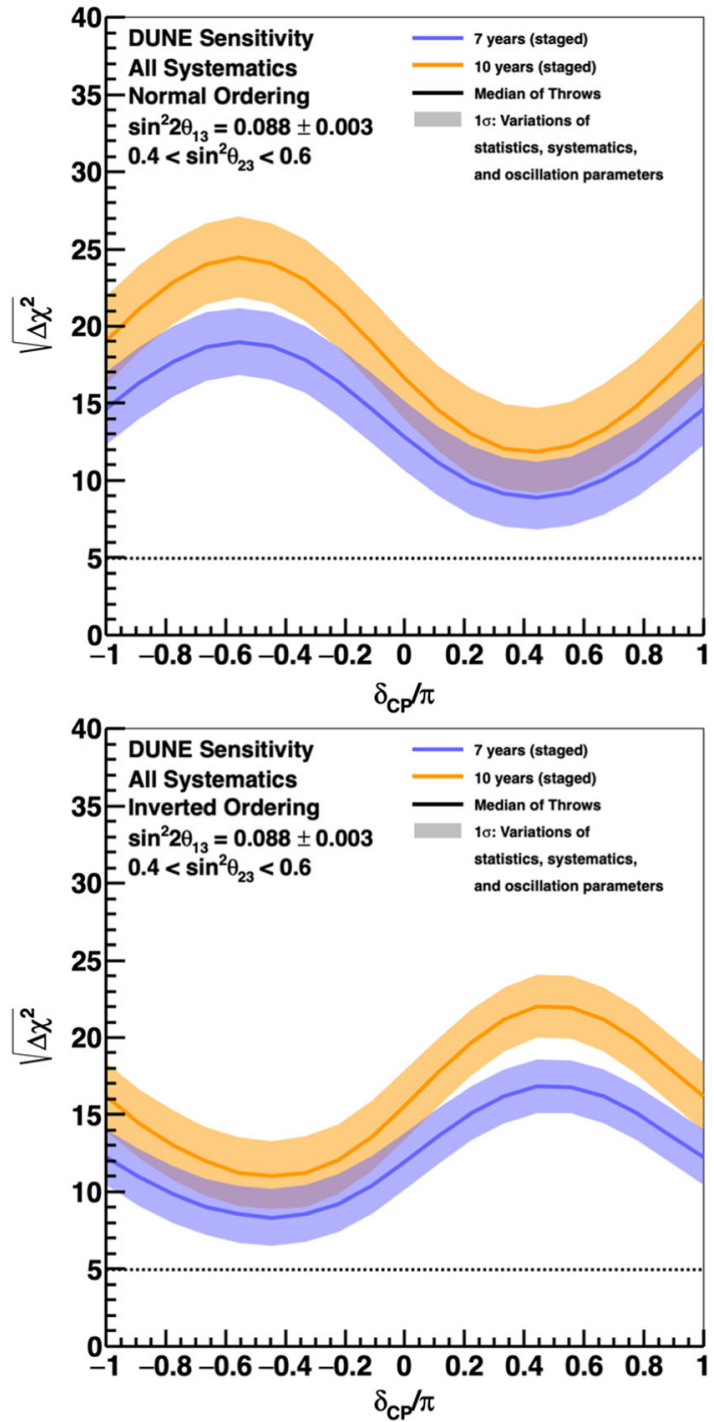

Fig. 17 Significance of the DUNE determination of the neutrino mass ordering, as a function of the true value of $\delta_{\mathrm{CP}}$, for seven (blue) and ten (orange) years of exposure. The width of the transparent bands cover $68 \%$ of fits in which random throws are used to simulate statistical variations and select true values of the oscillation and systematic uncertainty parameters, constrained by pre-fit uncertainties. The solid lines show the median sensitivity

year exposures, including the effect of all other oscillation and systematic parameters using the toy throwing method described in Sect. 8. The characteristic shape results from near degeneracy between matter and CPV effects that occurs near $\delta_{\mathrm{CP}}=\pi / 2\left(-\delta_{\mathrm{CP}}=\pi / 2\right)$ for true normal (inverted) ordering. Studies have indicated that special attention must be paid to the statistical interpretation of neutrino mass ordering sensitivities [99-101] because the $\Delta \chi^{2}$ metric does not follow the expected chi-square function for one degree of freedom, so the interpretation of the $\sqrt{\Delta \chi^{2}}$ as the sensitivity is complicated. However, it is clear from Fig. 17 that DUNE is able to distinguish the mass ordering for both true $\mathrm{NO}$ and 


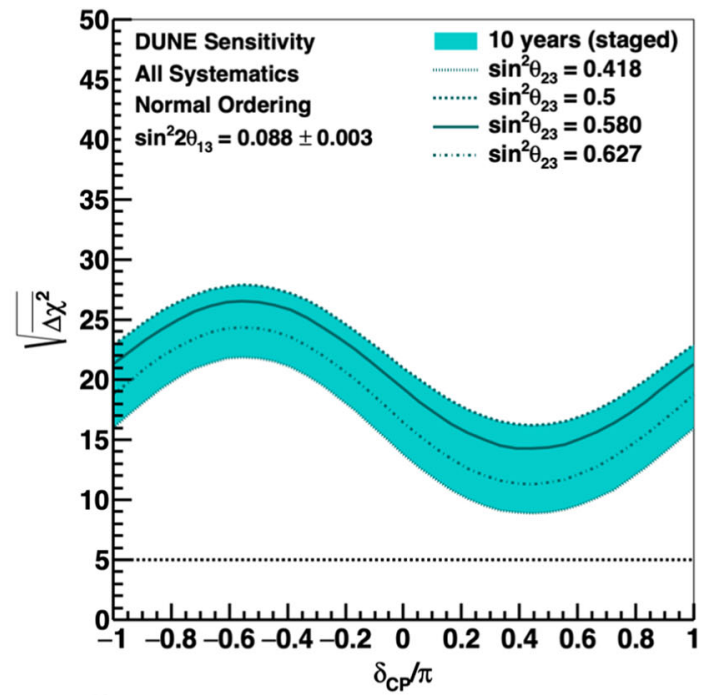

IO, and using the corrections from, for example, Ref. [99], DUNE would still achieve $5 \sigma$ significance for the central $68 \%$ of all throws shown in Fig. 17. We note that for both seven and ten years (it was not checked for lower exposures), there were no parameter throws used in generating the plots ( $\sim 300,000$ each) for which the incorrect mass ordering was preferred.

Figure 18 shows the DUNE Asimov sensitivity to the neutrino mass ordering when the true values of $\theta_{23}, \theta_{13}$, and $\Delta m_{32}^{2}$ vary within the $3 \sigma$ range allowed by NuFIT. As for CPV (in Fig. 15), the largest variation in sensitivity is with the true value of $\theta_{23}$, but in this case, the upper octant leads to the best sensitivity. Again, the true values of $\theta_{13}$ and $\Delta m_{32}^{2}$ do not have a dramatic impact on the sensitivity. The median
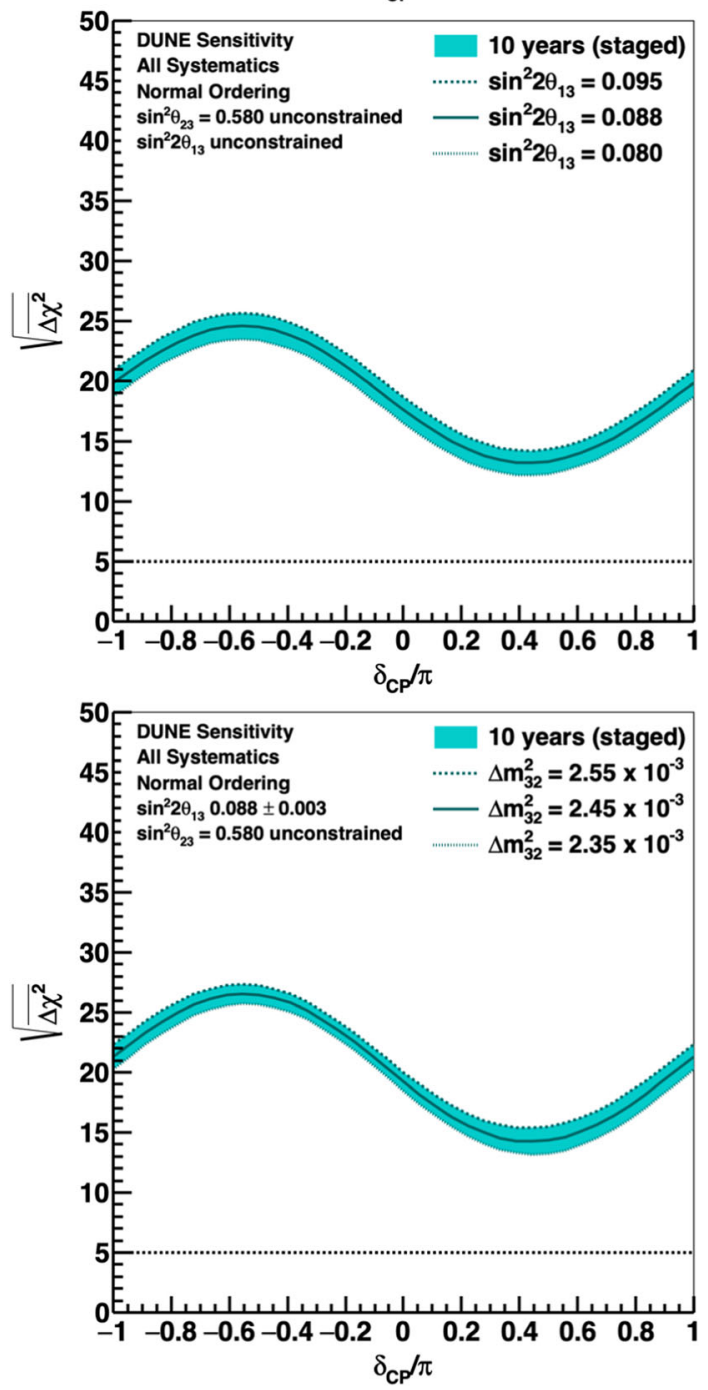

Fig. 18 Asimov sensitivity to the neutrino mass ordering, as a function of the true value of $\delta_{\mathrm{CP}}$, for ten years of exposure. Curves are shown for variations in the true values of $\theta_{23}$ (top), $\theta_{13}$ (middle) and $\Delta m_{32}^{2}$ (bottom), which correspond to their $3 \sigma$ NuFIT range of values, as well as the NuFIT central value. and maximal mixing
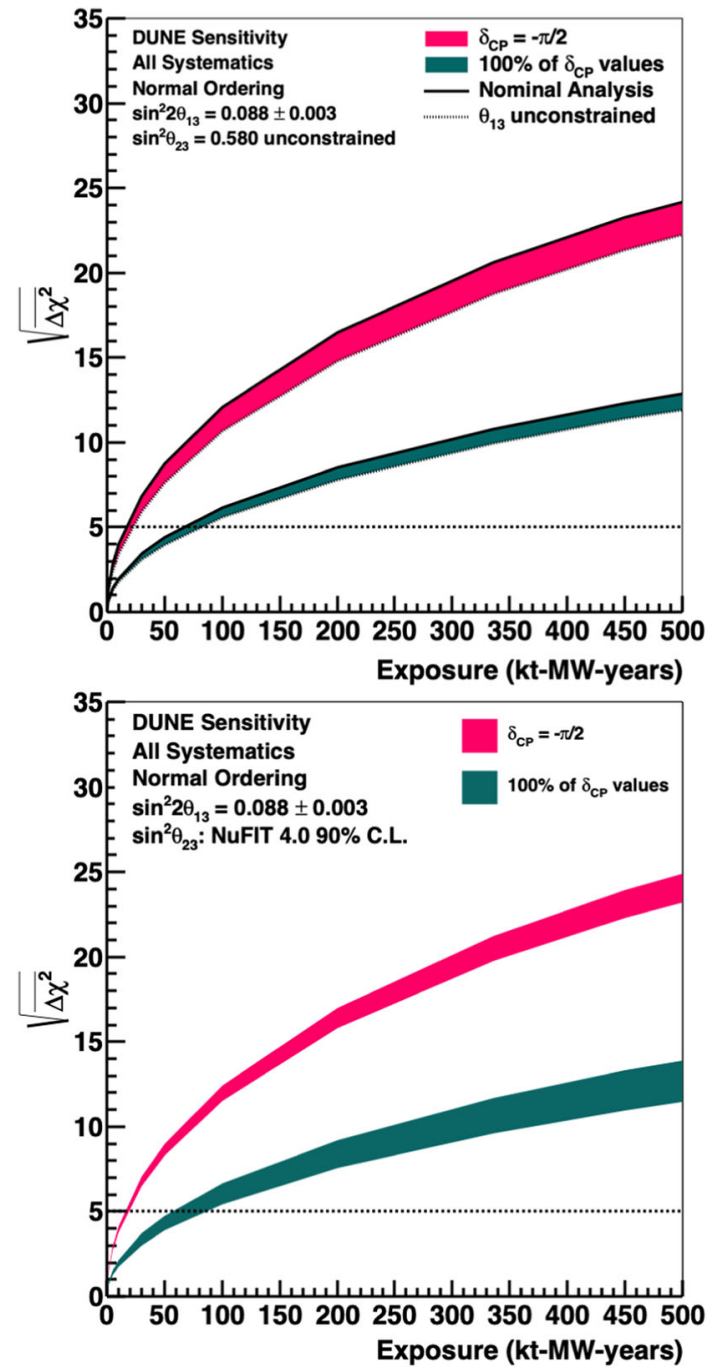

Fig. 19 Significance of the DUNE determination of the neutrino mass ordering for the case when $\delta_{\mathrm{CP}}=-\pi / 2$, and for $100 \%$ of possible true $\delta_{\mathrm{CP}}$ values, as a function of exposure in kt-MW-years. Top: The width of the band shows the impact of applying an external constraint on $\theta_{13}$. Bottom: The width of the band shows the impact of varying the true value of $\sin ^{2} \theta_{23}$ within the NuFIT 90\% C.L. region 

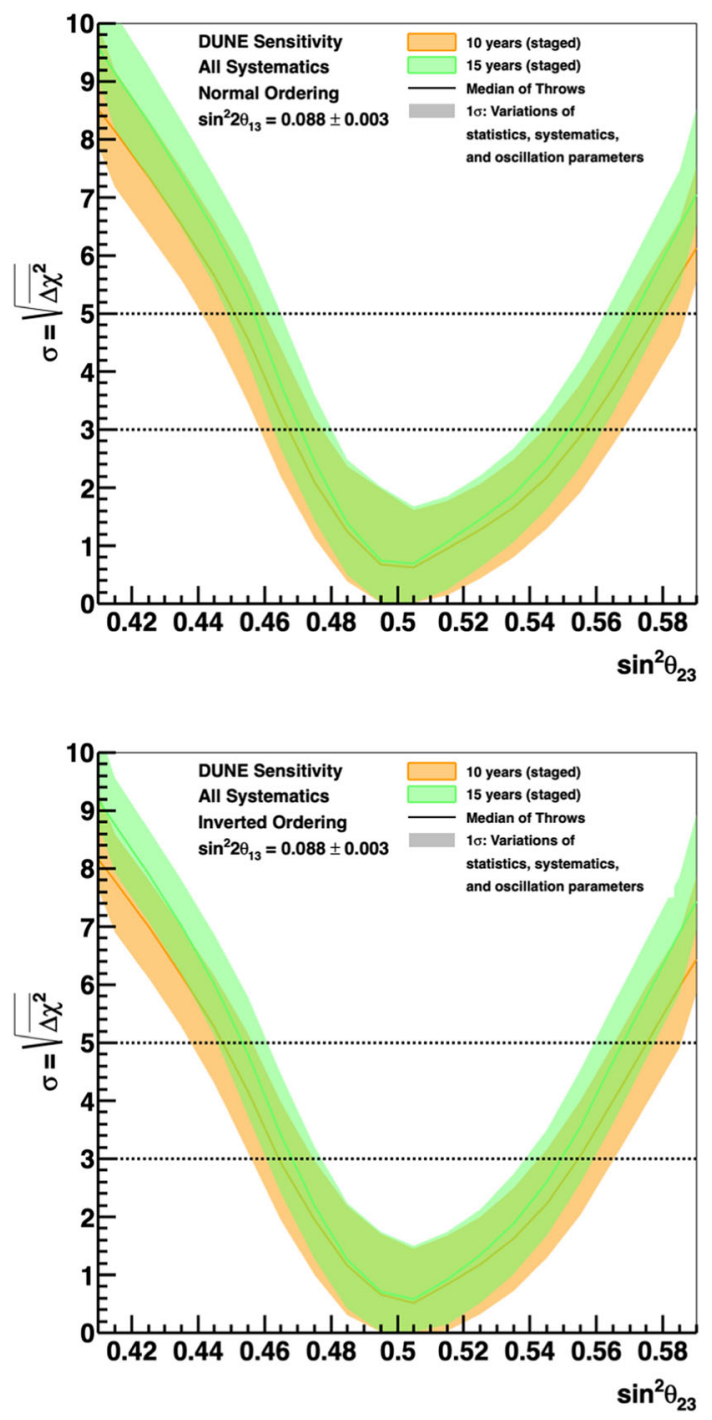

Fig. 20 Sensitivity to determination of the $\theta_{23}$ octant as a function of the true value of $\sin ^{2} \theta_{23}$, for ten (orange) and fifteen (green) years of exposure, for both normal (top) and inverted (bottom) ordering. The width of the transparent bands cover $68 \%$ of fits in which random throws are used to simulate statistical variations and select true values of the oscillation and systematic uncertainty parameters, constrained by pre-fit uncertainties. The solid lines show the median sensitivity

Asimov sensitivity tracks the median throws shown in Fig. 17 well for the reasonably high exposures tested - this was not checked for exposures below seven years (336 kt-MW-years).

Figure 19 shows the result of Asimov studies assessing the significance with which the neutrino mass ordering can be determined for $100 \%$ of $\delta_{\mathrm{CP}}$ values, and when $\delta_{\mathrm{CP}}=$ $-\pi / 2$, as a function of exposure in kt-MW-years, for true NO. The width of the bands show the impact of applying an external constraint on $\theta_{13}$. The bottom plot shows the impact of varying the true value of $\sin ^{2} \theta_{23}$ within the NuFIT $90 \%$ C.L. region. As DUNE will be able to establish the neutrino mass ordering at the $5 \sigma$ level for $100 \%$ of $\delta_{\mathrm{CP}}$ values after

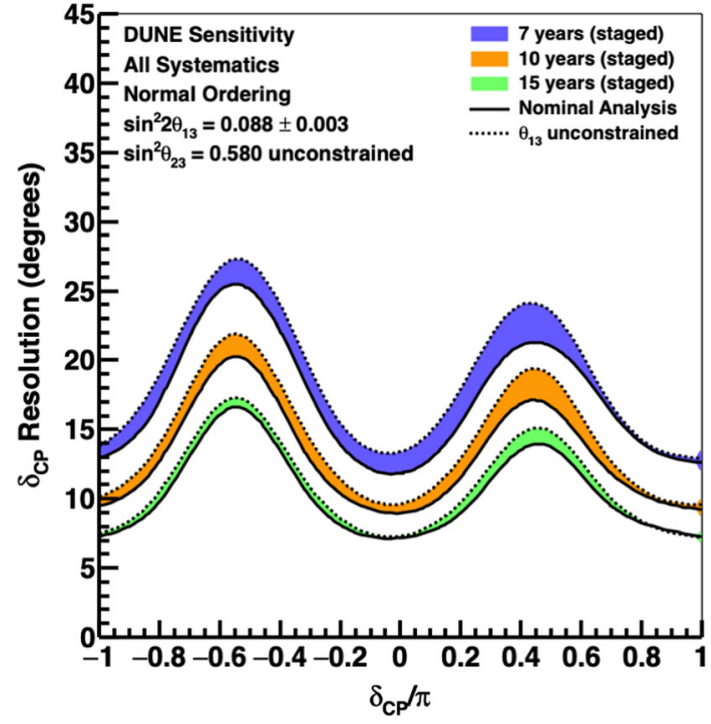

Fig. 21 Resolution in degrees for the DUNE measurement of $\delta_{\mathrm{CP}}$, as a function of the true value of $\delta_{\mathrm{CP}}$, for seven (blue), ten (orange), and fifteen (green) years of exposure. The width of the band shows the impact of applying an external constraint on $\theta_{13}$

a relatively short period, these plots only extend to $500 \mathrm{kt}-$ MW-years.

The measurement of $v_{\mu} \rightarrow v_{\mu}$ oscillations depends on $\sin ^{2} 2 \theta_{23}$, whereas the measurement of $v_{\mu} \rightarrow v_{e}$ oscillations depends on $\sin ^{2} \theta_{23}$. A combination of both $v_{e}$ appearance and $v_{\mu}$ disappearance measurements can probe both maximal mixing and the $\theta_{23}$ octant. Figure 20 shows the sensitivity to determining the octant as a function of the true value of $\sin ^{2} \theta_{23}$, in both NO and IO. We note that the octant sensitivity strongly depends on the use of the external $\theta_{13}$ constraint.

In addition to the discovery potential for neutrino the mass ordering and CPV, and sensitivity to the $\theta_{23}$ octant, DUNE will improve the precision on key parameters that govern neutrino oscillations, including $\delta_{\mathrm{CP}}, \sin ^{2} 2 \theta_{13}, \Delta m_{31}^{2}$, and $\sin ^{2} \theta_{23}$.

Figure 21 shows the resolution, in degrees, of DUNE's measurement of $\delta_{\mathrm{CP}}$, as a function of the true value of $\delta_{\mathrm{CP}}$, for true NO. The resolution on a parameter is produced from the central $68 \%$ of post-fit parameter values using many throws of the systematic and remaining oscillation parameters, and statistical throws. The resolution of this measurement is significantly better near $\mathrm{CP}$-conserving values of $\delta_{\mathrm{CP}}$, compared to maximally $\mathrm{CP}$-violating values. For fifteen years of exposure, resolutions between $5^{\circ}-15^{\circ}$ are possible, depending on the true value of $\delta_{\mathrm{CP}}$. A smoothing algorithm has been applied to interpolate between values of $\delta_{\mathrm{CP}}$ at which the full analysis has been performed.

Figures 22 and 23 show the resolution of DUNE's measurements of $\delta_{\mathrm{CP}}$ and $\sin ^{2} 2 \theta_{13}$ and of $\sin ^{2} 2 \theta_{23}$ and $\Delta m_{32}^{2}$, respectively, as a function of exposure in kt-MW-years. The 

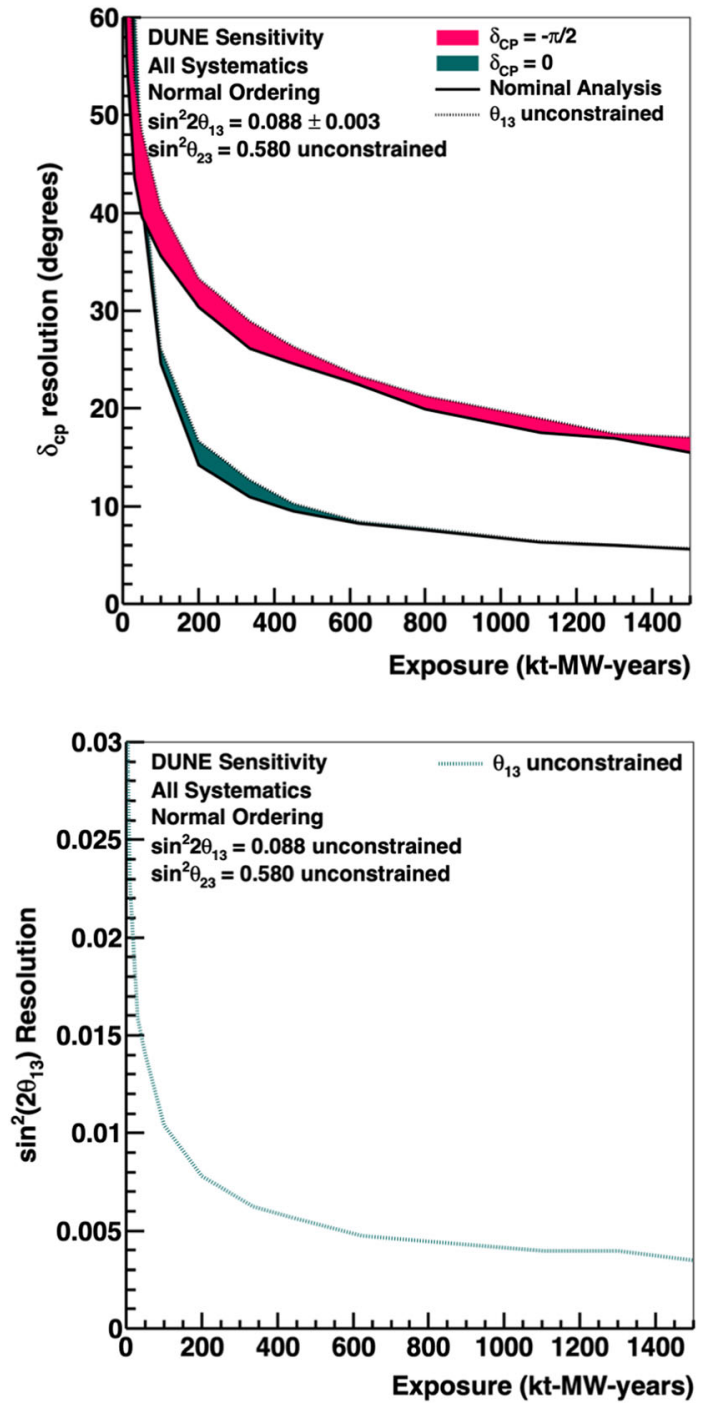

Fig. 22 Resolution of DUNE measurements of $\delta_{\mathrm{CP}}$ (top) and $\sin ^{2} 2 \theta_{13}$ (bottom), as a function of exposure in kt-MW-years. As seen in Fig. 21, the $\delta_{\mathrm{CP}}$ resolution has a significant dependence on the true value of $\delta_{\mathrm{CP}}$, so curves for $\delta_{\mathrm{CP}}=-\pi / 2$ (red) and $\delta_{\mathrm{CP}}=0$ (green) are shown. For $\delta_{\mathrm{CP}}$, the width of the band shows the impact of applying an external constraint on $\theta_{13}$. No constraint is applied when calculating the $\sin ^{2} 2 \theta_{13}$ resolution

resolution on a parameter is produced from the central $68 \%$ of post-fit parameter values using many throws of the systematic other oscillation parameters, and statistical throws. As seen in Fig. 21, the $\delta_{\mathrm{CP}}$ resolution varies significantly with the true value of $\delta_{\mathrm{CP}}$, but for favorable values, resolutions near $5^{\circ}$ are possible for large exposure. The DUNE measurement of $\sin ^{2} 2 \theta_{13}$ approaches the precision of reactor experiments for high exposure, allowing a comparison between the two results, which is of interest as a test of the unitarity of the PMNS matrix.
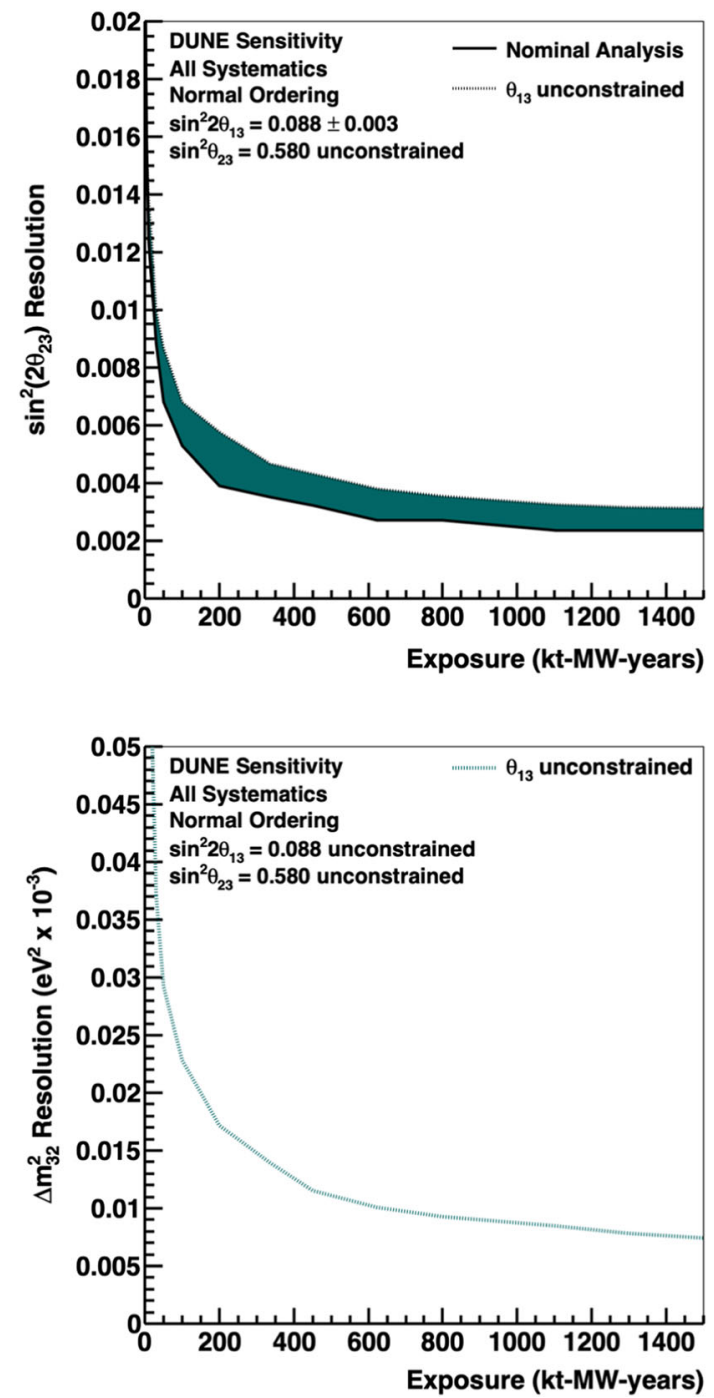

Fig. 23 Resolution of DUNE measurements of $\sin ^{2} 2 \theta_{23}$ (top) and $\Delta m_{32}^{2}$ (bottom), as a function of exposure in kt-MW-years. The width of the band for the $\sin ^{2} 2 \theta_{23}$ resolution shows the impact of applying an external constraint on $\theta_{13}$. For the $\Delta m_{32}^{2}$ resolution, an external constraint does not have a significant impact, so only the unconstrained curve is shown

One of the primary physics goals for DUNE is the simultaneous measurement of all oscillation parameters governing long-baseline neutrino oscillation, without a need for external constraints. Figure 24 shows the $90 \%$ constant $\Delta \chi^{2}$ allowed regions in the $\sin ^{2} 2 \theta_{13}-\delta_{\mathrm{CP}}$ and $\sin ^{2} \theta_{23}-\Delta m_{32}^{2}$ planes for seven, ten, and fifteen years of running, when no external constraints are applied, compared to the current measurements from world data. An additional degenerate lobe visible at higher values of $\sin ^{2} 2 \theta_{13}$ and in the wrong $\sin ^{2} \theta_{23}$ octant is present in the seven and ten year exposures, but is resolved 

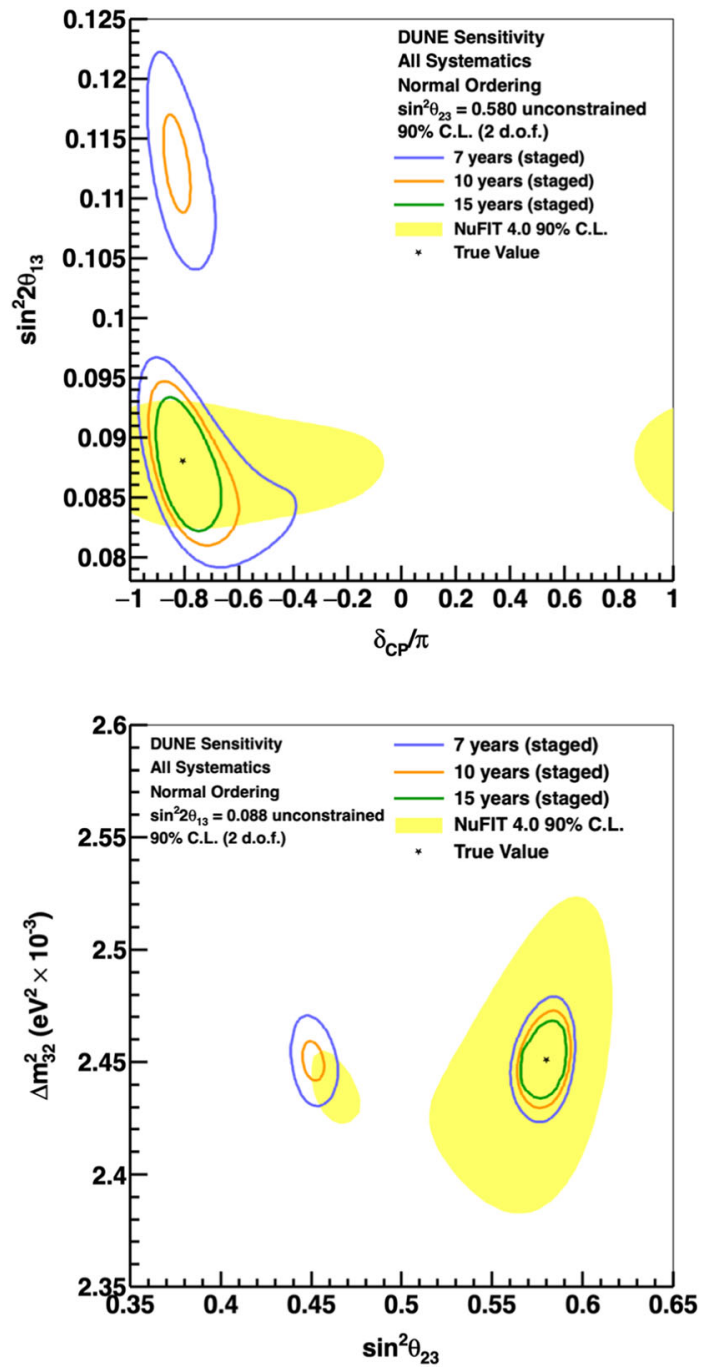

Fig. 24 Two-dimensional $90 \%$ constant $\Delta \chi^{2}$ confidence regions in the $\sin ^{2} 2 \theta_{13}-\delta_{\mathrm{CP}}$ (top) and $\sin ^{2} \theta_{23}-\Delta m_{32}^{2}$ (botton) planes, for seven, ten, and fifteen years of exposure, with equal running in neutrino and antineutrino mode. The $90 \%$ C.L. region for the NuFIT global fit is shown in yellow for comparison. The true values of the oscillation parameters are assumed to be the central values of the NuFIT global fit and the oscillation parameters governing long-baseline oscillation are unconstrained

after long exposures. The time to resolve the degeneracy with DUNE data alone depends on the true oscillation parameter values. For shorter exposures, the degeneracy observed in Fig. 24 can be resolved by introducing an external constraint on the value of $\theta_{13}$. Figure 25 shows two-dimensional $90 \%$ constant $\Delta \chi^{2}$ allowed regions in the $\sin ^{2} \theta_{23}-\delta_{\mathrm{CP}}$ plane with an external constraint on $\theta_{13}$ applied. In this case, the degenerate octant solution has disappeared for all exposures shown.

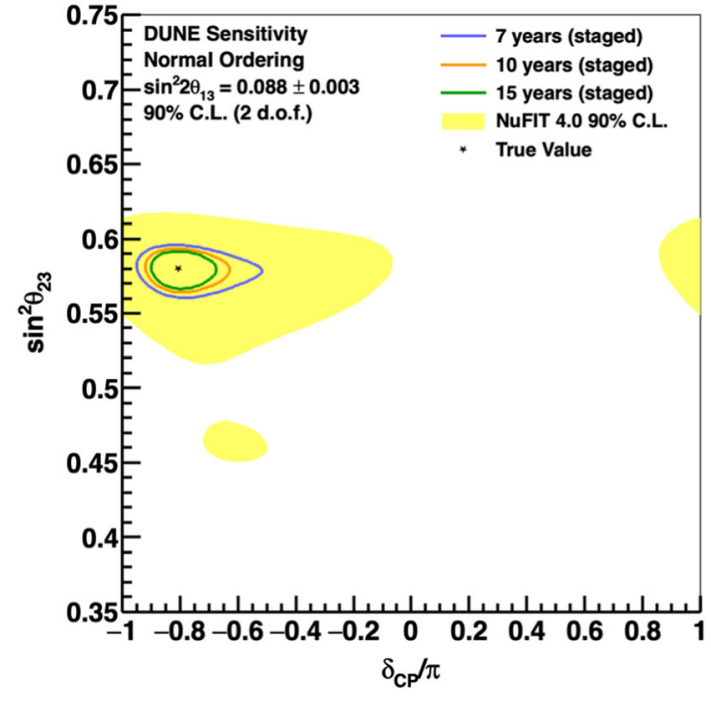

Fig. 25 Two-dimensional $90 \%$ constant $\Delta \chi^{2}$ confidence regions in $\sin ^{2} \theta_{23}-\delta_{\mathrm{CP}}$ plane, for seven, ten, and fifteen years of exposure, with equal running in neutrino and antineutrino mode. The $90 \%$ C.L. region for the NuFIT global fit is shown in yellow for comparison. The true values of the oscillation parameters are assumed to be the central values of the NuFIT global fit and $\theta_{13}$ is constrained by NuFIT

Figure 26 explores the resolution sensitivity that is expected in the $\sin ^{2} \theta_{23}-\delta_{\mathrm{CP}}$ and $\sin ^{2} \theta_{23}-\Delta m_{32}^{2}$ planes for various true oscillation parameter values, with an external constraint on $\theta_{13}$. The true oscillation parameter values used are denoted by stars, and the NuFIT best fit values are used as the true value of all those not explicitly shown. Values of $\sin ^{2} \theta_{23}=0.42,0.5,0.58$ were used in both planes, and additionally, values of $\delta_{\mathrm{CP}}=-\pi / 2,0, \pi / 2$ were used in the $\sin ^{2} \theta_{23}-\delta_{\mathrm{CP}}$ plane. It can be observed that the resolution in the value of $\sin ^{2} \theta_{23}$ is worse at $\sin ^{2} \theta_{23}=0.5$, and improves for values away from maximal in either octant. As was seen in Fig. 21, the resolution of $\delta_{\mathrm{CP}}$ is smaller near the $\mathrm{CP}$-conserving value of $\delta_{\mathrm{CP}}=0$, and increases towards the maximally CP-violating values $\delta_{\mathrm{CP}}= \pm \pi / 2$.

The exposures required to reach selected sensitivity milestones for the nominal analysis are summarized in Table 11. Note that the sensitivity to CPV and for determining the neutrino mass ordering was shown to be dependent on the value of $\theta_{23}$ in Figs. 15 and 18, so these milestones should be treated as approximate. $\delta_{\mathrm{CP}}=-\pi / 2$ is taken as a reference value of maximal CPV close to the current global best fit. Similarly, a resolution of 0.004 on $\sin ^{2} 2 \theta_{13}$ is used as a reference as the current resolution obtained by reactor experiments. 


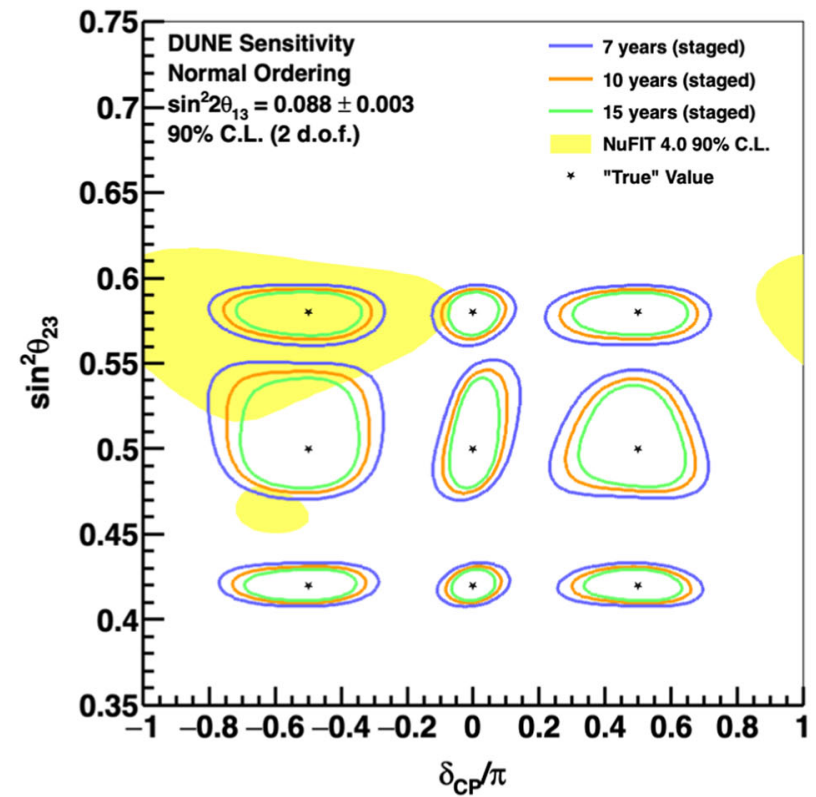

Fig. 26 Two-dimensional $90 \%$ constant $\Delta \chi^{2}$ confidence regions in the $\sin ^{2} \theta_{23}-\delta_{\mathrm{CP}}$ (left) and $\sin ^{2} \theta_{23}-\Delta m_{32}^{2}$ (right) planes for different oscillation parameter values and seven, ten, and fifteen years of exposure, with equal running in neutrino and antineutrino mode. The $90 \%$ C.L. region for the NuFIT global fit is included in yellow for comparison. In all cases, an external constraint on the value of $\theta_{13}$ is applied. The true

Table 11 Exposure in years, assuming true normal ordering and equal running in neutrino and antineutrino mode, required to reach selected physics milestones in the nominal analysis, using the NuFIT best-fit values for the oscillation parameters. The staging scenario described in Sect. 6 is assumed. Exposures are rounded to the nearest year

\begin{tabular}{|c|c|c|}
\hline $\begin{array}{l}\text { Physics milestone } \\
\left(\sin ^{2} \theta_{23}=0.580\right)\end{array}$ & $\begin{array}{l}\text { Exposure } \\
\text { Staged years }\end{array}$ & kt-MW-years \\
\hline $\begin{array}{l}5 \sigma \text { mass ordering } \\
\delta_{\mathrm{CP}}=-\pi / 2\end{array}$ & 1 & 16 \\
\hline $\begin{array}{l}5 \sigma \text { mass ordering } \\
100 \% \text { of } \delta_{\mathrm{CP}} \text { values }\end{array}$ & 2 & 66 \\
\hline $\begin{array}{l}3 \sigma \text { CP violation } \\
\delta_{\mathrm{CP}}=-\pi / 2\end{array}$ & 3 & 100 \\
\hline $\begin{array}{l}3 \sigma \mathrm{CP} \text { violation } \\
50 \% \text { of } \delta_{\mathrm{CP}} \text { values }\end{array}$ & 5 & 197 \\
\hline $\begin{array}{l}5 \sigma \mathrm{CP} \text { violation } \\
\delta_{\mathrm{CP}}=-\pi / 2\end{array}$ & 7 & 334 \\
\hline $\begin{array}{l}5 \sigma \mathrm{CP} \text { violation } \\
50 \% \text { of } \delta_{\mathrm{CP}} \text { values }\end{array}$ & 10 & 646 \\
\hline $\begin{array}{l}3 \sigma \mathrm{CP} \text { violation } \\
75 \% \text { of } \delta_{\mathrm{CP}} \text { values }\end{array}$ & 13 & 936 \\
\hline $\begin{array}{l}\delta_{\mathrm{CP}} \text { resolution of } 10^{\circ} \\
\delta_{\mathrm{CP}}=0\end{array}$ & 8 & 400 \\
\hline $\begin{array}{l}\delta_{\mathrm{CP}} \text { resolution of } 20^{\circ} \\
\delta_{\mathrm{CP}}=-\pi / 2\end{array}$ & 12 & 806 \\
\hline $\sin ^{2} 2 \theta_{13}$ resolution of 0.004 & 15 & 1079 \\
\hline
\end{tabular}

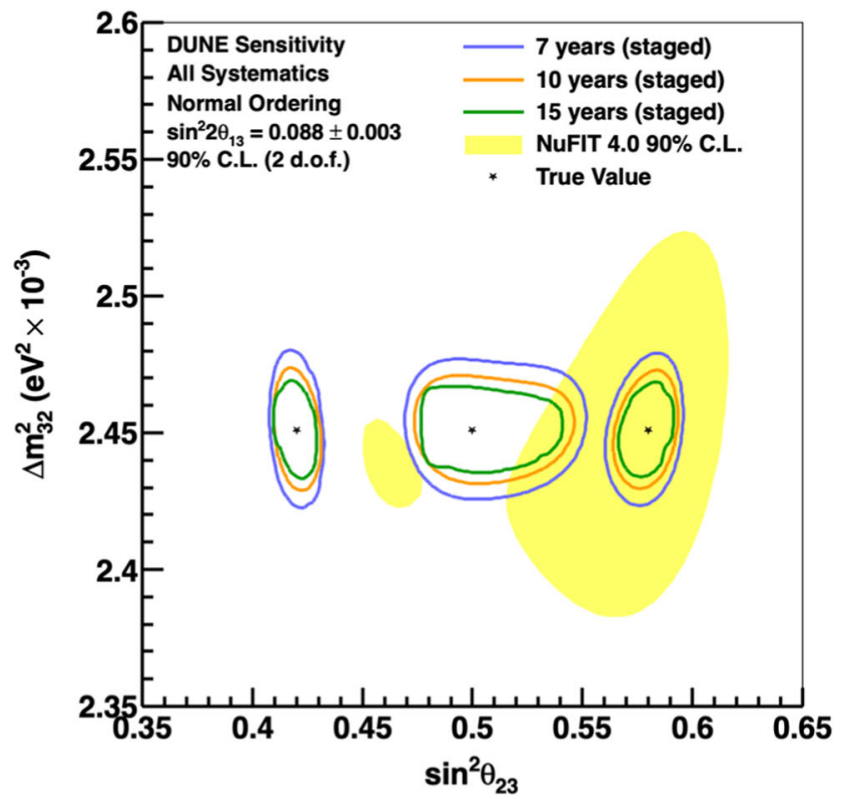

oscillation parameter values used are denoted by stars, and the NuFIT best fit values are used as the true value of all those not explicitly shown. Test values of $\sin ^{2} \theta_{23}=0.42,0.5,0.58$ were used for both top and bottom plots. In the top plot, test values of $\delta_{\mathrm{CP}}=-\pi / 2,0, \pi / 2$ were used

\section{Conclusion}

The analyses presented here are based on full, end-to-end simulation, reconstruction, and event selection of FD Monte Carlo and parameterized analysis of ND Monte Carlo of the DUNE experiment. Detailed uncertainties from flux, the neutrino interaction model, and detector effects have been included in the analysis. Sensitivity results are obtained using a sophisticated, custom fitting framework. These studies demonstrate that DUNE will be able to measure $\delta_{\mathrm{CP}}$ to high precision, unequivocally determine the neutrino mass ordering, and make precise measurements of the parameters governing long-baseline neutrino oscillation.

We note that further improvements are expected once the full potential of the DUNE ND is included in the analysis. In addition to the samples used explicitly in this analysis, the LArTPC is expected to measure numerous exclusive finalstate CC channels, as well as $v_{e}$ and $\mathrm{NC}$ events. Additionally, neutrino-electron elastic scattering [64] and the low- $v$ technique [65-70] may be used to constrain the flux. Additional samples of events from other detectors in the DUNE ND complex are not explicitly included here, but there is an assumption that we will be able to control the uncertainties to the level used in the analysis, and it should be understood that that implicitly relies on having a highly capable ND. 
DUNE will be able to establish the neutrino mass ordering at the $5 \sigma$ level for $100 \%$ of $\delta_{\mathrm{CP}}$ values between two and three years. CP violation can be observed with $5 \sigma$ significance after $\sim 7$ years if $\delta_{\mathrm{CP}}=-\pi / 2$ and after $\sim 10$ years for $50 \%$ of $\delta_{\mathrm{CP}}$ values. $\mathrm{CP}$ violation can be observed with $3 \sigma$ significance for $75 \%$ of $\delta_{\mathrm{CP}}$ values after $\sim 13$ years of running. For 15 years of exposure, $\delta_{\mathrm{CP}}$ resolution between $5^{\circ}$ and $15^{\circ}$ are possible, depending on the true value of $\delta_{\mathrm{CP}}$. The DUNE measurement of $\sin ^{2} 2 \theta_{13}$ approaches the precision of reactor experiments for high exposure, allowing measurements that do not rely on an external $\sin ^{2} 2 \theta_{13}$ constraint and facilitating a comparison between the DUNE and reactor $\sin ^{2} 2 \theta_{13}$ results, which is of interest as a potential signature for physics beyond the standard model. DUNE will have significant sensitivity to the $\theta_{23}$ octant for values of $\sin ^{2} \theta_{23}$ less than about 0.47 and greater than about 0.55 . We note that the results found are broadly consistent with those found in Ref. [7], using a much simpler analysis.

The measurements made by DUNE will make significant contributions to completion of the standard three-flavor mixing picture, and provide invaluable inputs to theory work understanding whether there are new symmetries in the neutrino sector and the relationship between the generational structure of quarks and leptons. The observation of CPV in neutrinos would be an important step in understanding the origin of the baryon asymmetry of the universe. The precise measurements of the three-flavor mixing parameters that DUNE will provide may also yield inconsistencies that point us to physics beyond the standard three-flavor model.

Acknowledgements This document was prepared by the DUNE collaboration using the resources of the Fermi National Accelerator Laboratory (Fermilab), a U.S. Department of Energy, Office of Science, HEP User Facility. Fermilab is managed by Fermi Research Alliance, LLC (FRA), acting under Contract No. DE-AC02-07CH11359. This work was supported by CNPq, FAPERJ, FAPEG and FAPESP, Brazil; CFI, IPP and NSERC, Canada; CERN; MŠMT, Czech Republic; ERDF, H2020-EU and MSCA, European Union; CNRS/IN2P3 and CEA, France; INFN, Italy; FCT, Portugal; NRF, South Korea; CAM, Fundación "La Caixa" and MICINN, Spain; SERI and SNSF, Switzerland; TÜBITAK, Turkey; The Royal Society and UKRI/STFC, UK; DOE and NSF, United States of America. This research used resources of the National Energy Research Scientific Computing Center (NERSC), a U.S. Department of Energy Office of Science User Facility operated under Contract No. DE-AC02-05CH11231.

Data Availability Statement This manuscript has no associated data or the data will not be deposited. [Authors' comment: This manuscript describes a sensitivity study for the DUNE experiment using simulation only, and as such there are no experimental data to report.]

Open Access This article is licensed under a Creative Commons Attribution 4.0 International License, which permits use, sharing, adaptation, distribution and reproduction in any medium or format, as long as you give appropriate credit to the original author(s) and the source, provide a link to the Creative Commons licence, and indicate if changes were made. The images or other third party material in this article are included in the article's Creative Commons licence, unless indi- cated otherwise in a credit line to the material. If material is not included in the article's Creative Commons licence and your intended use is not permitted by statutory regulation or exceeds the permitted use, you will need to obtain permission directly from the copyright holder. To view a copy of this licence, visit http://creativecomm ons.org/licenses/by/4.0/.

Funded by $\mathrm{SCOAP}^{3}$.

\section{References}

1. X. Qian, P. Vogel, Neutrino Mass Hierarchy. Prog. Part. Nucl. Phys. 83, 1-30 (2015). https://doi.org/10.1016/j.ppnp.2015.05. 002. arXiv:1505.01891 [hep-ex]

2. M. Fukugita, T. Yanagida, Baryogenesis Without Grand Unification. Phys. Lett. B 174, 45 (1986). https://doi.org/10.1016/ 0370-2693(86)91126-3

3. S. Davidson, E. Nardi, Y. Nir, Leptogenesis. Phys. Rept. 466, 105-177 (2008). https://doi.org/10.1016/j.physrep.2008.06.002. arXiv:0802.2962 [hep-ph]

4. DUNE Collaboration, B. Abi et al., Deep Underground Neutrino Experiment (DUNE), Far Detector Technical Design Report, Volume I Introduction to DUNE. arXiv:2002.02967 [physics.ins-det]

5. DUNE Collaboration, B. Abi et al., Deep Underground Neutrino Experiment (DUNE), Far Detector Technical Design Report, Volume II DUNE Physics. arXiv:2002.03005 [hep-ex]

6. DUNE Collaboration, R. Acciarri et al., Long-Baseline Neutrino Facility (LBNF) and Deep Underground Neutrino Experiment (DUNE). arXiv:1601.05471 [physics.ins-det]

7. DUNE Collaboration, R. Acciarri et al., Long-baseline neutrino facility (LBNF) and deep underground neutrino experiment (DUNE). arXiv:1512.06148 [physics.ins-det]

8. DUNE Collaboration, R. Acciarri et al., Long-baseline neutrino facility (LBNF) and deep underground neutrino experiment (DUNE). arXiv:1601.02984 [physics.ins-det]

9. I. Esteban, M.C. Gonzalez-Garcia, A. Hernandez-Cabezudo, M. Maltoni, T. Schwetz, Global analysis of three-flavour neutrino oscillations: synergies and tensions in the determination of $\theta_{23}$, $\delta_{C P}$, and the mass ordering. JHEP 01, 106 (2019). https://doi.org/ 10.1007/JHEP01(2019)106. arXiv:1811.05487 [hep-ph]

10. P.F. de Salas, D.V. Forero, C.A. Ternes, M. Tortola, J.W.F. Valle, Status of neutrino oscillations, $3 \sigma$ hint for normal mass ordering and improved CP sensitivity. Phys. Lett. B 782(2018), 633-640 (2018). https://doi.org/10.1016/j.physletb.2018.06.019. arXiv: 1708.01186 [hep-ph]

11. F. Capozzi, E. Lisi, A. Marrone, D. Montanino, A. Palazzo, Status and prospects of global analyses of neutrino mass-mixing parameters. J. Phys: Conf. Ser. 888(1), 012037 (2017). https://doi.org/ 10.1088/1742-6596/888/1/012037

12. T2K Collaboration, K. Abe et al., Search for CP Violation in Neutrino and Antineutrino Oscillations by the T2K Experiment with $2.2 \times 10^{21}$ Protons on Target, Phys. Rev. Lett. 121(17), (2018) 171802, https://doi.org/10.1103/PhysRevLett. 121.171802, arXiv:1807.07891 [hep-ex]

13. Super-Kamiokande Collaboration, K. Abe et al., Atmospheric neutrino oscillation analysis with external constraints in SuperKamiokande I-IV, Phys. Rev. D 97(7), 072001, (2018). https:// doi.org/10.1103/PhysRevD.97.072001, arXiv:1710.09126 [hepex]

14. NOvA Collaboration, M.A. Acero et al., First measurement of neutrino oscillation parameters using neutrinos and antineutrinos by NOvA. Phys. Rev. Lett. 123(15), 151803 (2019). https://doi. org/10.1103/PhysRevLett.123.151803. arXiv:1906.04907 [hepex] 
15. T2K Collaboration, K. Abe et al., Constraint on the matterantimatter symmetry-violating phase in neutrino oscillations. Nature 580(7803), (2020) 339-344, https://doi.org/10.1038/ s41586-020-2177-0, arXiv:1910.03887 [hep-ex]

16. H. Nunokawa, S.J. Parke, J.W. Valle, CP violation and neutrino oscillations. Prog. Part. Nucl. Phys. 60, 338-402 (2008). https:// doi.org/10.1016/j.ppnp.2007.10.001. arXiv:0710.0554 [hep-ph]

17. L. Wolfenstein, Neutrino oscillations in matter. Phys. Rev. D 17, 2369-2374 (1978). https://doi.org/10.1103/PhysRevD.17.2369

18. S. Mikheev, A.Y. Smirnov, Resonance amplification of oscillations in matter and spectroscopy of solar neutrinos. Sov. J. Nucl. Phys. 42, 913-917 (1985)

19. NOvA Collaboration, D. S. Ayres et al., The NOvA technical design report. (2007)

20. T2K Collaboration, K. Abe et al., The T2K Experiment, Nucl. Instrum. Meth. A 659 106-135 (2011). https://doi.org/10.1016/j. nima.2011.06.067, arXiv:1106.1238 [physics.ins-det]

21. Hyper-Kamiokande Collaboration, K. Abe et al., HyperKamiokande Design Report. arXiv:1805.04163 [physics.ins-det]

22. MINERvA Collaboration, L. Aliaga et al., Neutrino Flux Predictions for the NuMI Beam, Phys. Rev. D 94(9), 092005 (2016), arXiv:1607.00704 [hep-ex]. https://doi.org/10. 1103/PhysRevD.94.092005, https://doi.org/10.1103/PhysRevD. 95.039903, [Addendum: Phys. Rev. D95, no.3, 039903 (2017)]

23. GEANT4 Collaboration, S. Agostinelli et al., GEANT4: A Simulation toolkit, Nucl. Instrum. Meth. A 506, 250-303. (2003) https://doi.org/10.1016/S0168-9002(03)01368-8

24. EMPHATIC Collaboration, T. Akaishi et al., EMPHATIC: A proposed experiment to measure hadron scattering and production cross sections for improved neutrino flux predictions. arXiv: 1912.08841 [hep-ex]

25. T. Vladisavljevic, Constraining the T2K Neutrino Flux Prediction with 2009 NA61/SHINE Replica-Target Data, in Proceedings, Prospects in Neutrino Physics (NuPhys2017): London, UK, December 20-22, 2017, pp. 189-193. (2018). https://doi.org/10. 5281/zenodo.1300546, arXiv:1804.00272 [physics.ins-det]

26. DUNE Collaboration, L. Fields, A Flux Spectrometer for LBNF/DUNE, Talk presented at the APS Division of Particles and Fields Meeting (DPF 2017), July 31-August 4 (2017)

27. C. Andreopoulos et al., The GENIE Neutrino Monte Carlo Generator. Nucl. Instrum. Meth. A 614, 87-104 (2010). https://doi. org/10.1016/j.nima.2009.12.009. arXiv:0905.2517 [hep-ph]

28. C. Andreopoulos, C. Barry, S. Dytman, H. Gallagher, T. Golan, R. Hatcher, G. Perdue, J. Yarba, The GENIE neutrino Monte Carlo generator: physics and user manual. arXiv:1510.05494 [hep-ph]

29. P. Stowell et al., NUISANCE: a neutrino cross-section generator tuning and comparison framework. JINST 12(01), P01016 (2017). https://doi.org/10.1088/1748-0221/12/01/P01016. arXiv:1612.07393 [hep-ex]

30. A. Bodek, J. L. Ritchie, Fermi-motion effects in deep-inelastic lepton scattering from nuclear targets. Phys. Rev. D 23, 10701091 (1981) . https://doi.org/10.1103/PhysRevD.23.1070. http:// link.aps.org/doi/10.1103/PhysRevD.23.1070

31. C. Wilkinson et al., Testing charged current quasi-elastic and multinucleon interaction models in the NEUT neutrino interaction generator with published datasets from the MiniBooNE and MINERvA experiments. Phys. Rev. D 93(7), 072010 (2016). https:// doi.org/10.1103/PhysRevD.93.072010. arXiv:1601.05592 [hepex]

32. O. Benhar, A. Fabrocini, S. Fantoni, I. Sick, Spectral function of finite nuclei and scattering of GeV electrons. Nucl. Phys. A 579, 493-517 (1994). https://doi.org/10.1016/0375-9474(94)90920-2

33. J. Nieves, J.E. Amaro, M. Valverde, Inclusive quasi-elastic neutrino reactions. Phys. Rev. C 70, 055503 (2004). arXiv:nucl-th/0408005 [nucl-th]. https://doi.org/10.1103/
PhysRevC.70.055503, https://doi.org/10.1103/PhysRevC.72. 019902, [Erratum: Phys. Rev. C72, 019902(2005)]

34. K. Gallmeister, U. Mosel, J. Weil, Neutrino-induced reactions on nuclei. Phys. Rev. C 94(3), 035502 (2016). https://doi.org/10. 1103/PhysRevC.94.035502. arXiv:1605.09391 [nucl-th]

35. V. Pandey, N. Jachowicz, T. Van Cuyck, J. Ryckebusch, M. Martini, Low-energy excitations and quasielastic contribution to electron-nucleus and neutrino-nucleus scattering in the continuum random-phase approximation. Phys. Rev. C 92(2), 024606 (2015). https://doi.org/10.1103/PhysRevC.92.024606. arXiv:1412.4624 [nucl-th]

36. J.E. Sobczyk, Intercomparison of lepton-nucleus scattering models in the quasielastic region. Phys. Rev. C 96(4), 045501 (2017). https://doi.org/10.1103/PhysRevC.96.045501. arXiv:1706.06739 [nucl-th]

37. J. Nieves, I. Ruiz Simo, M .J. Vicente Vacas, Phys. Rev. C. Inclusive charged-current neutrino-nucleus reactions $\mathbf{8 3}$, 045501 (2011). https://doi.org/10.1103/PhysRevC.83.045501. arXiv:1102.2777 [hep-ph]

38. R. Gran, J. Nieves, F. Sanchez, M .J. Vicente Vacas, Phys. Rev. D. Neutrino-nucleus quasi-elastic and $2 \mathrm{p} 2 \mathrm{~h}$ interactions up to 10 GeV 88(11), 113007 (2013). https://doi.org/10.1103/PhysRevD. 88.113007. arXiv:1307.8105 [hep-ph]

39. M. Valverde, J.E. Amaro, J. Nieves, Theoretical uncertainties on quasielastic charged-current neutrino-nucleus cross sections. Phys. Lett. B 638, 325-332 (2006). https://doi.org/10.1016/j. physletb.2006.05.053. arXiv:hep-ph/0604042 [hep-ph]

40. A.S. Meyer, M. Betancourt, R. Gran, R.J. Hill, Deuterium target data for precision neutrino-nucleus cross sections. Phys. Rev. D 93(11), 113015 (2016). https://doi.org/10.1103/PhysRevD.93. 113015. arXiv: 1603.03048 [hep-ph]

41. R. Bradford, A. Bodek, H. Budd, J. Arrington, A new parameterization of the nucleon elastic form factors. Nucl. Phys. B Proc. Suppl. 159, 127-132 (2006). https://doi.org/10. 1016/j.nuclphysbps.2006.08.028, http://www.sciencedirect.com/ science/article/pii/S0920563206005184

42. J. Schwehr, D. Cherdack, R. Gran, GENIE implementation of IFIC Valencia model for QE-like 2p2h neutrino-nucleus cross section. arXiv:1601.02038 [hep-ph]

43. MINERvA Collaboration, P. A. Rodrigues et al., Identification of nuclear effects in neutrino-carbon interactions at low three-momentum transfer. Phys. Rev. Lett. 116, 071802 (2016). https://doi.org/10.1103/PhysRevLett.116. 071802. arXiv:1511.05944 [hep-ex]

44. NOvA Collaboration, M. A. Acero et al., New constraints on oscillation parameters from $v_{e}$ appearance and $v_{\mu}$ disappearance in the NOvA experiment. Phys. Rev. D 98, 032012 (2018). https:// doi.org/10.1103/PhysRevD.98.032012. arXiv:1806.00096 [hepex]

45. C. Llewellyn Smith, Neutrino reactions at accelerator energies. Phys. Rept. 3, 261-379 (1972). https://doi.org/10.1016/ 0370-1573(72)90010-5

46. C. Colle, O. Hen, W. Cosyn, I. Korover, E. Piasetzky, J. Ryckebusch, L.B. Weinstein, Extracting the mass dependence and quantum numbers of short-range correlated pairs from $A\left(e, e^{\prime} p\right)$ and $A\left(e, e^{\prime} p p\right)$ scattering. Phys. Rev. C $92(2), 024604$ (2015), https:// doi.org/10.1103/PhysRevC.92.024604. arXiv:1503.06050 [nuclth]

47. D. Rein, L.M. Sehgal, Neutrino excitation of baryon resonances and single pion production. Ann. Phys. 133, 79-153 (1981). https://doi.org/10.1016/0003-4916(81)90242-6

48. Particle Data Group Collaboration, M. Tanabashi et al., Review of particle physics. Phys. Rev. D 98(3), 030001 (2018). https:// doi.org/10.1103/PhysRevD.98.030001

49. C. Wilkinson, P. Rodrigues, S. Cartwright, L. Thompson, K. McFarland, Reanalysis of bubble chamber measurements of 
muon-neutrino induced single pion production. Phys. Rev. D 90(11), 112017 (2014). https://doi.org/10.1103/PhysRevD.90. 112017. arXiv:1411.4482 [hep-ex]

50. P. Rodrigues, C. Wilkinson, K. McFarland, Constraining the GENIE model of neutrino-induced single pion production using reanalyzed bubble chamber data. Eur. Phys. J. C 76(8), 474 (2016). https://doi.org/10.1140/epjc/s10052-016-4314-3. arXiv: 1601.01888 [hep-ex]

51. M. Kabirnezhad, Single pion production in neutrino-nucleon interactions, Phys. Rev. D 97, 013002 (2018). https://doi.org/10. 1103/PhysRevD.97.013002

52. A. Bodek, U. Yang, Higher twist, xi(omega) scaling, and effective LO PDFs for lepton scattering in the few GeV region. J. Phys. G 29, 1899-1906 (2003). https://doi.org/10.1088/0954-3899/29/8/ 369. arXiv:hep-ex/0210024

53. M. Glück, E. Reya, A. Vogt, Dynamical parton distributions revisited. Eur. Phys. J. C 5, 461-470 (1998). https://doi.org/10.1007/ s100520050289. arXiv:hep-ph/9806404

54. T. Yang, C. Andreopoulos, H. Gallagher, K. Hoffmann, P. Kehayias, A hadronization model for few-GeV neutrino interactions. Eur. Phys. J. C 63, 1-10 (2009). https://doi.org/10.1140/ epjc/s10052-009-1094-z. arXiv:0904.4043 [hep-ph]

55. Z. Koba, H.B. Nielsen, P. Olesen, Scaling of multiplicity distributions in high-energy hadron collisions. Nucl. Phys. B 40, 317-334 (1972). https://doi.org/10.1016/0550-3213(72)90551-2

56. T. Sjostrand, S. Mrenna, P.Z. Skands, PYTHIA 6.4 physics and manual. JHEP 05, 026 (2006). https://doi.org/10.1088/ 1126-6708/2006/05/026. arXiv:hep-ph/0603175 [hep-ph]

57. M. Sanchez, Nova results and prospects, in XXVIII International Conference on Neutrino Physics and Astrophysics (Neutrino 2018). Zenodo, June, (2018). https://doi.org/10.5281/zenodo. 1286758, https://zenodo.org/record/1286758

58. S. Dytman, A. Meyer, Final state interactions in GENIE. AIP Conf. Proc. 1405, 213-218 (2011). https://doi.org/10.1063/1. 3661588

59. S. Dytman, GENIE final state interactions. AIP Conf. Proc. 1680, 020005 (2015). https://doi.org/10.1063/1.4931864

60. S. Dytman, Final state interactions in neutrino-nucleus experiments. Acta Phys. Polon. B 40, 2445-2460 (2009)

61. E.S. Pinzon Guerra et al., Using world charged $\pi^{ \pm}$-nucleus scattering data to constrain an intranuclear cascade model. Phys. Rev. D 99(5), 052007 (2019). https://doi.org/10.1103/PhysRevD.99. 052007. arXiv:1812.06912 [hep-ex]

62. M. Day, K.S. McFarland, Differences in quasi-elastic crosssections of muon and electron neutrinos. Phys. Rev. D 86, 053003 (2012). https://doi.org/10.1103/PhysRevD.86.053003. arXiv: 1206.6745 [hep-ph]

63. nuPRISM Collaboration, S. Bhadra et al., Letter of intent to construct a nuPRISM detector in the J-PARC neutrino beamline. arXiv:1412.3086 [physics.ins-det]

64. C.M. Marshall, K.S. McFarland, C. Wilkinson, Neutrino-electron elastic scattering for flux determination at the DUNE oscillation experiment. Phys. Rev. D 101(3), 032002 (2020). https://doi.org/ 10.1103/PhysRevD.101.032002. arXiv:1910.10996 [hep-ex]

65. P.Z. Quintas et al., A Measurement of $\Lambda_{\overline{M S}}$ from $v_{\mu}$ - Fe Nonsinglet Structure Functions at the Fermilab Tevatron. Phys. Rev. Lett. 71, 1307-1310 (1993). https://doi.org/10.1103/PhysRevLett.71. 1307

66. CCFR/NuTeV Collaboration, U.-K. Yang et al., Measurements of $F_{2}$ and $x F_{3}^{v}-x F_{3}^{\bar{v}}$ from CCFR $v_{\mu}-\mathrm{Fe}$ and $\bar{v}_{\mu}-\mathrm{Fe}$ data in a physics model independent way, Phys. Rev. Lett. 86, 2742-2745, (2001). https://doi.org/10.1103/PhysRevLett.86. 2742. arXiv:hep-ex/0009041 [hep-ex]

67. NuTeV Collaboration, M. Tzanov et al., Precise measurement of neutrino and anti-neutrino differential cross sections. Phys.
Rev. D 74, 012008 (2006). https://doi.org/10.1103/PhysRevD.74. 012008, arXiv:hep-ex/0509010 [hep-ex]

68. MINOS Collaboration, P. Adamson et al., Neutrino and antineutrino inclusive charged-current cross section measurements with the MINOS near detector. Phys. Rev. D 81, 072002 (2010). https://doi.org/10.1103/PhysRevD.81.072002. arXiv:0910.2201 [hep-ex]

69. MINERvA Collaboration, J. Devan et al., "Measurements of the inclusive neutrino and antineutrino charged current cross sections in MINERvA using the low-v flux method. Phys. Rev. D 94(11), 112007 (2016). https://doi.org/10.1103/PhysRevD.94. 112007. arXiv:1610.04746 [hep-ex]

70. MINERvA Collaboration, L. Ren et al., Measurement of the antineutrino to neutrino charged-current interaction cross section ratio in MINERvA. Phys. Rev. D 95(7), 072009 (2017). arXiv:1701.04857 [hep-ex]. https://doi.org/10.1103/ PhysRevD.97.019902. https://doi.org/10.1103/PhysRevD.95. 072009 [Addendum: Phys. Rev. D97, no.1, 019902(2018)]

71. MicroBooNE Collaboration, P. Abratenko et al., Determination of muon momentum in the MicroBooNE LArTPC using an improved model of multiple Coulomb scattering. JINST 12(10), P10010 (2017). https://doi.org/10.1088/1748-0221/12/ 10/P10010. arXiv:1703.06187 [physics.ins-det]

72. ArgonCube Collaboration, C. Amsler et al., ArgonCube: a novel, fully-modular approach for the realization of large-mass liquid argon TPC neutrino detectors, Tech. Rep. CERN-SPSC-2015009. SPSC-I-243, CERN, Geneva (2015). https://cds.cern.ch/ record/1993255

73. D.A. Dwyer et al., LArPix: demonstration of low-power 3D pixelated charge readout for liquid argon time projection chambers. JINST 13(10), P10007 (2018). https://doi.org/10.1088/ 1748-0221/13/10/P10007. arXiv:1808.02969 [physics.ins-det]

74. M. Auger, Y. Chen, A. Ereditato, D. Goeldi, I. Kreslo, D. Lorca, M. Luethi, T. Mettler, J.R. Sinclair, M.S. Weber, ArCLight-a compact dielectric large-area photon detector. Instruments 2(1), 3 (2018). https://doi.org/10.3390/instruments2010003. arXiv:1711.11409 [physics.ins-det]

75. L. Emberger, F. Simon, A highly granular calorimeter concept for long baseline near detectors. J. Phys: Conf. Ser. 1162(1), 012033 (2019). https://doi.org/10.1088/1742-6596/ 1162/1/012033. arXiv:1810.03677 [physics.ins-det]

76. DUNE Collaboration, B. Abi et al., The DUNE far detector interim design report volume 1: physics, technology and strategies. arXiv:1807.10334 [physics.ins-det]

77. DUNE Collaboration, B. Abi et al., The DUNE far detector interim design report, Volume 2: single-phase module. arXiv:1807.10327 [physics.ins-det]

78. DUNE Collaboration, B. Abi et al., The DUNE far detector interim design report, Volume 3: dual-phase module. arXiv: 1807.10340 [physics.ins-det]

79. DUNE Collaboration, B. Abi et al., Deep underground neutrino experiment (DUNE), far detector technical design report, Volume IV far detector single-phase technology. arXiv:2002.03010 [physics.ins-det]

80. J.S. Marshall, M.A. Thomson, The Pandora software development kit for pattern recognition. Eur. Phys. J. C 75(9), 439 (2015). https://doi.org/10.1140/epjc/s10052-015-3659-3. arXiv: 1506.05348 [physics.data-an]

81. MicroBooNE Collaboration, R. Acciarri et al., The Pandora multialgorithm approach to automated pattern recognition of cosmicray muon and neutrino events in the MicroBooNE detector, Eur. Phys. J. C 78(1), 82 (2018). https://doi.org/10.1140/epjc/ s10052-017-5481-6, arXiv:1708.03135 [hep-ex]

82. DUNE Collaboration, B. Abi et al., Neutrino interaction classification with a convolutional neural network in the DUNE far detector. arXiv:2006.15052 [physics.ins-det] 
83. I. Esteban, M.C. Gonzalez-Garcia, A. Hernandez-Cabezudo, M. Maltoni, T. Schwetz, "Nufit4.0," 2018. http://www.nu-fit.org/

84. B. Roe, Matter density versus distance for the neutrino beam from Fermilab to Lead, South Dakota, and comparison of oscillations with variable and constant density. Phys. Rev. D 95(11), 113004 (2017). https://doi.org/10.1103/PhysRevD.95. 113004. arXiv:1707.02322 [hep-ex]

85. MicroBooNE Collaboration, C. Adams et al., A method to determine the electric field of liquid argon time projection chambers using a uv laser system and its application in microboone. arXiv: 1910.01430 [physics.ins-det]

86. CAPTAIN Collaboration, H. Berns et al., The CAPTAIN detector and physics program. arXiv:1309.1740 [physics.ins-det]

87. CAPTAIN Collaboration, B. Bhandari et al., First measurement of the total neutron cross section on argon between 100 and 800 MeV. arXiv: 1903.05276 [hep-ex]

88. ArgoNeuT Collaboration, R. Acciarri et al., Demonstration of $\mathrm{MeV}$-scale physics in liquid argon time projection chambers using ArgoNeuT. Phys. Rev. D 99(1), 012002 (2019). https://doi.org/10. 1103/PhysRevD.99.012002arXiv:1810.06502 [hep-ex]

89. MINERvA Collaboration, M. Elkins et al., Neutron measurements from anti-neutrino hydrocarbon reactions. arXiv:1901.04892 [hep-ex]

90. MicroBooNE Collaboration, C. Adams et al., First Measurement of $v_{\mu}$ Charged-Current $\pi^{0}$ Production on Argon with a LArTPC, arXiv: 1811.02700 [hep-ex]

91. DUNE Collaboration, T. Alion et al., Experiment simulation configurations used in DUNE CDR, arXiv:1606.09550 [physics.insdet]

92. M. Bass, Neutrino oscillation parameter sensitivity in future longbaseline experiments. PhD thesis, Colorado State U., (2014). https://doi.org/10.2172/1172561

93. NOvA Collaboration, ed., NOvA-ART, ch. CAFAna overview. Redmine, (2019). https://cdcvs.fnal.gov/redmine/projects/ novaart/wiki/CAFAna_overview
94. F. James, MINUIT function minimization and error analysis: reference manual Version 94.1. CERN-D-506, CERN-D506, (1994)

95. Double Chooz Collaboration Collaboration, Y. Abe et al., Improved measurements of the neutrino mixing angle $\theta_{13}$ with the Double Chooz detector, JHEP10 086, (2014), arXiv:1406.7763 [hep-ex]. https://doi.org/10.1007/JHEP02(2015)074. https://doi. org/10.1007/JHEP10(2014)086 [Erratum: JHEP02,074(2015)]

96. Daya Bay Collaboration, D. Adey, et al., Measurement of electron antineutrino oscillation with, days of operation at Daya Bay. Phys. Rev. Lett. 121(2018), 241805 (1958). arXiv:1809.02261 [hep-ex]

97. RENO Collaboration, G. Bak et al., Measurement of reactor antineutrino oscillation amplitude and frequency at RENO. Phys. Rev. Lett. 121, 201801 (2018). arXiv: 1806.00248 [hep-ex]

98. G. Cowan, K. Cranmer, E. Gross, O. Vitells, Asymptotic formulae for likelihood-based tests of new physics. Eur. Phys. J. C 71, 1554 (2011). arXiv:1007.1727 [physics.data-an]. https://doi.org/ 10.1140/epjc/s10052-011-1554-0, https://doi.org/10.1140/epjc/ s10052-013-2501-z, [Erratum: Eur. Phys. J. C73, 2501(2013)]

99. E. Ciuffoli, J. Evslin, X. Zhang, Confidence in a neutrino mass hierarchy determination. JHEP 01, 095 (2014). https://doi.org/10. 1007/JHEP01(2014)095. arXiv:1305.5150 [hep-ph]

100. X. Qian, A. Tan, W. Wang, J. Ling, R. McKeown et al., Statistical evaluation of experimental determinations of neutrino mass hierarchy. Phys. Rev. D 86, 113011 (2012). https://doi.org/10.1103/ PhysRevD.86.113011. arXiv:1210.3651 [hep-ph]

101. M. Blennow, P. Coloma, P. Huber, T. Schwetz, Quantifying the sensitivity of oscillation experiments to the neutrino mass ordering. JHEP 1403, 028 (2014). https://doi.org/10.1007/ JHEP03(2014)028. arXiv:1311.1822 [hep-ph] 